\title{
Effects of q(r) on the Alpha Particle Ripple Loss in TFTR
}

\author{
S.J. Zweben, D.S. Darrow, S. Batha ${ }^{\dagger}$, R.V. Budny, M. Diesso, \\ H. W. Herrmann, J. Giarrusso, M. Redi, H. Takahashi, S. von Goeler, \\ R.B. White, and R. Wieland \\ Princeton Plasma Physics Laboratory \\ P.O. Box 451, Princeton, N.J. 08543 \\ $\dagger$ Fusion Physics and Technology, Inc., Torrance, Calif. 90503
}

\begin{abstract}
An experiment was done with TFTR DT plasmas to determine the effect of the $q(r)$ profile on the alpha particle ripple loss to the outer midplane. The alpha particle loss measurements were made using a radially movable scintillator detector $20^{\circ}$ below the outer midplane. The experimental results were compared with TF ripple loss calculations done using a Monte Carlo guiding center orbit following code, ORBIT. Although some of the experimental results are consistent with the ORBIT code modeling, the variation of the alpha loss with the $q(r)$ profiles is not well explained by this code. Quantitative interpretation of these measurements requires a careful analysis of the limiter shadowing effect, which strongly determines the diffusion of alphas into the detector aperture.
\end{abstract}




\section{Introduction}

The goal of the present experiment was to test the predicted $q(\mathrm{r})$ dependence of the standard theory for toroidal field (TF) ripple-induced "stochastic ripple diffusion" (SRD) using DT alpha particles in TFTR. According to the original SRD model [1], trapped alpha particles will randomly and rapidly diffuse radially when their banana tips fall in a region where the $\mathrm{TF}$ ripple is larger than:

$$
\delta_{\mathrm{GWB}} \approx(\varepsilon / N \pi q)^{3 / 2}\left(1 / \rho q^{\prime}\right)
$$

Here $\delta_{\mathrm{GWB}}$ is the Goldston-White-Boozer stochastic threshold ( $\delta$ is the peakto-average $\delta \mathrm{B}_{\text {tor }} / \mathrm{B}_{\text {tor }}$ along a field line), $\varepsilon=\mathrm{r} / \mathrm{R}$ is the location of the ion's banana tip (where the orbit is most sensitive to the TF ripple perturbation), $\mathrm{N}$ is the number of TF coils, $\rho$ is the orbit's gyroradius, and $q$ and $q^{\prime}(=\partial q / \partial \rho)$ depend on the magnetic $q(r)$ profile at the banana tip.

When this stochasticity condition is satisfied, the variations of the alpha banana tip location due to the non-axisymmetric TF ripple become decorrelated between successive bounces, causing the orbit to diffuse radially until it leaves the stochastic region (usually by being lost to the wall). Note that this type of banana tip diffusion is fundamentally different from the ripple loss which occurs when an ion is trapped in the low field region between two $\mathrm{TF}$ coils. The latter occurs only for a small range of pitch angles nearly perpendicular to the toroidal field, whereas SRD occurs for any trapped ions over a wide range of pitch angles.

Although there were several simplifying assumptions used in deriving Eq. 1, a fundamental feature of this model is that the SRD threshold should depend on the $q(r)$ profile, which affects the stochasticity condition of the banana orbit tip. More recent theory and modeling have generalized the result of Eq. 1 to include non-circular equilibria, but the sensitivity of the SRD mechanism to the $q(r)$ profile remains [2]. This sensitivity to $q(r)$ may be important for future tokamak reactors in which the alpha loss due to $\mathrm{TF}$ ripple is predicted to be unusually high in the "advanced" tokamak regime at high $q(0)$ [3]. 
Several experimental studies have been made of the effects of $\mathrm{TF}$ ripple on fast ion confinement in tokamaks [4], mainly focusing on ripple trapping and not SRD. Recently measurements were made of outer first wall heating due to ripple trapping and loss of $90 \mathrm{keV} \mathrm{D}$ beam ions in JT$60 \mathrm{U}$ [5], and good agreement was found between the heating pattern and TF ripple loss calculations. Ripple transport experiments have also been performed in JET [6] using $1 \mathrm{MeV}$ triton burnup, in Tore Supra [7] using a calorimeter detector to measure the loss of $200 \mathrm{keV}$ minority ICRH tail ions, and in TFTR [8] using the pellet charge exchange analyzer (PCX). Although these experiments showed generally good agreement with the modeling of TF ripple diffusion and loss, none of them tested the $q(r)$ profile dependence of the SRD mechanism. The first experiment which identified a $q(r)$ dependence of ripple loss was done recently using triton burnup on JT-60U [9], but direct measurements of loss to the wall were not made.

The experiment described in this paper uses the midplane fast ion scintillator detector previously used for measuring the ripple loss of DD fusion products in TFTR [10,11]. Those results showed several of the characteristics expected for TF ripple loss; in particular, the measured pitch angle distribution peaked near the expected angle for TF ripple loss (significantly higher than expected for first-orbit loss), and the total fusion product loss fraction (per DD neutron) varied with plasma current similarly to predictions from a collisionless MAPLOS (bounce-mapping) code [10]. The radial diffusion of DD fusion products was also measured in the shadow of both the outer limiter and a second probe, and the inferred radial step sizes were similar to those predicted by the collisionless GWB model [11].

An overview of the DT alpha loss as measured by this midplane detector showed the following trends [12]: (a) for a given type of plasma, the DT and DD fusion product loss fractions (per neutron) were similar, as expected from the SRD model since their gyro-radii are similar (Eq. 1), (b) the DT alpha loss per neutron peaked at a plasma current of I=1.0 MA, not quite as expected from the collisionless MAPLOS TF ripple loss calculations, (c) at plasma currents of $\mathrm{I}=1-2 \mathrm{MA}$, the pitch angle of the measured loss was roughly consistent with the MAPLOS calculation of TF 
ripple loss, but this was measured only $1.7 \mathrm{~cm}$ behind the limiter shadow, and (d) the alpha loss signal at I=2.0 MA varied by a factor of 20 when the detector aperture was moved $\pm 1.5 \mathrm{~cm}$ around the shadow of the outer limiter, and the pitch angle distribution also varied considerably over this range.

The present paper describes an experiment to determine the $q(\mathrm{r})$ dependence of the alpha particle ripple loss in TFTR, and an attempt to understand this data using the ORBIT guiding center code (i.e. not simply using Eq. 1). The conclusion from this attempt is that the experimental results can not be understood using these ORBIT calculations alone, since they do not correctly take into account the influence of the outer limiters on the alpha particle collection by this detector. This "limiter shadowing" effect strongly determines the radial profile of the detected alpha loss signal near the outer midplane wall, since the alpha orbits must make a radial jump behind this limiter shadow in order to be detected. A preliminary limiter shadowing model is described which can qualitatively explain some of the experimental results, but uncertainties in the vacuum fields and the alpha energy spectrum prevent a quantitative understanding of the data.

The experimental design is described in Sec. II, the detector and limiter geometry is described in Sec. III, and the ORBIT code modeling of $\mathrm{TF}$ ripple loss is described in Sec. IV. The experimental results and comparisons with the ORBIT code are in Sec. V, a description of the limiter shadowing effect is in Sec. VI, a discussion is in Sec. VII, and the Conclusions are in Sec. VIII.

\section{Experimental Design}

This experiment aimed to measure the $q(r)$ dependence of the stochastic TF ripple diffusion (SRD) using DT alpha particles. The experiment was designed to vary the quantity $\left(q^{3 / 2} q^{\prime} \rho\right)$ in Eq. 1 which determines the location of the SRD threshold within the TF ripple of TFTR. The actual experiment was affected by some additional constraints: 
1) The DT reaction rate needed to be much larger than the $\mathrm{DD}$ rate to insure that the midplane probe was detecting primarily alphas, but as small as possible to conserve tritium. Therefore only one tritium-fueled NBI source was used, which produced $\approx 10^{17}$ DT neutrons/sec (i.e. about 50 times the $\mathrm{DD}$ reaction rate).

2) The plasma profiles needed to be kept as constant as possible to help isolate the $q(r)$ profile effects, and free of MHD activity which could cause additional alpha loss. Therefore all of these discharges used a low total NBI power of 7.5 MW ( $1 \mathrm{~T}$ beam and $2 \mathrm{D}$ beams) which are far from the MHD beta limit,

3) Although the TF ripple level is maximized for plasmas which touch the outer limiter boundary (i.e. with $\mathrm{R} \approx 2.62 \mathrm{~m}$ ), this experiment used smaller plasmas with $\mathrm{R}=2.52 \mathrm{~m}$ to allow the detector to be positioned inside the limiter radius as part of the three-point radial scan made for each plasma condition. Similar $\mathrm{R}=2.52 \mathrm{~m}$ plasmas were used to study the firstorbit alpha loss to the bottom detectors [13]. One detector scan at $\mathrm{R}=2.45 \mathrm{~m}$ was added for comparison purposes.

Within these constraints, two plasma scans were done to vary the $q(r)$ profile as widely as possible, and one was done to vary the alpha gyroradius $\rho$ at fixed $q(a)$. All three scans had a common "baseline" plasma of $\mathrm{I}=1.4$ $\mathrm{MA}, \mathrm{B}=4.7 \mathrm{~T}, \mathrm{R}=2.52 \mathrm{~m}, \mathrm{a}=0.87 \mathrm{~m}$, as shown by the "straight-across" case in Fig. 1, with 7.5 MW of NBI from 4.0 to 4.8 sec. More specifically, these scans were:

a) a "q(r)" scan done by initially ramping-up or down the plasma current while keeping it constant at $\mathrm{I}=1.4 \mathrm{MA}$ and $\mathrm{B}=4.7 \mathrm{~T}$ during $\mathrm{NBI}$, as illustrated in Fig. 1. The current ramp-down from I=2.2 MA produced a relatively high current density and low $q(r)$ in the plasma core, while the ramp-up from $\mathrm{I}=0.6 \mathrm{MA}$ produced a low current density and high $\mathrm{q}(\mathrm{r})$ in the core. A higher $q(r)$ should decrease the SRD threshold and increase the ripple loss [3], assuming a similar q'(r) profile in the core. 
b) A "q(a) scan" at a constant I=1.4 MA with toroidal fields of $\mathrm{B}=4.7$, 3.9, and 3.4 T, similar to the "straight-across" case shown in Fig. 1. This scan decreases both $q$ and $q$ ' with decreasing $B$, which should increase the stochastic ripple threshold and decrease the ripple loss, according to Eq. 1, The increased $\rho$ with decreasing $B$ only partially offsets the expected increase in the SRD threshold.

c) A " $\rho$ scan" at fixed $q(a)$ with toroidal fields of $\mathrm{B}=4.7,3.9$, and $3.4 \mathrm{~T}$ at plasma currents of $\mathrm{I}=1.4,1.15$ and 1.0 MA, respectively. This scan varied the alpha gyroradius $\rho$ at a nearly fixed $q(r)$. The stochastic ripple threshold should decrease and the ripple loss increase with decreasing $\mathrm{B}$, according to Eq. 1.

The plasma conditions for these discharges are shown in Table 1 . The central plasma parameters were nearly constant for all cases, e.g. $\mathrm{n}_{\mathrm{e}}(0)=3.0 \pm 0.5 \times 10^{13} \mathrm{~cm}^{-3}$ and $\mathrm{T}_{\mathrm{e}}(0)=5.5 \pm 0.5 \mathrm{keV}$ (both averaged over 4.5-4.8 sec). There was little coherent MHD activity in these discharges, although the discharges with low $q(a)$ did have sawteeth, but these did not significantly affect the time-averaged alpha loss rate.

The actual $q(r)$ profiles for typical plasmas in these scans are shown in Fig. 2, based on motional Stark effect (MSE) measurements of the pitch angle of the magnetic field [14]. The $q(r)$ profiles varied about as expected. The profiles in Fig. 2 were taken $0.1 \mathrm{sec}$ after the start of NBI $(4.6 \mathrm{sec})$, but did not change during the period analyzed for alpha ripple loss $(4.5-4.8 \mathrm{sec})$. Analytic fits of $q(r)$ used in the ORBIT modeling are given in Table 2, along with the measured central Shafranov shifts (see Sec. IV). The vacuum magnetic fields were not directly measured (see Appendix 1).

The SRD regions for these discharges also depend on the TF ripple strength, which is given by a fit to the TFTR magnetic design specification:

$$
\delta(\text { peak-to-average })=6 \times 10^{-6} \exp \left\{\left[(\mathrm{R}-2.25)^{2}+1.31 \mathrm{z}^{2}\right]^{1 / 2} / 0.166\right\}
$$

where $\mathrm{R}$ is the major radius (in $\mathrm{m}$ ) and $\mathrm{z}$ is the height above the midplane (TFTR is symmetrical about the midplane). This is a slightly improved fit to 
that used previously [10]. The TF ripple for these $\mathrm{R}=2.52 \mathrm{~m}$ discharges is therefore $\delta \approx 0.5 \%$ at the outer edge of the plasma at the midplane $(\mathrm{z}=0)$, $\delta \approx 2 \%$ at the outer limiter at the midplane, and $\delta \approx 0.3 \%$ at the top edge of the plasma $(\mathrm{R}=2.52, \mathrm{z}=0.87)$.

A sample evaluation of the stochastic criterion (Eq. 1) for $3.5 \mathrm{MeV}$ alphas is given in Fig. 3, along with the TF ripple strength evaluated vs. $\mathrm{z}$ (vertically at the major radius) and $\mathrm{R}$ (horizontally along at $\mathrm{z}=0$ ). The $\mathrm{SRD}$ threshold location for the baseline "high $q(a)$ " case occurs at $r / a \approx 0.35$, and moves radially outward with decreasing $B$ by $\approx 10-15 \mathrm{~cm}$ as $\left(q^{3 / 2} q^{\prime} \rho\right)$ decrease by a factor of $\approx 3$ for the lower $q(a)$ cases. The relatively small change in the SRD boundary is due to the exponential radial variation of the $\mathrm{TF}$ ripple strength. The actual modeling of the ripple loss in this experiment is done using the Monte Carlo ORBIT guiding center code [15], which takes into account the alpha birth profile, Shafranov shift, and collisionality which are not included in the simple SRD criterion of Eq. 1.

\section{Detector and Limiter Geometry}

The alpha diagnostic for this experiment was a radially movable "midplane" scintillator detector located about $20^{\circ}$ below the outer midplane (in the ion grad-B drift direction), near where the TF ripple loss was expected [10-12]. This detector has been extensively described elsewhere $[16,17]$. The alphas enter the detector aperture through a small "pinhole" $(0.07 \mathrm{~cm} \times 0.2 \mathrm{~cm})$, pass through a wider "slit" $(.07 \mathrm{~cm} \times 1.35 \mathrm{~cm})$, and strike a scintillator screen located inside a light-tight enclosure, as illustrated in Fig. 4.

This aperture pair separates alphas with respect to their pitch angle $X$ (with $X=90^{\circ}$ perpendicular to the toroidal field $\mathrm{B}$ ) and their gyroradius $\rho=(2 \mathrm{ME})^{0.5 / q B}$. Note that this gyroradius assumes $\chi=90^{\circ}$, i.e. $\rho$ is a measure of the alpha energy. The 2-D scintillator light images are sent through an in-vessel coherent quartz fiberoptic bundle (20x20 fibers) to an ex-vessel coherent quartz bundle (50x50 fibers), and are digitized by an intensified CCD camera system every $33 \mathrm{~ms}[12,13]$. Thus the measured quantities are 
the alpha particle flux vs. time through the aperture as a function of the local pitch angle (which measures the magnetic moment) and the local gyroradius (which measures the alpha energy). The total light from the fiberoptic bundle is also measured by a photomultiplier tube for a faster time response.

The alpha gyroradius range covered by this detector is $\rho \approx 2-12 \mathrm{~cm}$, which includes the $\rho \approx 8-11 \mathrm{~cm}$ expected for $3.5 \mathrm{MeV}$ alphas at the detector. The range of pitch angles covered by this detector is $X \approx 40-85^{\circ}$ with respect to the local toroidal $\mathrm{B}$, which includes the whole range of expected SRD loss, but does not include alphas which are trapped in the local ripple wells at angles near $90^{\circ}$ (the expected ripple-trapped alpha fraction is small, as described in Sec. IV).

The pitch and gyroradius measurements are made by interpreting the 2-D scintillator light emission pattern in terms of a coordinate system derived from a detector simulation code, as shown at the bottom of Fig. 4. Each grid point on this coordinate system represents the centroid of the impact locations of an ensemble of alpha orbits entering the aperture pair at a given $\chi$ and $\rho$. The widths of these distributions are due to the finite size of the apertures. The detector design was optimized for good pitch angle resolution $\left(\approx 6^{\circ} \mathrm{FWHM}\right)$ and not for good gyroradius (i.e. energy) resolution, since the energy distribution broadened largely by the beamtarget Doppler shift of $\approx 0.5 \mathrm{MeV}$ FWHM. The pitch and gyroradius have a systematic uncertainty of about $\pm 3^{\circ}$ and $\pm 1 \mathrm{~cm}$, respectively [12], which corresponds to a position uncertainty of \pm 1 pixel or line in Fig. 4 , or $\approx 0.1 \mathrm{~cm}$ on the surface of the $2.5 \mathrm{~cm} \times 2.5 \mathrm{~cm}$ square scintillator.

An important and difficult feature of the present experiment is the effect of the limiter geometry, since most of the alphas must diffuse into the geometrical projection or "shadow" of the outer midplane limiters in order to enter the detector aperture $[11,12,18]$. This limiter geometry is shown schematically in Fig. 5. The most important limiters for this experiment are the "RF limiters" which cover $\pm 30^{\circ}$ around the outer midplane at toroidal locations $\approx 100^{\circ}-170^{\circ}$ from the alpha detector. The nearest limiter is only $45^{\circ}$ away toroidally but does not extend within $\pm 30^{\circ}$ of the outer 
midplane. All limiters are circular with major and minor radii of $\mathrm{R}_{\lim }=260.6 \mathrm{~cm}$ and alim=99 $\mathrm{cm}$, which define the "geometrical shadow" of this limiter set. The limiter alignment uncertainty is about $\pm 0.3 \mathrm{~cm}$, and each has a toroidal extent of $\approx 50 \mathrm{~cm}$. The first wall is much farther outward at $\mathrm{a}_{\mathrm{wall}} \approx 110 \mathrm{~cm}$.

The detector aperture was moved horizontally between $r_{a p}=-2.0 \mathrm{~cm}$ and $r_{a p}=+1.0 \mathrm{~cm}$ with respect to the geometrical shadow of these limiters, which is defined here as $r_{a p}=0 \mathrm{~cm}$ (i.e. when the pinhole aperture is at $\mathrm{R}=352.5 \mathrm{~cm}$ and $\mathrm{z}=-35.6 \mathrm{~cm}$ ). Although for $\mathrm{r}_{\mathrm{ap}}=-2.0 \mathrm{~cm}$ the aperture itself is $2 \mathrm{~cm}$ radially inside the geometrical limiter shadow, alpha orbits entering the aperture at this position could have been behind the limiter shadow when they were nearer to the outer midplane. The limiter shadowing effect is significant in determining the alpha loss to the detector for most of the aperture positions in this experiment (see Sec. VI and the Appendix 1).

\section{ORBIT Code Modeling of TF Ripple Loss}

The experimental results in this paper are mainly compared with modeling done using the ORBIT guiding center code, another version of which was previously used to model TFTR alpha ripple loss $[2,15,19]$. This code calculates alpha particle orbit trajectories in the presence of TF ripple and collisions, and determines the characteristics of the alpha particle loss to the wall, e.g. its pitch angle, energy, and poloidal angle distributions.

The advantage of this code is that it contains all the physics necessary to accurately describe the stochastic ripple diffusion of alpha orbits in TFTR. The main disadvantage of this code is that it assumes that the wall is a smooth toroidal surface, so does not correctly calculate the effect of the limiter shadowing on the local alpha flux to the detector. The strategy of the analysis in this paper is to first compare the experimental results with the ORBIT code modeling (Sec. V), and then (since good agreement is not found) to explore the modifications caused by the limiter shadowing effect (Sec. VI). 
The inputs to the ORBIT code were fits to the $q(r)$ profiles provided by the MSE/VMEC equilibrium analysis [14], and the Abel-inverted neutron (i.e. alpha) source profile shapes measured by the multichannel neutron collimator [20]. These fits are listed in Table 2. To obtain a magnetic equilibrium, the code also inputs the plasma boundary and magnetic axis locations, the shape of the plasma pressure profile, and the toroidal magnetic field on the magnetic axis.

The ORBIT equilibrium flux surfaces as calculated for the baseline $\mathrm{I}=1.4 \mathrm{MA}, \mathrm{B}=4.7 \mathrm{~T}$ case are shown in Fig. 6 . The plasma edge nearly touches the inner limiter at the midplane, but there is a vacuum region outside the plasma but inside the limiter shadow between $\mathrm{R}=3.39 \mathrm{~m}$ and 3.60 $\mathrm{m}$ at the outboard midplane where the magnetic fields were not measured. The flux surfaces in these equilibria are assumed to be circular, and the vacuum field is fit by a smooth curve between the plasma edge and the outer midplane limiter. The vacuum fields at the outer midplane limiter were initially assumed to have $\mathrm{q}=10$ and a Shafranov shift of $-5 \mathrm{~cm}$, but later analysis (see Appendix 1) showed a $\mathrm{q}=25$ and shift of $-17 \mathrm{~cm}$ at the outer limiter (the fields at the plasma edge were the same). The global ORBIT ripple loss modeling was not sensitive to the assumed vacuum fields, since it depended mainly on the banana tip locations inside the plasma, but the limiter shadowing was very sensitive to the assumed vacuum fields (see Sec. VI). The regions of localized magnetic wells due to TF ripple are also shown in Fig. 6, inside of which alpha banana tips can be trapped and lost between TF coils (this is highly unlikely in TFTR).

A single TF ripple loss orbit calculated for the baseline case using this code is shown in Fig. 7. The banana tip was located at a relatively large r/a so that successive poloidal transits had a random vertical displacement of the banana tip characteristic of SRD. The numerical accuracy of this code is such that when the TF ripple and collisionality are set to zero, orbits such as that in Fig. 7 circulate for $>50,000$ transits with a radial motion of $<0.1 \mathrm{~cm}$.

An important factor in the $\mathrm{TF}$ ripple loss modeling is Coulomb collisions, which cause alpha banana orbits to change their magnetic 
moment and/or move from confined to unconfined regions. Previous numerical modeling of TFTR has shown that collisions can increase the total alpha ripple loss by about a factor of two [15,19]. The main factor determining the collisional effect is the ratio of the alpha particle energy efolding rate $\nu_{\alpha, E}$ to the pitch angle scattering rate $\nu_{\alpha, \perp}$ (i.e. the e-folding rate of $\mathrm{v}_{\perp} 2$ ), which is (from the NRL Plasma Formulary):

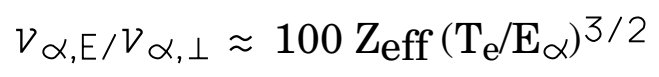

At the plasma axis, this ratio is $\approx 50$ for $3.5 \mathrm{MeV}$ alpha particles with $\mathrm{Te}(0)=5.5 \mathrm{keV}$ and $\mathrm{Z}_{\mathrm{eff}=3}$ (see Table 1). However, since in this code these collision times are assumed to be constant vs. radius, this ratio is taken to be characteristic of conditions near the SRD boundary $\left(\mathrm{n}_{\mathrm{e}}=2.7 \times 10^{13} \mathrm{~cm}^{-3}\right.$ and $\mathrm{T}_{\mathrm{e}} \approx 3.5 \mathrm{keV}$ ), i.e. $\tau_{\alpha, \mathrm{E}}=1 / \nu_{\alpha, \mathrm{E}}=0.1 \mathrm{sec}$ and $\tau_{\alpha, \perp}=1 / \nu_{\alpha, \perp}=10 \mathrm{sec}$ for all cases.

In Fig. 8 are maps of the alpha loss regions calculated by this code for the baseline case. At the top, the initial $3.5 \mathrm{MeV}$ alpha birth $\mathrm{R}$ and $X$ are varied for $\mathrm{z}=0$ (i.e. birth along the outer midplane). At the bottom, the initial alpha birth $R$ and $z$ are varied assuming $\cos X=0$ (i.e. birth at the banana tip). For both cases the orbits are followed for up to 75,000 toroidal transits using the collision rates noted above (i.e. for $\approx 0.1 \mathrm{sec}$ in real time), during which the alpha energy decreases by $1 / \mathrm{e}$ (i.e. from 3.5 to $1.3 \mathrm{MeV}$ ).

Three regions are highlighted in the maps of Fig. 8: the shaded prompt first-orbit loss region (alphas lost in fewer than 20 transits even without $\mathrm{TF}$ ripple present), the white confined region (alphas confined for at least 75,000 transits), and the " $\mathrm{X}$ " boundary region in which alphas are lost to the wall due to ripple loss occurring between 20 and 75,000 transits. In terms of banana tip locations, the ripple loss boundary is a circle of radius $\approx$ $20 \mathrm{~cm}$, i.e. smaller than expected from the collisionless GWB model due to the effect of collisional ripple loss. The corresponding ripple loss boundary radius for alphas lost within only 1000 transits is $\approx 30 \mathrm{~cm}$, similar to the result from the GWB modeling in Fig. 3. TF ripple trapping occurs only in a small region near the outer midplane, since most of the orbits with banana tips in the ripple wells are in the first-orbit region. 
The actual modeling of alpha ripple loss for this experiment was done using a Monte Carlo technique similar to that described in Ref. 15, in which $3.5 \mathrm{MeV}$ alphas are started at random pitch and toroidal angles with a radial distribution fit to the measured alpha source profiles. The alpha particle birth profiles in this experiment were very centrally peaked, with about $80 \%$ of the alpha banana tips born on initially confined orbits (i.e. within the white or X zones at the bottom of Fig. 8). For each type of plasma in Table 2, the ORBIT code was run using 1000 initial alphas for up to 75,000 toroidal transits each. The number of alphas in each run was limited by the available time on the VAX Alpha computers at PPPL (each run took about 2-3 weeks).

Results from the Monte Carlo modeling for the baseline I=1.4 MA case are shown in Fig. 9. Most of the ripple-lost alphas hit the first wall at poloidal angles between $0^{\circ}$ and $30^{\circ}$ below the outer limiter and at pitch angles near $60^{\circ}$, as expected from previous calculations using MAPLOS and ORBIT $[10,11,12,19]$. The alpha energy loss spectrum has a spike at the birth energy due to first-orbit loss (not shown), and a low energy tail due to collisional ripple loss. The total $\mathrm{TF}$ ripple loss fraction was $\approx 7 \%$ for this case, while the total first-orbit loss fraction was $\approx 15 \%$. Further results of the TF ripple modeling are discussed along with the experimental data in Sec. V.

\section{Experimental Results and Comparison with ORBIT Modeling}

This section contains a comparison between the midplane alpha loss measurements and results from ORBIT guiding center modeling of the alpha ripple loss for these experiments. All 34 DT discharges for the three plasma scans described in Sec. III are discussed, including the total alpha loss, pitch angle, and gyroradius distributions measured in the steady-state portion of these discharge (Secs. A, B, and C respectively). The time dependence and MHD effects on this data are described separately in Sec. D. Two separate data sets are presented for comparison purposes: one for 
plasmas with a smaller major radius $(\mathrm{R}=2.45 \mathrm{~m}$ instead of $\mathrm{R}=2.52 \mathrm{~m})$, and another for $\mathrm{R}=2.52 \mathrm{~m}$ plasmas taken near the end of the TFTR DT run (Secs. $\mathrm{E}$ and $\mathrm{F}$, respectively). Both of these additional data sets show the same qualitative but different quantitative features as the data of Secs. A-D, and so point out the sensitivity of the results to the limiter conditions. The uncertainties in the data and analysis are discussed separately (Sec. G).

The strategy of the analysis in this section is to attempt to compare the experimental results with the ORBIT code modeling, and to show where this modeling is successful and where it is deficient. The main deficiency is an inability of this model to explain the radial profile of the data, which leads to a discussion of the effect shadowing effect in Sec. VI.

\section{A. Neutron-Normalized Alpha Loss}

There were three plasma scans in this experiment: (a) the "q(r) scan" at $\mathrm{I}=1.4 \mathrm{MA}$ and $\mathrm{B}=4.7 \mathrm{~T}$, (b) the "q(a) scan" at $\mathrm{I}=1.4 \mathrm{MA}$ and $\mathrm{B}=4.7,3.9$ and $3.4 \mathrm{~T}$, and (c) the " $\rho$ scan" at constant $\mathrm{q}(\mathrm{a})$ at $\mathrm{B}=4.7,3.9$, and $3.4 \mathrm{~T}$ (see Sec. II). For each type of plasma the midplane alpha probe was moved to three different positions on three successive shots, namely, $\mathrm{r}_{\mathrm{ap}}=-2.0 \mathrm{~cm},-0.5 \mathrm{~cm}$, and $+1.0 \mathrm{~cm}$ with respect to the geometrical shadow of the outer limiter (see Table 3). One type of shot was common to each of these scans, namely the "baseline" case at $\mathrm{I}=1.4 \mathrm{MA}$ and $\mathrm{B}=4.7 \mathrm{~T}$.

Two examples of the raw scintillator image data from the midplane detector are shown in Fig. 10, one with $r_{a p}=-2.0 \mathrm{~cm}$ and the other with $r_{a p}=$ $+1.0 \mathrm{~cm}$, both for the baseline case. At the left are the scintillator light intensity patterns averaged over the steady-state period 4.5-4.8 sec during NBI, and at the right are the same data plotted in terms of the inferred pitch angle $X$ vs. gyroradius $\rho$. The alpha signals are clearly visible above the $(\mathrm{n}, \gamma)$-induced fiberoptic background [21], which can be seen within the square fiber bundle for the $\mathrm{r}_{\mathrm{ap}}=+1.0 \mathrm{~cm}$ case (the camera gain is $\approx 8$ times larger for the $r_{a p}=+1.0 \mathrm{~cm}$ case than the $r_{a p}=-2 \mathrm{~cm}$ case). The contours on the right represent a linear scale in alpha signal amplitude after background subtraction. The experimental pitch angles are measured with respect to the toroidal magnetic field at the detector, which is only $\approx 1^{\circ}$ less 
than the pitch angle with respect to the total magnetic field due to the large $q\left(a_{\text {lim }}\right)$ near the outer limiter (see Appendix 1).

The total alpha particle loss is defined as the total scintillator light emission integrated over the $X$ and $\rho$ region shown in Fig. 4 (nearly the same as the grid at the right of Fig. 5), minus the background flourescence of the fiberoptic bundle (see Sec. III), and averaged over the steady-state period 4.5 to 4.8 sec. The neutron-normalized alpha particle loss is this signal divided by the standard DT neutron rate monitor signal averaged over the same time period. From the raw data of Fig. 10, it can immediately be seen that the alpha loss is much larger per neutron for the $r_{a p}=-2.0 \mathrm{~cm}$ case, since the relative fiberoptic background level is much smaller. It can also be seen that the pitch distributions vary significantly with the aperture radial position.

The alpha collection fraction, i.e. the absolute value of the alpha loss into this detector divided by the global DT neutron rate, was estimated by comparing the midplane alpha loss signals with the $90^{\circ}$ detector signals for the baseline case. The alpha loss to the $90^{\circ}$ detector was assumed to be the first-orbit loss, as was found previously for similar DT discharges [13], and the alpha energy of the midplane probe signals was assumed to be $3.5 \mathrm{MeV}$ for this estimate. The resulting midplane alpha collection fraction vs. the detector aperture position for the baseline plasma case is shown in Fig. 11.

The inferred alpha collection fraction was in the range $\mathrm{F} \approx 10^{-6}$ for the $r_{a p}=-2.0 \mathrm{~cm}$ case to $F \approx 10^{-7}$ for the $r_{a p}=+1.0 \mathrm{~cm}$ case, i.e. the alpha flux into the detector was $\approx 10^{10}$ to $10^{11}$ alphas $/ \mathrm{sec}$ at a neutron rate of $\approx 10^{17} \mathrm{n} / \mathrm{sec}$. This alpha collection fraction is $\approx 10-100$ times larger than the expected firstorbit loss collection fraction for this detector, as calculated using the same Lorentz orbit code used for the $90^{\circ}$ alpha loss results [13]. Thus the firstorbit loss contribution to the midplane alpha loss signals was negligible. for the range of aperture positions used in this experiment. Note that the firstorbit loss of DD fusion products identified previously in the midplane probe signals was seen only for aperture positions $r_{a p}=+10 \mathrm{~cm}[10,11]$, where the $\mathrm{TF}$ ripple loss was much smaller (see Sec. VI). 
The relative variation of the neutron-normalized alpha loss for three plasma scans in this experiment is shown in Fig. 12, where the three plasma scans and three aperture positions are shown separately by normalizing the measured alpha loss for the baseline case at $r_{a p}=-0.5 \mathrm{~cm}$ to unity. These same variations in the data are shown in another way in Fig. 13, along with the total TF ripple loss calculated by the ORBIT code for each plasma type (also listed in Table 3). For Fig. 13, each aperture position the neutron-normalized alpha loss in the baseline case was normalized to the baseline global ripple loss calculated by ORBIT, so that the relative variations for the different types of $q(r)$ profiles can be seen. The shot-to-shot variability of the neutron-normalized alpha loss for a given plasma type and aperture position was only $\approx 5 \%$, and the estimated systematic uncertainty was about $\pm 10-20 \%$, mainly due to the background subtraction process (see Sec. V.F).

Looking first at the data and modeling results for the $r_{a p}=-0.5 \mathrm{c} \mathrm{m}$ cases, the following trends were observed with respect to the baseline:

a) $q(r)$ scan: the measured alpha loss increased by $\approx 50 \%$ for the current ramp-up case and decreased by $\approx 30 \%$ for the current ramp-down case, while the calculated global ORBIT ripple loss decreased by $\approx 6 \%$ and $17 \%$, respectively,

b) q(a) scan: the measured alpha loss decreased by $\approx 20 \%$ and $\approx 5 \%$ for the medium and low $\mathrm{q}(\mathrm{a})$ cases respectively, while the calculated ORBIT results decreased by $\approx 30 \%$ and $25 \%$, respectively,

c) $\rho$ scan: the measured alpha loss decreased by $\approx 40 \%$ and $\approx 60 \%$ respectively, while the calculated ORBIT results decreased by $\approx 20 \%$ and $\approx 10 \%$, respectively.

The qualitative trends at the other aperture positions were fairly similar, except for the low $q(a)$ case at $r_{a p}=+1.0$ which may have been affected by a stationary magnetic perturbation (see Sec. V.D). In general, the trends in the alpha loss data are not well explained by the ORBIT modeling. The variation in the measured alpha loss at a given aperture 
position was up to a factor of $\approx 4$ between different plasma types, but the range in the ORBIT-calculated global ripple loss was between $\approx 5-8 \%$, i.e. a variation of less than a factor of 2 . Also, the highest measured loss (for the current ramp-up cases) did not correspond to the highest calculated losses, and the lowest measured loss (for the low B constant q(a) case) did not correspond to the lowest calculated loss.

It is highly unlikely that these differences between experiment and modeling can be attributed to statistical uncertainty in the calculated alpha loss fractions, which was only about $\pm 0.5 \%$ for this 1000 particle sample (i.e. the results was thus $\approx 7.5 \pm 0.5 \%$ for the baseline case). There may be some additional systematic errors in the calculations, but these should be common to all cases. A study of the sensitivity of the global ripple loss to various modeling assumptions is given in Table 4 . The variation in the ORBIT code results for "collisionless" ripple loss (after only 5000 transits) was similar to that for the whole slowing-down calculation, as shown at the top of Fig. 13.

It is interesting that some of the trends in the global ORBIT code ripple loss results did not correspond well to initial expectations based on the SRD threshold, due to the limited physics contained in the SRD model (Sec. II). For example, in the $q(a)$ scan the ripple loss was expected to decrease with decreasing B (Fig. 3), but the ORBIT result increased slightly from medium $B$ to low $B$, while for the $\rho$ scan the ripple loss was expected to increase with lower B (higher $\rho$ ), but the ORBIT result decreased slightly at lower B. This is most likely due to the varying alpha source profiles, which tended to broaden at the lowest $\mathrm{B}$, and to the presence of a significant firstorbit loss, which depletes the alpha ripple loss region for the low-current cases.

The conclusion from this analysis is that the measured changes in the total alpha loss in these $q(r)$ and $\rho$ scans were not well explained by the ORBIT ripple loss calculations. Furthermore, the strong dependence of the measured alpha signals on the radial aperture position could not be predicted from the ORBIT code model, which assumed a smooth toroidal first wall. Although the absolute magnitude of the alpha loss to this 
midplane detector was larger than the first-orbit loss as expected from the $\mathrm{TF}$ ripple loss mechanism, the absolute level of alpha loss was not explained by the ORBIT modeling. Further analysis of this data requires a supplementary limiter shadowing model, as described in Sec. VI.

\section{B. Pitch Angle Variations}

The pitch angle of the alpha loss is important to help identify the loss mechanism, since the TF ripple loss is expected only in a relatively narrow range of pitch angles (see Fig. 3). Typical alpha particle pitch angle distributions measured by the midplane detector in this experiment are shown in Fig. 14. All these distributions are averaged over the gyroradius range $\rho=2-12 \mathrm{~cm}$ and over the time of $4.5-4.8 \mathrm{sec}$.

The top part of Fig. 14 shows the variation of the shape of these pitch angle distributions with probe position for the baseline case (I=1.4 MA, $\mathrm{B}=4.7 \mathrm{~T}$ ), in which the peak pitch angle moves from $X \approx 62^{\circ}$ at the aperture position $r_{a p}=+1.0 \mathrm{~cm}$ to $\chi \approx 54^{\circ}$ for $r_{a p}=-2.0 \mathrm{~cm}$. The bottom part of Fig. 14 shows that these pitch distributions vary considerably at the $r_{a p}=-2.0 \mathrm{~cm}$ position for different types of plasmas in this experiment. The variation is less strong at $\mathrm{r}_{\mathrm{ap}}=-0.5 \mathrm{~cm}$, where all of the distributions have a peak near $X \approx 60 \pm 2^{\circ}$, and all distributions have a very similar shape with a peak very near $\chi \approx 62^{\circ}$ at $\mathrm{r}_{\mathrm{ap}}=+1.0 \mathrm{~cm}$.

In general, these pitch distributions seem to consist of two distinct components: one near $X \approx 62-64^{\circ}$ (particularly dominant at the larger aperture radii), and another at $\chi \approx 52-54^{\circ}$ (particularly dominant at the smaller aperture radii). The instrumental resolution of $\approx 6^{\circ} \mathrm{FWHM}$ limits the ability to distinguish between these two components, and there is a potential systematic alignment uncertainty of $\pm 3^{\circ}$. These pitch angles are measured with respect to the toroidal field, as before [10-12].

The pitch angle distributions calculated for global TF ripple loss by the ORBIT code are shown at the top of Fig. 15 for the two of the cases from the bottom of Fig. 14. The calculated pitch angle range for TF ripple loss is 
roughly the same for all the plasmas in this experiment (to within the statistical uncertainties), namely within $x \approx 54^{\circ}-68^{\circ}$. These distributions include all the TF ripple lost alphas at various energies and poloidal angles near the outer midplane (Fig. 3), and are measured with respect to the total magnetic field at the wall, which makes them $\approx 1^{\circ}$ larger than when measured with respect to the toroidal field.

The trajectories of $\mathrm{E}=2.5 \mathrm{MeV}$ alpha orbits entering the detector with pitch angles near the ends of this range are shown at the bottom of Fig. 15. The orbit with $\chi \approx 54^{\circ}$ has a banana tip in the low $\mathrm{TF}$ ripple region close to the magnetic axis, while the orbit with $\chi \approx 68^{\circ}$ has its banana tip in the high $\mathrm{TF}$ ripple region (the ripple is turned off for these orbits). The corresponding orbits for alpha of lower (higher) energies are at a higher (lower) vertical position, but have nearly the same major radius, except for the orbits $x \leq 54^{\circ}$ with $\mathrm{E} \approx 3.5 \mathrm{MeV}$ which are near the passing-trapped boundary. Therefore all alpha particles lost due to TF ripple in the ORBIT modeling are on trapped orbits on their last bounce before hitting the wall.

The variation of the peak pitch angles vs. aperture radius is shown in Fig. 16, along with the range of pitch angles predicted for TF ripple loss by the ORBIT code. All the data falls within the calculated range of $\chi \approx 54^{\circ}-68^{\circ}$ for TF ripple loss. However, the code does not explain the systematic variation of the data with the aperture radius. Furthermore, the statistical uncertainties in the code results are too large to explain the differences among the pitch angle distributions for the various examples in Fig. 14.

The conclusion from this comparison is that the measured pitch angle distributions are roughly consistent with $\mathrm{TF}$ ripple loss, but their variations with $q(r)$ can not be explained by the ORBIT code. Further analysis of this data using a supplementary limiter shadowing model is described in Sec. VI.

C. Gyroradius (Energy) Variations 
The alpha gyroradius distributions can in principle be used to determine the energy spectrum of the alpha loss, which can be compared with the predictions from the ORBIT code (Fig. 9). However, the design of the detectors was optimized for pitch angle and not for gyroradius resolution, so in practice only the average energy of the alpha loss can be determined and not its energy distribution function.

Examples of the measured lost alpha gyroradius distributions for this experiment are shown in Fig. 17, where in each case the data was averaged over the pitch angle range $X=45-75^{\circ}$ between $4.5-4.8$ sec (see Sec. III). At the top of this figure are the distributions for the three shots in the $q(r)$ scan, all for the same aperture position of $r_{a p}=-2.0 \mathrm{~cm}$ and for $B=4.7 \mathrm{~T}$. At the bottom of are the measured distributions for the three shots in the $q(a)$ scan, again for $r_{a p}=-2.0 \mathrm{~cm}$. There is a relatively large instrumental spread of the data in the gyroradius centroid coordinate direction due to the finite size of the detector apertures; thus the average alpha gyroradius can only be estimated by comparing the data with modeled distributions based on the detector aperture and scintillator geometry, three of which are also shown at the top of Fig. 17.

The three shots of the $q(r)$ scan at the top of Fig. 17 have the same gyroradius distribution shapes, implying that the energy spectrum of the alpha loss was not sensitive to this $\mathrm{q}(\mathrm{r})$ variation. The best fit is for $\mathrm{E}=2.5$ $\mathrm{MeV}$ alphas, but the systematic uncertainty in the gyroradius centroid coordinate is about $\pm 1 \mathrm{~cm}$ (see Sec. V.F), so the results at full field are consistent with the loss of $E=2.5 \pm 1 \mathrm{MeV}$ alphas. The three shots of the $q(a)$ scan at the bottom of Fig. 17 show an increase in the gyroradii with decreasing $\mathrm{B}$, but this is mainly due to the change in $\mathrm{B}$ itself.

The peak gyroradii during the steady-state period 4.5-4.8 sec for all plasmas in this experiment are shown in Fig. 18 as a function of B, along with model calculations for various energies. The inferred average alpha loss energy appears to be $\mathrm{E} \approx 2.5 \pm 1.0 \mathrm{MeV}$ for the $\mathrm{B}=4.7 \mathrm{~T}$ cases, and $\mathrm{E} \approx 2 \pm 1$ $\mathrm{MeV}$ for the $3.9 \mathrm{~T}$ and $3.4 \mathrm{~T}$ cases. However, the instrumental uncertainties at lower B and larger gyroradii are higher (see Sec. V.F). 
The strongest conclusion from this analysis is that the energy of the alpha loss is insensitive to both the type of plasma and the aperture position in this experiment. However, the relatively poor energy resolution and systematic uncertainties in this measurement make a quantitative comparison with the ORBIT results difficult. The best estimate is that the average energy of alpha loss is similar to the mean energy of the TF ripple loss of $\mathrm{E} \approx 2.5-3.0 \mathrm{MeV}$ (Fig. 9), but with uncertainty which brackets the whole range of expected alpha energy spectrum, i.e. $\mathrm{E}=2.5 \pm 1 \mathrm{MeV}$.

\section{Time Dependence and the Effects of MHD Activity}

A typical time dependence of the midplane alpha loss signal with respect to the neutron signal (i.e. alpha source rate) was shown in Fig. 1. For almost all plasmas and aperture positions, the relative alpha loss per neutron is constant (to within about $\pm 20 \%$ ) during the time interval between $\approx 50$ msec after the start of NBI and 200 msec after the end of NBI, similar to the behavior seen previously for a I=2.0 MA case [12]. There is no clear correlation between the relative alpha loss per neutron after NBI and the magnitude of the total neutron-normalized alpha loss during NBI.

However, there are small changes in the pitch angle and gyroradius distributions between the early-NBI and the post-NBI phases, as illustrated at the top of Fig. 19 for the baseline plasma case with $r_{a p}=-0.5 \mathrm{~cm}$. The most interesting of these is a shift in the gyroradius distribution toward lower energies after the end of NBI, such as might be expected for a delayed alpha loss component due to collisional TF ripple diffusion. The location of the peak of the gyroradius distribution vs. time is shown for some of the baseline case at the bottom of Fig. 19. The decrease in gyroradius $\approx 200 \mathrm{msec}$ after the end of NBI for the $r_{a p}=-0.5 \mathrm{~cm}$ case corresponds to an energy decrease of $\approx 20 \%$ with respect to the steady-state loss energy.

The expected time dependence for collisional TF ripple loss can be estimated from the energy spectrum of ripple loss calculated by the ORBIT code (Fig. 9). For all cases, $\approx 2 / 3$ of the collisional TF ripple loss occurred within $\approx 30$ msec of the alpha birth at $3.5 \mathrm{MeV}$, i.e. within $\approx 1 / 3$ of an energy 
slowing-down time. This is very fast with respect to the $\approx 250$ msec rise time of the alpha source rate during NBI (Fig. 1), so is consistent with the constant alpha loss per neutron observed during NBI (e.g. Fig. 1). After NBI ends the global alpha source rate e-folds in only $\approx 75 \mathrm{msec}$, so an increase in the alpha loss per neutron of $\approx 60 \%$ is expected $\geq 0.1 \mathrm{sec}$ after the end of NBI (from a numerical estimate). This increase is larger than the $\leq 20 \%$ increase observed in the data. However, interpretation of this difference requires a time dependent analysis of the ripple loss, including evolution of the equilibrium, source profile, and collisionality after the end of NBI, which is not possible with the present ORBIT code.

Some of the discharges in this experiment had relatively large sawteeth during NBI, which are common in TFTR at low NBI power and low $q(a)$. However, in all these sawtoothing cases the alpha loss per neutron was constant except for a very brief $(\approx 100 \mu \mathrm{sec})$ increase by a factor $\leq 3$ of alpha loss just at the sawtooth crash, similar to that seen previously for DD fusion products [22]. This burst had a negligible effect of the alpha loss averaged over 4.5-4.8 sec, so does not affect the interpretation of the signals of Sec. V.A-C.

The only other visible MHD effect was a surprisingly strong influence of stationary magnetic perturbations (SMPs) on the alpha loss signals. SMPs (sometimes called "locked modes") are measured on TFTR by a pair of radial field coils [23], and are large-scale non-rotating edge magnetic perturbations which occurred for most of the current ramp-down discharges in this experiment. The discharges with an SMP had a significantly lower ion and electron temperature and a lower DT neutron rate that the ones without an SMP. The main effect of the SMP was to drastically change the pitch angle distribution of the alpha loss, as shown in Fig. 20, where for an aperture position of $r_{a p}=-0.5 \mathrm{~cm}$ the peak pitch location shifted from $\approx 62^{\circ}$ without the SMP to $\approx 51^{\circ}$ with the SMP. The total alpha loss decreased by up to $\approx 20 \%$ with SMPs, leading to the scatter in the ramp-down data of Figs. 12 and 13. Subsequent discharges made with slower current ramp-down rates also had SMPs, but no SMPs were seen in the 1.8 MA->1.4 MA ramp-down cases, or in other discharges in this experiment. 
These MHD effects on the alpha loss can not be understood without a set of ORBIT calculations which include these low-n magnetic perturbations. Such a study is planned, but is beyond the scope of this paper.

\section{E. Small Major Radius Cases}

For comparison purposes, a radial probe scan was made in $\mathrm{R}=2.45 \mathrm{~m}$ plasmas with the same plasma current and toroidal field as the $R=2.52 \mathrm{~m}$ plasmas discussed above (i.e. $\mathrm{I}=1.4 \mathrm{MA}$ and $\mathrm{B}=4.7 \mathrm{~T}$ ). These smaller major radius plasma allowed a larger inward scan of the probe aperture to $r_{a p}=-$ $6.5 \mathrm{~cm}$, as shown in Fig. 21, since the plasma edge was farther from the limiter shadow at the probe ( $34 \mathrm{~cm}$ vs. $19 \mathrm{~cm}$ for $\mathrm{R}=2.52 \mathrm{~m}$ plasmas).

The alpha loss measured for the $\mathrm{R}=2.45 \mathrm{~m}$. plasmas increased as the probe was moved inward until $r_{a p}=-5 \mathrm{~cm}$, i.e. $3 \mathrm{~cm}$ farther inward than any point in the probe scan used for the $R=2.52 \mathrm{~m}$ plasmas. The level of the normalized alpha loss per neutron for the $r_{\mathrm{ap}}=-0.5 \mathrm{~cm}$ position was within $5 \%$ of that for the baseline $R=2.52 \mathrm{~m}$ plasmas, but the signal at $r_{a p}=-2.0 \mathrm{~cm}$ for the $R=2.45 \mathrm{~m}$ plasma was a factor of $\approx 2$ lower than for the $\mathrm{R}=2.52 \mathrm{~m}$ plasma (the signal at $\mathrm{r}_{\mathrm{ap}}=+1 \mathrm{~cm}$ was too small to measure above the fiberoptic background level for $\mathrm{R}=2.45 \mathrm{~m}$ ). As the detector was moved further inward for the $R=2.45 \mathrm{~m}$ case, the loss saturated at about $r_{a p}=-5 \mathrm{c} \mathrm{m}$ at a factor of 4 above the level at $r_{a p}=-2.0 \mathrm{~cm}$.

The pitch angle distributions for the $\mathrm{R}=2.45 \mathrm{~m}$ plasmas are shown at the bottom of Fig. 21, all normalized to the same height to show the changes in shape vs $r_{a p}$. The case at $r_{a p}=-0.5 \mathrm{~cm}$ had a pitch angle distribution peaked at $54^{\circ}$, i.e. significantly lower than the corresponding $62^{\circ}$ peak in the $\mathrm{R}=2.52 \mathrm{~m}$ case (Fig. 14). The component of the pitch distribution at $62^{\circ}$ in the $\mathrm{R}=2.45 \mathrm{~m}$ cases was largest for the farthest outward position, and decreased as the probe moved inward, similarly to the $R=2.52 \mathrm{~m}$ cases. 
The gyroradius distributions were analyzed as in Sec. V.C, and the result was a peak gyroradius at $6.2 \pm 0.1 \mathrm{~cm}$, i.e. nearly identical to the corresponding $R=2.52 \mathrm{~m}$ cases at the same toroidal field. There was little or no time dependences of the gyroradius peak in the $R=2.45 \mathrm{~m}$ cases.

In summary, the alpha loss for the smaller major radius plasmas showed some features similar to the $R=2.52 \mathrm{~m}$ plasmas described in Secs. V.A-C; namely, the pitch angle and gyroradius distributions were similar, and the alpha signal levels increased strongly as the probe was moved inward. Although it is expected that the global TF ripple loss is smaller for the $\mathrm{R}=2.45 \mathrm{~m}$ plasmas due to the lower average ripple level, the measured ratio of the alpha loss per neutron between the two cases depended strongly on the probe aperture position. This makes it clear that before any quantitative connection can be made between these midplane measurements and $\mathrm{TF}$ ripple loss modeling, the origin of the radial dependence of the alpha loss signals needs to be better understood. This is discussed in Sec. VI.

\section{F. Sensitivity to Limiter Geometry}

Near the end of the TFTR DT run an extended midplane probe scan at $\mathrm{R}=2.52 \mathrm{~m}$ was made to obtain a more complete radial profile (these data was taken about 1.5 years after those in Sec. A-E). In this extended scan the aperture was moved inward by an additional $4.5 \mathrm{~cm}$ between $r_{a p}=-6.5 \mathrm{~cm}$ and $+1 \mathrm{~cm}$ in plasmas which had external parameters essentially the same as for the baseline case.

Although the results described in Secs. IV.A-C were highly reproducible during the period in which they were taken, the results of this extended probe scan were considerably different, as shown in Fig. 22. At $\mathrm{r}_{\mathrm{ap}}=-2 \mathrm{~cm}$ the total alpha flux (per neutron) was $\approx 4$ times smaller and had a different pitch angle distribution for the later scan, and the radial efolding length within the region of overlap was longer by a factor of $\approx 3$. The qualitative behavior of the new extended scan was similar to that measured previously for $\mathrm{R}=2.45 \mathrm{~m}$ (Fig. 21); namely, the alpha loss increased as the aperture was moved in to at least $r_{a p}=-5 \mathrm{~cm}$, and the pitch 
angle distribution shifted to lower angles as the aperture was moved inward.

This unexpected difference was most likely due to repositioning of the $\mathrm{RF}$ antennas which occurred just before the extended scan. These RF antennas were located between the RF limiters at the outboard midplane, and part of their structure may have been at a smaller major radius than the RF limiters for the extended scan (their exact position uncertain by about $\pm 1 \mathrm{~cm}$ ). Even without understanding the specific mechanism of this difference, its general implication is that the midplane alpha loss signals are very sensitive to the limiter position, as discussed further in Sec. VI.

\section{G. Uncertainties}

The main uncertainties in the alpha loss measurements are related to the fiberoptic background subtraction and to the detector geometry. The main experimental uncertainties in the TF ripple loss calculations relate to the profiles of $q(r)$ and the alpha source $S_{\alpha}(r)$.

The uncertainty due to the fiberoptic background subtraction depends on the probe position, as illustrated in Fig. 10 for two of the standard cases. The net signal level divided by the fiberoptic background level are $\mathrm{s} / \mathrm{b} \approx 2$ for $\mathrm{r}_{\mathrm{ap}}=-0.5 \mathrm{~cm}$ (\#87473), $\mathrm{s} / \mathrm{b} \approx 8$ for $\mathrm{r}_{\mathrm{ap}}=-2.0 \mathrm{~cm}(\# 87474)$, and $\mathrm{s} / \mathrm{b} \approx+1.0$ for $\mathrm{r}_{\mathrm{ap}}=$ $+1.0 \mathrm{~cm}$ (\#87475). The uncertainty in this background level is estimated to be $\pm 10 \%$ from the uniformity of background-only images taken when the probe is retracted. Thus the uncertainty in the total alpha loss due to the background subtraction process is about $\pm 5 \%$ for $r_{a p}=-0.5 \mathrm{~cm}, \pm 10 \%$ for $r_{a p}=$ $-2.0 \mathrm{~cm}$, and $\pm 20 \%$ for $r_{\mathrm{ap}}=+1.0 \mathrm{~cm}$. There is an additional systematic error in the total alpha loss measurement due to the detector cut-off at $\rho \approx 12 \mathrm{~cm}$ (see Fig. 4), which causes an underestimate of the loss for the lower-B cases. This was estimated from the measured gyroradius distributions to be a relative error of $\approx 5 \%$ between the lowest and highest $B$. The shot-to-shot reproducibility of the total alpha loss was within $\pm 5 \%$ for shots with the same plasma conditions. Combining these three sources of error, the relative uncertainty among these alpha loss measurement is about $\pm 10 \%$ 
for the cases with low background levels $\left(r_{a p}=-0.5 \mathrm{~cm}\right.$ and $\left.\mathrm{rap}=-2.0 \mathrm{~cm}\right)$, and $\pm 25 \%$ for the high background cases at $r_{a p}=+1.0 \mathrm{~cm}$.

The results of the above analysis are indicated in Fig. 13 by the $\pm 10 \%$ error bar on the relative alpha loss at $r_{a p}=-0.5 \mathrm{~cm}$. The total alpha loss was also cross-checked with the light signal from the photomultiplier tube which integrated over most of the fiberoptic bundle. The relative alpha signals evaluated by the PM tube for the seven cases at $r_{a p}=-2.0 \mathrm{~cm}$ were all within about $\pm 5 \%$ of that evaluated by the camera (Sec. V.A).

The absolute flux estimated for Fig. 11 has additional uncertainties. The normalization of the midplane signals to the $90^{\circ}$ detector signals is relatively accurate $( \pm 20 \%)$, but the absolute calibration of the $90^{\circ}$ signals, on which the absolute midplane flux was based, was uncertain by a factor of $\approx 2$ [13]. In addition, the light output of the $\mathrm{P} 46$ scintillator is proportional to the energy of the alpha particle, so the inferred alpha flux depends on the assumed alpha energy spectrum. The energy used for Fig. 11 was $3.5 \mathrm{MeV}$, but this is known only to be $2.5 \pm 1 \mathrm{MeV}$. Therefore the uncertainty in the absolute flux estimate is about a factor of 3 .

The pitch angle and gyroradius distributions discussed in Secs. V.B and V.C are subject to potential systematic errors in the knowledge of the detector geometry and alignment. An estimated uncertainty of \pm 1 pixel $(\approx \pm 0.1 \mathrm{~cm})$ in the compressed camera images of the $2.5 \mathrm{~cm} \times 2.5 \mathrm{~cm}$ scintillator translates into a absolute uncertainty of $\pm 3^{\circ}$ in pitch angle and $\pm 1 \mathrm{~cm}$ in gyroradius. The latter translates into the $\pm 1 \mathrm{MeV}$ uncertainty in the inferred alpha energy shown in Fig. 18. The relative uncertainties are roughly one-third of this (the size of the uncompressed pixels). The effect of optical vignetting on the location of the peak in the gyroradius distributions was taken into account in the analysis, based on a white-plate calibration of the system during construction [17].

The position of the detector probe was controlled by a stepper motor to an accuracy of $\pm 0.02 \mathrm{~cm}$ with respect to the vessel. The location of the edge of the limiters with respect to the vessel was known to an accuracy of \pm 0.3 $\mathrm{cm}$. 
The calculations of $\mathrm{TF}$ ripple loss are sensitive to uncertainties in the $q(r)$ and neutron source profiles $S_{\alpha}(r)$, as discussed in Sec. IV. The estimated uncertainty in the MSE $q(\mathrm{r})$ profile measurements are $\pm 5 \%$ at $\mathrm{q}(0)$ and $q(a)$, and $\pm 10 \%$ near the middle of the plasma at $r / a \approx 0.5$ [14]. The alpha source profiles measured by the multichannel neutron collimator were parameterized by $\mathrm{S}_{\alpha}(\mathrm{r}) \propto\left[1-(\mathrm{r} / \mathrm{a})^{2}\right]^{\mathrm{Sexp}}$. The estimated uncertainty in the evaluation of $S_{\exp }$ was \pm 1 for typical values of $S_{\exp }=6$ (see Table 2). The sensitivity of the ORBIT calculations of TF ripple loss in TFTR has been discussed elsewhere [15,19], and some examples of these sensitivities for the baseline case are shown in Table 4 . The result is that the results of these ORBIT calculations of TF ripple loss are uncertain to about $\pm 1 \%$, e.g. for the baseline case, the global TF ripple loss is $\approx 7.5 \pm 1 \%$.

\section{Limiter Shadowing Effects}

The attempts made in Sec. V to explain the midplane alpha loss measurements based on the global ORBIT code ripple loss modeling were not very satisfactory. In addition to the experimental and modeling uncertainties discussed in Sec. V.E, there were two generic problems:

1) the statistics of the ORBIT code results were too sparse to calculate the expected variations in TF ripple loss at the local detector position,

2) the ORBIT code assumed a smooth toroidal first wall, and so could not be used to interpret the strong radial variations of the detected signal.

The purpose of this section is to clarify how the actual highly nonsmooth limiter geometry can affect the interpretation of the local detector signals. This occurs when to the limiter at the outer wall of TFTR have a "shadowing" effect, i.e. when the escaping alpha orbits hit the outer limiters before the can reach the detector aperture. In this section the results of the ORBIT code are supplemented by some preliminary modeling of this limiter shadowing effect, but these modifications are not yet accurate or complete enough to quantitatively explain the experimental data. 
Possible methods of further improving the modeling are discussed in Sec. VII.

\section{A. Orbit Gaps and Jumps Near the Outer Midplane}

The basic geometry of the limiter shadowing effect in TFTR is shown in Fig. 23. Without any TF ripple in the guiding center ORBIT calculation, a $3.5 \mathrm{MeV}$ alpha orbit which is launched backward in time from the detector aperture at $r_{a p}=-2.0 \mathrm{~cm}$ crosses the geometrical shadow of the outer limiter in the upper half of the vessel before completing one poloidal transit. Since there are several outer midplane limiters in TFTR (Fig. 5), and since $q(r) \approx 20-30$ near the outer limiter (see Appendix 1), almost all alpha orbits which cross the outer midplane limiter shadow will hit the limiter before entering the detector aperture (in the absence of $\mathrm{TF}$ ripple diffusion).

Thus there is a minimum radial "gap" over which a previously confined alpha particle orbit must "jump" before it can reach the detector aperture, even when the aperture is radially inside of the geometrical shadow of the limiter at $r_{a p}=-2.0 \mathrm{~cm}$. This gap $\Delta_{r}$ is determined by the aperture location and the shape of the marginally confined alpha orbit at a given energy and pitch angle, as shown in Fig. 23. If the radial jumps $\delta_{\mathrm{r}}$ are diffusive, and if the aperture is inside the gap region, i.e. $\Delta_{r}>0$, the detected ripple loss signal should decrease with increasing $r_{a p}$. The expected profile for first-orbit loss should be nearly flat in this region since the radial jumps are not diffusive, but occur on a single large step.

Fig. 24 shows ORBIT calculations of typical radial gaps between the detector aperture at $\mathrm{r}_{\mathrm{ap}}=0 \mathrm{~cm}$ and marginally confined alpha orbits of the same pitch angle and energy. These marginally confined orbits all intersect the limiter at the outer midplane over this range of energy and pitch angle. The gaps were calculated numerically for the baseline case using approximate values of the $q$ and Shafranov shift at the outer limiter, based on an analysis of the vacuum fields (see Appendix 1). For example, for alphas of $\mathrm{E}=2.5 \mathrm{MeV}$ and $X \approx 60^{\circ}$ the gap is $\triangle_{\mathrm{r}} \approx 5 \mathrm{~cm}$, i.e. for all detector locations with $r_{a p} \geq-5 \mathrm{~cm}$ alpha orbits entering the aperture at this energy 
and pitch angle will intersect the limiter in the absence of radial diffusion. For lower energy and lower pitch angle alphas the gaps tend to be smaller since the shifts of their banana orbits from the flux surfaces are smaller.

Also shown in Fig. 24 are more accurate calculations of the gaps for $\mathrm{E}=1.5 \mathrm{MeV}$ alphas made using a Lorentz orbit code which includes a full 2$D$ model of the magnetic flux surfaces. The gaps are $\approx 1 \mathrm{~cm}$ smaller than for the approximate ORBIT calculations, and become less than $\triangle \mathrm{r} \approx 2 \mathrm{~cm}$ at pitch angles below $X \approx 60^{\circ}$. The effective gaps are further reduced by the $\approx 0.6$ $\mathrm{cm}$ radial wobble of the total magnetic field due to the TF ripple near the outer midplane, which was not present in either calculation. Therefore alphas with low enough energy $(\mathrm{E}<2 \mathrm{MeV})$ and pitch angle $\left(X<60^{\circ}\right)$ are likely to be unshadowed by the limiter at $r_{a p}=-2 \mathrm{~cm}$, depending on the exact vacuum fields (see Appendix 1).

The extent to which alphas can cross this gap on their last poloidal transit depends on their radial "jumps" due to the ripple. These jumps occur at the banana tips with a vertical displacement of approximately [1]:

$$
\delta_{\mathrm{V}} \approx(\mathrm{N} \pi / \sin \theta)^{1 / 2}(\mathrm{q} / \varepsilon)^{3 / 2} \rho \delta \sin (\mathrm{N} \phi)
$$

where $\theta$ and $\phi$ are the poloidal and toroidal angles of the banana tip (see under Eq. 1 for other definitions). The physical cause of these jumps is the change in the alpha drift motion caused mainly by the ripple in the magnitude of $B$ near the banana tips. These jumps are oscillatory for small ripple, but become random when the particle "islands" in banana tip phase space (e.g. $r, \theta)$ begin to overlap [1,2]. For almost all alphas entering the detector the SRD threshold criterion (Eq. 1) is satisfied and these jumps are effectively random on successive banana tips.

Typical magnitudes of these SRD-induced jumps for $3.5 \mathrm{MeV}$ alphas are also shown in Fig. 24, both from a numerical calculation using the ORBIT code and from the analytic formula of Eq. 3. These jumps are typically $\delta \mathrm{r} \approx 3 \mathrm{~cm}$ for $X \approx 60^{\circ}$, i.e. comparable to the radial gaps (the radial jumps as measured at the outer midplane are 2-3 times larger than the corresponding vertical jumps at the banana tip due to the shape of the 
banana orbit). For a given alpha energy the jumps increase rapidly with pitch angle as the banana tip location falls in a higher ripple region. There are two such jumps between the marginally confined orbit and the detector aperture. Thus there is a significant probability that alphas undergoing SRD can jump the gaps and enter the detector aperture.

\section{B. Probability of Missing the Limiter}

The ORBIT code with the TF ripple present was used to estimate the probability that SRD allowed the alphas to jump these gaps. Alphas were launched at the detector aperture and allowed to move "backwards in time" into the plasma, and the fraction of alphas which missed the limiter after their first poloidal transit was taken as the probability of alphas reaching the detector from the plasma. An example of such an alpha orbit was shown in Fig. 7. For alphas of a given energy and aperture location, 1000 alpha orbits were launched at each pitch angle with varying toroidal angles to simulate the alphas which are actually diffusing outward due to TF ripple diffusion.

Results of this simulation for the baseline case are shown in Fig. 25. For the aperture position $\mathrm{r}_{\mathrm{ap}}=0 \mathrm{~cm}$ (at the top), the probability of missing the limiter on the first poloidal transit is found to increase with increasing pitch angle, as expected from the larger jumps at higher pitch shown in Fig. 24. At pitch angles between $X=55-65^{\circ}$ the probability of missing the limiter also increases with decreasing energy at a given pitch angle, since the gaps are smaller (the jumps decrease linearly with gyroradius according to Eq. 3, but the banana tip locations move to larger ripple as the energy is reduced for a given pitch angle).

For a typical energy of $\mathrm{E}=2.5 \mathrm{MeV}$ the probability of missing the limiter varies with the assumed aperture position as shown at the bottom of Fig. 25. At $\chi=50^{\circ}$ the probability increases sharply from nearly 0 to 1 at the edge of the gap, since the jumps are very small or zero for these orbits near the SRD boundary. At $\chi=60^{\circ}$ the probability starts to increase only for $\mathrm{r}_{\mathrm{ap}}<-$ $2 \mathrm{~cm}$, and for $X=70^{\circ}$ the probability is approximately independent of the $r_{a p}$ 
since the jumps are so large. Thus the limiter shadowing probability calculated in this way is a sensitive function of aperture position, energy, and pitch angle.

These results are qualitatively similar to previous calculations of the limiter shadowing effect done to simulate the midplane probe signals in $\mathrm{I}=2.0 \mathrm{MA}$ discharges [11, 18]. However, in Ref. [11] the magnetic geometry was highly simplified (no Shafranov shift), so the gaps were not as well calculated as here, and in [18] the limiter shadowing probability per poloidal transit was approximated by a single probability independent of energy and pitch angle. The present probability model is also incomplete and quantitatively inaccurate since it evaluates the limiter intersection only on the first poloidal transit, and does not attempt to calculate the history of the alpha orbit backward all the way back to its birth (see Sec. VIID).

\section{Comparisons with Pitch Angle and Radial Profile Data}

The probabilities of jumping the gaps as calculated in Sec. B can be used to estimate the effect of limiter shadowing on the baseline case. Some of the data can be understood qualitatively through this model, but the uncertainties are too large to allow a quantitative comparison.

The change in the expected ripple loss pitch angle distributions due to the limiter shadowing effect is shown in Fig. 26. The model curves are the baseline ORBIT code pitch angle distributions (Fig. 15) multiplied by the probability of missing the limiter as a function of pitch angle, assuming $\mathrm{E}=2.5 \mathrm{MeV}$. The main effect of the shadowing is to shift the expected distribution to a higher pitch angle, since the probability of missing the limiter increases rapidly with pitch angle. For the aperture position $r_{a p}=-$ $0.5 \mathrm{~cm}$ the resulting "ORBIT+shadowing" model distribution peaks close to the measured peak of the distribution shown at $\chi \approx 64^{\circ}$. However, the data for $\mathrm{r}_{\mathrm{ap}}=-2.0 \mathrm{~cm}$ peaks at $\chi \approx 54^{\circ}$, while the calculated pitch distribution even for $r_{\mathrm{ap}}=-3.5 \mathrm{~cm}$ still peaked near $X \approx 64^{\circ}$. Therefore another mechanism besides SRD must cause the observed low pitch angle peak at $\chi \approx 54^{\circ}$ in the distribution for $\mathrm{r}_{\mathrm{ap}}=-2.0 \mathrm{~cm}$. 
To explore this comparison further, it is useful to decompose the measured pitch angle distribution into two components, as suggested by the results of Fig. 14; namely, one component centered at low pitch angles of $X \approx$ $54^{\circ}$ and the other at high pitch angles of $\chi \approx 64^{\circ}$, both with a width of $\pm 6^{\circ}$ set by the instrumental resolution. The resulting radial profiles of the data for the baseline case are compared with the radially resolved probability of missing the limiter in Fig. 27. For high pitch angles, the probability increases as the aperture moves toward the plasma because the gap becomes smaller and so a radial jump across the gap becomes more likely. The data for $X \approx 64^{\circ}$ can be fairly well fit by the SRD jump model for an alpha energy of $\mathrm{E}=1.5-2.5 \mathrm{MeV}$. For the lower pitch angles, the increase in probability occurs only when the gap becomes zero, i.e. when orbits traced backward from the aperture are unshadowed by the limiter. At $X=54^{\circ}$ this seem to require an energy of $\mathrm{E}<1.5 \mathrm{MeV}$ to fit the data, but the location of this boundary is uncertain due to its dependence on the vacuum fields (see Appendix 1). Thus it is likely that the dominant low pitch angle feature at $r_{a p}=-2 \mathrm{~cm}$ is due to the collection of "confined" alphas, which only occurs when the aperture is far enough in so that there is no limiter shadowing The expected flux for this process is estimated in Sec. VI.D.

The relatively good fits between the pitch angle distributions and radial profiles for the outward aperture positions and higher pitch angles suggests that this model might explain the differences between the data and the ORBIT modeling among the various $r_{a p}=+1.0 \mathrm{~cm}$ cases (Fig. $13 \mathrm{~b}$ ), all of which had a high pitch angle (Fig. 16). Qualitatively, there are two limiter shadowing effects which vary with plasma type: the size of the SRD jumps increases linearly with $q^{3 / 2} \rho$ (see Eq. 3), and the size of the gaps varies with the outer limiter $q$ and Shafranov shift (see Appendix 1). The first effect causes the jumps to decrease by $\approx 15 \%$ as $B$ is lowered in the $q(a)$ scan and to increase by $\approx 40 \%$ as $B$ is lowered in the $\rho$ scan. Neither trend is in the direction of explaining the deviations of the data at $r_{a p}=+1.0 \mathrm{~cm}$ from the scaled ORBIT model results of Fig. 13. And although the vacuum fields did vary with the type of plasma, the calculated limiter shadowing probabilities for the $\mathrm{r}_{\mathrm{ap}}=+1.0 \mathrm{~cm}$ cases did not tend to explain the trends in the data. The uncertainties in the vacuum fields at the limiter and in the 
energy spectrum of the alpha loss are too large to allow quantitative explanation of the present data.

\section{Finite Gyroradius Effects}

One factor not taken into account in the limiter shadowing model above was the finite gyroradius of the alphas, which for this experiment was in the range $\rho \approx 5-11 \mathrm{~cm}$. This is comparable to the size of the jumps and gaps in the guiding center modeling, so could be important in determining the alpha flux to the detector near the shadow of the limiter.

Two examples of finite-gyroradius alpha orbits launched from the detector backward in time toward the limiters are shown in Fig. 28. For these calculations a version of the Lorentz finite gyroradius code [25] was used in which the magnetic fields were taken from the full 2-D equilibrium for the baseline case (not including the TF ripple). Both orbits started at the aperture location $r_{a p}=-2 \mathrm{~cm}$ with a gyrophase angle defined by the detector aperture pair (see Fig. 4). The orientation of the small $\left( \pm 2^{\circ}\right)$ gyrophase acceptance range of the detector was designed to accept alpha orbits only near the outermost major-radial excursion.

As shown in Fig. 28, the alpha orbit starting out with $\mathrm{E}=2.5 \mathrm{MeV}$ and $\chi=60^{\circ}$ intersected the $\mathrm{RF}$ limiter near the outer midplane (see Fig. 5), confirming that this orbit is within the effective limiter shadow even when the aperture was $2 \mathrm{~cm}$ inside of the geometrical shadow of the limiters. The limiter was accurately modeled in this code as a full poloidal ring with a toroidal extent of $54 \mathrm{~cm}$ and a toroidal radius of curvature of $155 \mathrm{~cm}$. Since the alpha gyro orbit length is typically $20 \mathrm{~cm}$ in the toroidal direction, this limiter blocks all alpha orbits which have their outermost radial excursions beyond alim=99 $\mathrm{cm}$. Thus the finite gyroradius effect itself does not significantly change the calculation of the gaps in Sec. V.A, since the alphas enter the detector only at outermost point of their gyrophase.

The gap sizes as calculated by this Lorentz code decreased as the alpha energy and pitch angle decreased, as expected. However, the gaps 
were consistently $\triangle_{\mathrm{r}} \approx 0.5-2 \mathrm{~cm}$ smaller with the finite gyroradius code, as illustrated for the $\mathrm{E}=1.5 \mathrm{MeV}$ case in Fig. 24. At the bottom of Fig. 28 is an example of an alpha orbit with $\mathrm{E}=2.0 \mathrm{MeV}$ and $\chi=50^{\circ}$ which just missed the first outer limiter. Such an orbit, if followed further, would also miss all the outer limiters, at least in the absence of TF ripple. The difference between the gaps calculated by the ORBIT and Lorentz codes is not due to the finite gyroradius itself, but most likely due to the slightly different radial profile of the vacuum fields or the slightly non-circular flux surfaces used in this full 2-D equilibrium model. This shows again that the structure of the vacuum fields is very important for the gap and limiter shadowing important and uncertain in these limiter shadowing calculations.

\section{Estimate of Expected Absolute Flux}

Given the strong radial dependence of the measured alpha signals, the absolute alpha flux into the detector can only be understood by using some type of limiter shadowing model. Recall that the measured alpha collection fractions for the baseline case were estimated to be in the range $10^{-7}$ to $10^{-6}$ (Fig. 11), i.e. the absolute alpha flux into the detector aperture varied from $\approx 10^{10}$ to $10^{11}$ alphas/sec over a $3 \mathrm{~cm}$ range of aperture position for a typical DT neutron rate of $\approx 10^{17} \mathrm{n} / \mathrm{sec}$.

Based on the discussion of the preceding sections, a qualitative picture of the limiter shadowing effect is shown in Fig. 29. There are three components to the expected alpha signals: the radially diffusing TF ripple loss when the gap is positive, the "confined" alphas seen by the detector only when the gap is negative (see Sec. VI.C), and the first-orbit loss to the limiter when the gap is positive. The TF ripple diffusion is dominant only in the radial region near the geometrical limiter shadow where the radial jump due to SRD is comparable to the size of the gap.

An a priori estimate of the absolute level of TF ripple loss collected by the detector aperture can be obtained from a combination of the global ripple loss, the limiter shadowing model, and the detector and geometrical parameters: 


$$
\mathrm{F}_{\text {rip }}(\mathrm{r})=\int \underset{\mathrm{E}, \mathrm{X}}{\mathrm{P}_{\text {rip }}(\mathrm{E}, \mathrm{X}) \mathrm{P}_{\mathrm{r}}(\mathrm{r}, \mathrm{E}, \mathrm{X}) \mathrm{P}_{\theta}(\mathrm{r}, \mathrm{E}, \mathrm{X}) \mathrm{P}_{\text {gyro }}(\mathrm{r}, \mathrm{E}, \mathrm{X})}
$$

Here $\mathrm{F}_{\text {rip }}(\mathrm{r})$ is the alpha ripple loss collection fraction in the detector when its aperture is at a radius " $r$ ", $\mathrm{P}_{\text {rip }}(\mathrm{E}, \mathrm{X})$ is the global probability for TF ripple loss as a function of the final alpha energy and pitch angle (e.g. taken from ORBIT calculations such as Fig. 9), $\mathrm{P}_{\mathrm{r}}(\mathrm{r}, \mathrm{E}, \mathrm{X})$ is the probability for an alpha ripple loss orbit to be lost within the radial acceptance range of the detector aperture $(\approx 0.07 \mathrm{~cm}), \mathrm{P}_{\theta}(\mathrm{r}, \mathrm{E}, \mathrm{X})$ is the probability for an alpha ripple loss orbit to be lost within the poloidal acceptance range of the detector aperture, and $\mathrm{P}_{\text {gyro }}(\mathrm{r}, \mathrm{E}, \mathrm{X})$ is the probability that an alpha ripple loss orbit entering the front aperture of the detector has a gyrophase which also passes through the back aperture of the detector (see Fig. 4). In general, each of these geometrical factors depends on the energy and pitch angle of the alphas, so the total ripple loss at a given " $r$ " is the sum over all relevant $(E, X)$.

The simplest estimate for the TF ripple loss for an aperture position just outside the limiter shadow can be made by assuming that the global ripple loss $(\approx 7 \%)$ is radially distributed uniformly over a width equal to the two SRD "jumps" on the last confined alpha orbit (perhaps $\approx 6 \mathrm{~cm}$ ), poloidally distributed uniformly over a $30^{\circ}$ angle near the outer midplane (Fig. 9), and uniformly distributed in gyrophase angle at the aperture. For these assumptions $\mathrm{P}_{\text {rip }}=7 \times 10^{-2}, \mathrm{P}_{\mathrm{r}}=(0.07 \mathrm{~cm}) /(6 \mathrm{~cm}) \approx 1 \times 10^{-2}, \mathrm{P}_{\theta}=(0.2 \mathrm{~cm}) /$ $(50 \mathrm{~cm}) \approx 4 \times 10^{-3}$, and $\mathrm{P}_{\text {gyro }} \approx(.07 \mathrm{~cm}) /(1 \mathrm{~cm}) \approx 7 \times 10^{-2}$; thus $\mathrm{F}_{\text {rip }} \approx 2 \times 10^{-7}$. This crude estimate is close to alpha collection fraction near $\mathrm{r}_{\mathrm{ap}}=-0.5 \mathrm{~cm}$ (Fig. 11), but only accurate to within an order-of-magnitude.

The most uncertain part of this estimate is the radial profile of the probability distribution of TF ripple loss in the shadow of the limiter. As discussed in Sec. C, this function depends sensitively on the alpha energy and pitch angle, as well as the magnetic field structure in the vacuum which determines the gap sizes. Even for a fixed gap size the radial profile depends not only on the average radial jump but on the distribution of jump sizes. This sensitivity is discussed in more detail in Appendix 2. 
The expected $\mathrm{TF}$ ripple loss can alternatively be estimated by comparison with the first-orbit loss, which can be calculated absolutely using the Lorentz orbit code. The calculated ripple loss near the poloidal angle of the detector is locally a factor of $\approx 3$ higher than the first-orbit loss (Fig. 9), and the radial extent of first-orbit loss behind the limiter shadow is also larger due to its effectively large "jump" at the banana tip, which causes the loss to be distributed over a larger poloidal angle. Estimating this jump to be $\approx 3$ times larger than for TF ripple loss, and assuming the same probability of gyrophase detection, the estimated ripple loss just behind the limiter shadow is $\approx 10$ times the calculated first-orbit loss into the detector, i.e. similar to the above estimate.

When the aperture is effectively inside the limiter shadow the detector can potentially collect low-pitch angle alpha orbits which would be confined except for the presence of the detector itself [11]. The probability of being detected is then the first-orbit collection efficiency multiplied by the average number of poloidal transits made before the orbit intersects the $\approx 6$ $\mathrm{cm}$ wide detector shaft (Fig. 4). This number is typically $(2 \pi \mathrm{a} / 6 \mathrm{~cm}) \approx 100$, so that the collection fraction for such "confined" alphas could be up to 100 times the first-orbit loss level, i.e. consistent with the level seen for the $r_{a p}=-$ $2 \mathrm{~cm}$ aperture position for the baseline case.

These are clearly only order-of-magnitude estimates of the absolute flux, and so are not useful for interpreting the relatively small differences between the results for different $q(r)$ seen in this experiment. A significantly better limiter shadowing model would be needed to calculate the relative alpha flux vs. detector position before these measurements can be understood quantitatively. Some potential improvements are discussed in Sec. VII.

\section{Discussion}


This experiment found significant changes in the alpha particle loss to a detector near the outer midplane depending upon the $q(r)$ profile and the alpha gyroradius in TFTR DT plasmas. However, the measured alpha loss was not simply proportional to the calculated global alpha ripple loss, as had been expected initially, but instead appears to depend sensitively on the details of the limiter shadowing process. Some features of the data were qualitatively explained by simplified modeling, but a quantitative understanding of the data has not yet been obtained. Such an understanding would require a considerably improved ripple loss model and a much better knowledge of the vacuum magnetic fields and of the energy distribution of the escaping alphas.

\section{A. Summary of Experimental Results}

Alpha particle loss near the outer midplane of TFTR was measured for three different DT plasma scans, namely a "q(r) scan", a "q(a) scan", and a " $\rho$-scan". Data was taken for each type of plasma for three different aperture positions near the geometrical shadow of the outer limiter.

The main experimental results were:

1. for all cases the DT neutron-normalized alpha loss increased as the detector aperture was moved inward toward the plasma center, typically with an e-folding length of $\approx 1 \mathrm{~cm}$ between $\mathrm{r}_{\mathrm{ap}}=+1 \mathrm{~cm}$ (outboard) and $-2 \mathrm{~cm}$ (inboard) with respect to the geometrical limiter shadow at $r_{a p}=0 \mathrm{~cm}$,

2. at a fixed plasma current and $q(a)$, the neutron-normalized alpha loss increased for a higher core $q(r)$ (i.e. broader current profile), and decreased for a lower core $q(r)$ (i.e. more peaked current profile), qualitatively as expected from the simple stochastic ripple diffusion model,

3. at a fixed plasma current the neutron-normalized alpha loss was relatively constant as the toroidal field was varied, contrary with the initial expectation based on the simple stochastic ripple diffusion model, 
4. at a fixed $q(a)$, the alpha loss decreased as the alpha gyroradius $\rho$ increased with decreasing toroidal field, contrary with the initial expectation based on the simple stochastic ripple diffusion model,

5. the detected alpha pitch angles were within the range $X \approx 54-64^{\circ}$ for all cases, corresponding to trapped alpha orbits with their banana tips in the stochastic $\mathrm{TF}$ ripple diffusion region,

6. the measured gyroradii distributions showed no significant variation with the $q(r)$ profile or aperture position, and were roughly consistent with the loss of alphas at an average energy of $\mathrm{E} \approx 2.5 \pm 1 \mathrm{MeV}$,

7. the results for the baseline plasma case changed significantly when the experiment was repeated near the end of the TFTR DT run, most likely due to small changes in the outer limiter configuration made between the two experiments.

\section{B. Status of Modeling and Interpretation}

This experiment was intended to test the dependence of the stochastic ripple diffusion coefficient on $q(r)$ and the alpha gyroradius (see Eq. 1). After the experiment was done, the expected alpha particle ripple loss was calculated using the Monte Carlo guiding center code ORBIT, including the measured $q(r)$ and alpha source profiles and alpha collisionality. These calculations were limited by available computer time to 1000 alphas for each plasma type, so that only the global alpha loss could be calculated accurately, and not the local alpha loss to the very small detector aperture.

The measured neutron-normalized alpha loss was compared with the global ripple loss calculations by normalizing the data to the model results for the baseline case common to all three plasma scans (Fig. 13). The trends in the data for the three plasma scans were not well reproduced by this modeling. The calculated global ripple loss fraction only varied between $\approx 5-8 \%$, while the measured alpha collection fraction varied by up to 
a factor of 4 between the different types of plasmas at a given aperture position.

Despite this disagreement as to the scaling of the ripple loss with $q(r)$, there were some indications that the detector was measuring TF ripple loss. An absolute calibration showed that the observed loss was at least $\approx 10$ times higher than the expected first-orbit loss, even for the outermost aperture position (Fig. 11). The measured pitch angle distributions were within the range predicted by the ORBIT code for TF ripple loss (Fig. 16), and the estimated average loss energy was close to that predicted by the code for collisional TF ripple loss. Although there was some sensitivity to the presence of locked modes (Fig. 20), there is no indication that the observed loss was caused by MHD activity or other types of low-n nonaxisymmetries.

The strong dependence of the alpha loss signals on the radial position of the detector aperture suggested that the limiter shadowing effect played a strong role in determining the alpha flux to this detector. This effect was not present in the ORBIT code, which assumed a smooth first wall. Since the number of alpha orbits which could be run from birth by the ORBIT code was too low allow a realistic 3-D model of the aperture geometry, an auxiliary limiter shadowing model was employed. The crucial elements of this model were the radial "gap" distance between the marginally confined alpha orbit and the detector aperture, and the radial "jump" of the alpha orbit per poloidal transit due to stochastic ripple diffusion.

The results of this limiter shadow modeling were found to be very sensitive to these gaps and jumps, which were mostly in the range of a few $\mathrm{cm}$ for this experiment. The gaps were found to depend on the alpha particle energy and pitch angle and on the assumed qlim and Shafranov shift of the magnetic flux surfaces near the outer midplane limiter (which were not measured). The profile of the calculated alpha flux behind the limiter shadow also depends on the detailed distribution of the jump sizes, and not just on the average jump per transit (Appendix 2). 
Despite these difficulties in quantitatively modeling the limiter shadowing effect accurately, some qualitative understanding of data was obtained. By launching alpha orbits from the detector back into the plasma, the probability of missing the limiter was found to increase with increasing pitch angle, thus explaining the shift of the measured pitch distributions to higher pitch angles for the outermost aperture positions. For aperture positions nearest to the plasma, it was found that the gaps could disappear for low energy and low pitch angle alphas, thus explaining the increased signal at low pitch angles as due a contribution from "confined" alphas, which need not have any radial diffusion. The limiter shadowing model clearly has enough flexibility to explain the radial profiles and absolute levels of the measured alpha signals, but the experimental results can not yet be quantitatively explained from the basic ripple loss physics and knowledge of the limiter and detector geometry.

The conclusion from this analysis is that the modeling is presently too uncertain to allow a quantitative explanation of the data in terms of stochastic TF ripple diffusion. Potential improvements in the experiment and modeling are discussed in Sec. D.

\section{Relationship to Other Experiments}

It is interesting to compare the difficulties encountered in the present paper with results from previous fast ion loss experiments in TFTR and other tokamaks. Techniques based on measuring confined fast ions using triton burnup, neutron production, or charge exchange have different problems $[4,5,6,8,9]$.

In TFTR, the DT alpha loss to a detector $90^{\circ}$ below the outer midplane in TFTR was successfully interpreted using the first-orbit loss model without considering the effect of limiter shadowing [13]. This was possible since the aperture was located only $\approx 1 \mathrm{~cm}$ behind the limiter shadow, and designed such that all the alpha loss orbits were unobstructed by the limiter on their first transit from the plasma to the detector. However, an anomalous low-energy alpha loss component was recently measured by a 
foil deposition detector located $\approx 1 \mathrm{~cm}$ above the limiter shadow at this poloidal location [26], and similar difficulties in interpreting the limiter shadowing effect were also encountered there.

Previous studies of DD fusion product loss at the outer midplane of TFTR also discussed the importance of the limiter shadowing effect on its interpretation [11]. The qualitative features of the DD data were similar to the DT data described here and in [12]. However, the earlier DD data was taken for aperture positions far behind the limiter shadow $\left(\mathrm{r}_{\mathrm{ap}} \approx+5-10 \mathrm{~cm}\right)$, so that a comparison could be made with the first-orbit loss. A direct measurement of the shadowing effect was also made using a movable obstacle, allowing a clearer inference of the radial diffusion rate and comparison with the stochastic diffusion model.

The only other experiments which directly measured fast ion loss due to stochastic ripple diffusion (also called banana drift diffusion) were done using an IRTV in JT-60U [5,27], where the wall heating due to ICRH minority and NBI ion loss was compared with the OFMC ripple loss code. This measurement technique averages over pitch angle, energy, and the radial coordinate, but has a better spatial coverage than the scintillator detector in TFTR. Despite the relatively smooth outer wall of JT-60U, the first measurements could not be quantitatively interpreted due to a slight misalignment of the outer wall tiles $(\approx 0.2 \mathrm{~cm})$, which caused localized heat deposition at the tile edges [5]. Later measurements were more successful in comparing the total NBI heat flux to the outer wall with the banana drift diffusion calculated by the OFMC code (similar to the ORBIT code). The spatial profile of the banana drift ripple loss could be roughly explained by the OFMC calculations (on $\approx 10 \mathrm{~cm}$ scale), but could not be explained on a smaller scale $(\approx 0.1-1 \mathrm{~cm})$.

The conclusion from this comparison with other experiments is that the stochastic ripple diffusion of fast ions to the wall has never been quantitatively understood on a scale comparable to the detector size of the TFTR experiment, mainly because the limiter shadowing effect can not be accurately calculated at that small scale. The ripple-trapped loss of fast ions appears to be somewhat better understood [28, 29], since the location of 
this loss channel is mainly determined by the shape of the TF ripple wells and of the ripple-induced wobble in the outermost magnetic field lines, and not by shadowing due to remote limiters.

\section{Potential Improvements}

The main difficulty in the analysis of the present experiment is that the escaping alpha banana orbits undergoing stochastic ripple diffusion are intercepted by the limiter near the outer midplane, and any diffusion behind this limiter has a small radial step size characteristic of the SRD. This makes the location of the alpha loss highly dependent on the limiter and detector geometry, and also on the vacuum magnetic field structure which determines the orbit shape near the outer limiter. Consequently the calculation of alpha loss to any small-area detector is quite uncertain.

The most direct way to obtain a quantitative understanding of the present experimental data would be to model the collisional ripple loss accurately enough to calculate the local flux into the detector aperture. In principle this can be done by putting the full 3-D geometry of the detector and limiters into a Monte Carlo guiding center code like ORBIT. But to be useful for interpreting the pitch angle and energy-resolved data, these code results would require at least $\approx 100$ particles to be collected by the detector. Given the detector collection efficiency of $\approx 10^{-7}$ (Fig. 11), this implies that $\approx 10^{9}$ alphas need to be followed for about one slowing down time. This would require $\approx 10^{6}$ times more computing power than has been applied to the present modeling, and so is completely impractical.

A more efficient method would be to use the ORBIT code to follow alpha orbits backward in time from the detector into the plasma all the way to their birth energy, appropriately weighting the results with the alpha source profile. This could improve the estimate of the expected limiter shadowing probability vs. energy and pitch angle, although it is not clear whether the absolute flux could be calculated this way. Since the probability of missing the limiter decreases rapidly with the number of transits (e.g. $<0.1 \%$ miss after 1000 poloidal transits when $\approx 10 \%$ miss after one poloidal 
transit), a large number of orbits $\left(\approx 10^{4}\right)$ still need to be launched at each pitch angle to simulate the detected signal [18]. It is likely that finitegyroradius effects also need to be evaluated to have confidence in the predictions of such a guiding center model.

A practical difficulty with this approach is that the results will still be sensitive to the limiter geometry and vacuum magnetic fields. For example, the uncertainty of the outer limiter radii in TFTR was at best \pm 0.3 $\mathrm{cm}$ and probably $\pm 1 \mathrm{~cm}$ by the end of TFTR operation. This introduces a significant uncertainty in the calculated ripple loss, since the radial efolding length of alpha loss in the shadow of the limiter is typically $\approx 1 \mathrm{~cm}$. The limiter shadowing effect is also very sensitive to structure of the vacuum flux surfaces and to the distribution function of SRD radial step sizes, as discussed in the Appendices. For example, $a \approx 1 \mathrm{~cm}$ uncertainty in the $\approx 20 \mathrm{~cm}$ Shafranov shift of the magnetic flux surfaces near the detector causes another $\approx 1 \mathrm{~cm}$ uncertainty in the limiter shadowing effect.

Finally, the ripple loss physics and the limiter shadowing effect also depend sensitively on the alpha energy through the shape of the banana orbits, so a precise measurement of the alpha energy spectrum is needed for a quantitative verification of the SRD mechanism. For example, such a measurement could check the expected collisional ripple loss spectrum (Fig. 9), and potentially distinguish this from "confined" alphas which should be collected with a negligible energy loss. The present instrumental uncertainty was $\pm 1 \mathrm{MeV}$, but probably needs to be $\approx 10$ times better for this purpose.

\section{Conclusion}

The conclusion from this experiment is that the alpha loss measured near the outer midplane wall of TFTR does depends on the $q(r)$ profile of the plasma. At a fixed detector aperture location the measured alpha collection fraction varied by about a factor of two with variations in the $q(r)$ profile at constant $q(a)$, and by a similar factor with variations in $q(a)$. However, the 
alpha signal also varied strongly with the detector aperture location for all cases, with a typical radial e-folding length of $\approx 1 \mathrm{~cm}$.

Guiding center code simulations alone were not able to quantitatively interpret these results in terms of the stochastic ripple diffusion model. The main difficulty was the modeling of the limiter shadowing effect, which determined the radial fall-off of the alpha flux near the outer wall. Preliminary calculations showed that the limiter shadowing effect depends on the vacuum magnetic fields near outer limiter and on the energy spectrum of the measured alpha loss, both of which determine the shape of the alpha banana orbit entering the detector. Neither of these factors was known accurately enough to quantitatively interpret the very short radial fall-off length of the alpha signal. However, the radial profile and pitch angle dependences could at least be qualitatively understood based on the shadowing of alpha orbits undergoing stochastic ripple diffusion.

For future DT tokamaks reactors such as ITER, this implies that the local alpha heat loads due to stochastic ripple diffusion to the wall can probably not be reliably predicted, even though the large-scale pattern of alpha ripple loss on the wall can be estimated from Monte Carlo codes [30,31]. This is a potential engineering problem, since overheating of even cm-scale pieces of the first wall could cause impurity influx or wall damage. The limiting factors in such calculations would be the uncertainty in the limiter shadowing effects, particularly due to slight misalignments of the first wall, and a lack of knowledge of the vacuum magnetic flux surfaces near the outer limiters.

Acknowledgments:

We thank M. Bell, R. Boivin, C.S. Chang, R.J Hawryluk, D.W. Johnson, G. LeMunyan, K.M. McGuire, D.K. Owens, J. Schivell, and M. Zarnstorff for contributions to this experiment. We especially thank V. Yavorskij of the Ukranian Institute of Nuclear Research for pointing out to us the effect of vacuum magnetic fields on alpha loss orbits in TFTR, and S. Wang of the Academia Sinica for his work on the limiter shadowing effect. This work was supported by DOE Contact DE-AC02-76-CH03073. 


\section{Appendix 1 - Sensitivity of Radial Profile to Vacuum Fields}

As pointed out by Goloborod'ko et al [32], the magnetic fields in the vacuum region outside the plasma but inside the limiter are difficult to calculate analytically. The only available solutions for TFTR come from an EFIT equilibrium reconstruction [33] which includes the MSE data on $q(\mathrm{r})$ inside the plasma and the coil currents of the $\mathrm{OH}$ and $\mathrm{EF}$ coil systems. An example of the resulting $q(\mathrm{r})$ and Shafranov shift profiles along the outer midplane for the baseline plasma case are shown in Fig. 30. A general feature of the equilibria for this experiment is that $q(r)$ increases much more rapidly in the vacuum region than would be expected from a cylindrical model in which $\mathrm{q}(\mathrm{r}) \propto(\mathrm{r} / \mathrm{a})^{2}$, and the shift becomes very negative for flux surfaces near the limiter.

These vacuum fields are important in determining the shape of the marginally confined alpha orbits in TFTR [34], and so strongly affect the size of the "gap" between the last confined orbit and the outer limiter. An example of the sensitivity of the calculated gaps to the assumed vacuum fields is shown for the baseline plasma case at the top of Fig. 31. Here the value of $q$ and the shift were kept constant at the plasma edge but varied at the limiter. For these examples the gap decreases $\approx 1 \mathrm{~cm}$ for each $1 \mathrm{~cm}$ inward shift of the flux surface at the outer wall, i.e. the gap size is uncertain by about the same amount as the shift of this flux surface. The gap also decreases as the qlim decreases, apparently since the outward orbit shift decreases with decreasing edge $\mathrm{q}$. Thus the calculated gaps have a significant uncertainty; for example, the EFIT baseline model with qlim $=25$ and shift of $-17 \mathrm{~cm}$ at the outer midplane limiter implies a gap of $5.3 \mathrm{~cm}$ for a $\mathrm{E}=2.5 \mathrm{MeV}, \chi=60^{\circ}$ alpha at $\mathrm{r}_{\mathrm{ap}}=0 \mathrm{~cm}$, whereas for a shift of $-20 \mathrm{~cm}$ the gap is $\approx 7 \mathrm{~cm}$, and for qlim=20 the gap is $\approx 4 \mathrm{~cm}$. The uncertainty of the limiter flux surface shift and $q$ is not well characterized, but is very likely to be within this range.

The sensitivity of the limiter shadowing probability to the $q$ and shift at the outer limiter is shown at the bottom of Fig. 31. As expected from the variation of the gaps to these same variables, the probability of missing the limiter (calculated as described in Sec. VI A) depends sensitively on the assumed $\mathrm{q}$ and shift at the outer limiter. For example, this probability changes from $\approx 10 \%$ for the baseline case (with qlim=25 and a shift of $-17 \mathrm{~cm}$ ) to $\approx 20 \%$ for qlim $=18$ or a shift of $-21 \mathrm{~cm}$. Again, these are likely to be within the range of uncertainty of the edge vacuum fields in TFTR. 


\section{Appendix 2 - Radial Distribution of Alphas in the Limiter Shadow}

A proper calculation of the radial distribution of TF ripple loss should take into account the full 3-D limiter and wall geometry, the escaping alpha energy and pitch angle spectrum, and the finite alpha gyroradius effects. This is well beyond present numerical modeling capabilities. However, simplified 1-D radial models for a single energy and pitch angle can be useful to illustrate the sensitivities and uncertainties in this process.

Fig. 32 show some 1-D radial models of the limiter shadowing effect. The very simplest model assumes that alphas have a constant radial jump (here $6 \mathrm{~cm}$ ), and that they all hit the limiter after one such jump. The result is a constant distribution function vs. radius in the limiter shadow, since the alphas can start their jump from anywhere in confined region at $\mathrm{r}<0$. The magnitude of the probability of detection depends on the assumed radial width of the aperture, which was taken to be $0.1 \mathrm{~cm}$ in this example. This type of assumption used to estimate the absolute flux (Sec. VI.D).

The next simplest model assumes the jump sizes have some random distribution, e.g. for SRD the vertical jump size depends on the toroidal angle of the banana tip (Eq. 4). For the purposes of illustration, the results for an assumed Gaussian jump distribution of $\mathrm{P}(\mathrm{r}) \propto \exp \left(-\mathrm{r}^{2} / \delta^{2}\right)$ with $\delta=6$ $\mathrm{cm}$ is shown in Fig. 32 . The probability of detection is $\approx 2$ times lower at $\mathrm{r}=6$ $\mathrm{cm}$ for this assumption than for a constant jump.

However, both of these models assumed that the alpha was lost to the detector after one jump in the limiter shadow, but in reality the alpha orbit has a finite probability of hitting the limiter instead of the detector on each jump [18]. A 1-D numerical model was constructed based on the assumption that alphas have a Gaussian distribution of jumps and are detected only within the aperture width of $0.1 \mathrm{~cm}$, but have a finite probability of hitting the limiter without being detected (if they landed at $r<0$ , they jumped again). Results from this model are shown in Fig. 32 for a $50 \%$ probability of hitting the limiter per jump and various Gaussian jump size $\delta$. The radial distributions are exponential with an e-folding length considerably smaller than the mean jump size, since most of the alphas are lost to the limiter before they are detected. The probability of detection varied considerably with both the jump size and the probability of hitting the limiter per jump.

To be more rigorous, the distribution of jump sizes should be based on the SRD model. A sample calculation of the time evolution of 3.5 MeV alpha banana tip locations during SRD in TFTR is shown in Fig. 33. When the banana tips start near the SRD threshold boundary their tip motion is regular and not stochastic, and some of this regularity can persist even well within the "stochastic" domain [2]. Thus for quantitative calculations it is not adequate to model the radial diffusion as a purely random process, and all the details of the orbit motion must be retained. 
$\mathrm{R}=2.52 \mathrm{~m}$

$\mathrm{a}=0.87 \mathrm{~m}$

$\mathrm{I}=1.4$ to $1.0 \mathrm{MA}$ (during NBI)

$\mathrm{B}=4.7$ to $3.4 \mathrm{~T}($ at $\mathrm{R}=2.52 \mathrm{~m})$

$\mathrm{NBI}=7.5 \mathrm{MW}(1 \mathrm{~T}$ beam and $2 \mathrm{D}$ beams $)$

$\mathrm{n}_{\mathrm{e}}(0)=3.0 \pm 0.5 \times 10^{13} \mathrm{~cm}^{-3}$

$\mathrm{T}_{\mathrm{e}}(0)=5.5 \pm 0.5 \mathrm{keV}$

$\mathrm{T}_{\mathrm{i}}(0)=10-15 \mathrm{keV}$

$\left\langle\tau_{\alpha, \mathrm{E}}\right\rangle=\approx 0.1 \mathrm{sec}$

$<\tau_{\perp, 90^{\circ}}>\approx 10 \mathrm{sec}$

$<\mathrm{Z}_{\mathrm{eff}}>\approx 3$

Table 2 - Magnetic Equilibrium Fits for Various Cases

$$
\mathrm{q}(\mathrm{r})=\mathrm{q} 0+\mathrm{q} 1(\mathrm{r} / \mathrm{a})+\mathrm{q} 2(\mathrm{r} / \mathrm{a})^{2}+\mathrm{q} 3(\mathrm{r} / \mathrm{a})^{3}
$$

$R_{0}$ and $B_{0}$ defined at magnetic axis; Sexp = source exponent

\begin{tabular}{lcccccccccc} 
Shot & $\mathrm{q} 0$ & $\mathrm{q} 1$ & $\mathrm{q} 2$ & $\mathrm{q} 3$ & $\mathrm{R}_{0}(\mathrm{~m})$ & $\mathrm{B}_{0}(\mathrm{~T})$ & $\mathrm{qcyl}_{\text {c }}(\mathrm{a})$ & $\mathrm{S}_{\exp }$ & Loss \% \\
\hline baseline & 0.857 & -1.18 & 8.85 & -2.01 & 2.65 & 4.52 & 6.52 & 7.0 & 7.5 \\
$\begin{array}{l}\text { ramp- } \\
\text { down }\end{array}$ & 0.936 & -1.87 & 6.74 & 0.708 & 2.65 & 45.2 & 5.52 & 5.5 & 5.9 \\
$\begin{array}{l}\text { ramp- } \\
\text { up }\end{array}$ & 1.29 & 2.62 & -4.46 & 7.07 & 2.65 & 45.2 & 6.52 & 7.0 & 7.1 \\
$\begin{array}{l}\text { med- } \\
\text { q(a) }\end{array}$ & 0.756 & -0.327 & 2.99 & 2.06 & 2.65 & 37.2 & 5.45 & 7.0 & 5.3 \\
$\begin{array}{l}\text { low- } \\
\text { q(a) }\end{array}$ & 0.696 & 0.547 & -0.838 & 4.35 & 2.65 & 32.6 & 4.78 & 6.0 & 5.5 \\
med $\rho$ & 0.737 & 0.449 & 1.52 & 3.98 & 2.67 & 37.0 & 6.75 & 6.5 & 6.1 \\
high $\rho$ & 0.767 & -0.086 & 1.41 & 4.84 & 2.68 & 32.2 & 6.93 & 6.0 & 6.6 \\
& & & & & & & & & &
\end{tabular}




\section{Table 3 - Shot List for $R=2.52 \mathrm{~m}$ Cases}

all $\mathrm{P}=7.5 \mathrm{MW}$ NBI from 4.0-4.8 sec in DT; neutron rate $\mathrm{S}_{\mathrm{DT}}$ averaged 4.5-4.8 sec Shot Ip (MA) $\mathrm{B}(\mathrm{T})$ $\mathrm{SDT}(\mathrm{x} 1017 \mathrm{n} / \mathrm{s})$ Probe (cm) Alpha Loss (rel)

\begin{tabular}{|c|c|c|c|c|c|}
\hline 87473 & 1.4 & 4.7 & 1.22 & -0.5 & 1.04 \\
\hline 87474 & 1.4 & 4.7 & 1.34 & -2.0 & 3.95 \\
\hline 87475 & 1.4 & 4.7 & 1.26 & +1.0 & 0.31 \\
\hline 87504 & 1.4 & 4.7 & 1.20 & -0.5 & 0.96 \\
\hline 87505 & 1.4 & 4.7 & 1.23 & -0.5 & 0.99 \\
\hline 87560 & 1.4 & 4.7 & 1.29 & -0.5 & 1.02 \\
\hline 87499 & $2.2->1.4$ & 4.7 & 1.13 & -0.5 & 0.83 \\
\hline 87500 & $2.2->1.4$ & 4.7 & 0.99 & -0.5 & 0.67 \\
\hline 87501 & $2.2->1.4$ & 4.7 & 0.98 & -0.5 & 0.63 \\
\hline 87502 & $2.2->1.4$ & 4.7 & 0.98 & -2.0 & 1.23 \\
\hline 87503 & $2.2->1.4$ & 4.7 & 0.99 & +1.0 & 0.19 \\
\hline 87511 & $2.2->1.4$ & 4.7 & 0.94 & -0.5 & 0.68 \\
\hline 87512 & $2.2->1.4$ & 4.7 & 0.74 & -0.5 & 0.80 \\
\hline 87561 & $2.2->1.4$ & 4.7 & 1.18 & -0.5 & 0.73 \\
\hline 87508 & $1.8->1.4$ & 4.7 & 1.22 & -0.5 & 0.95 \\
\hline 87509 & $1.8->1.4$ & 4.7 & 1.19 & -2.0 & 3.24 \\
\hline 87510 & $1.8->1.4$ & 4.7 & 1.22 & +1.0 & 0.31 \\
\hline 87520 & $0.6->1.4$ & 4.7 & 1.33 & -0.5 & 1.44 \\
\hline 87521 & $0.6->1.4$ & 4.7 & 1.34 & -2.0 & 4.40 \\
\hline 87522 & $0.6->1.4$ & 4.7 & 1.34 & +1.0 & 0.38 \\
\hline 87562 & $0.6->1.4$ & 4.7 & 1.38 & -0.5 & 1.53 \\
\hline 87563 & $0.6->1.4$ & 4.7 & 1.37 & -0.5 & 1.48 \\
\hline 87524 & 1.4 & 3.9 & 1.30 & -0.5 & 0.81 \\
\hline 87525 & 1.4 & 3.9 & 1.30 & -2.0 & 2.55 \\
\hline 87526 & 1.4 & 3.9 & 1.27 & +1.0 & 0.39 \\
\hline 87529 & 1.4 & 3.4 & 1.30 & -0.5 & 0.95 \\
\hline 87530 & 1.4 & 3.4 & 1.30 & -2.0 & 2.11 \\
\hline 87531 & 1.4 & 3.4 & 0.76 & +1.0 & 0.72 \\
\hline 87535 & 1.15 & 3.9 & 1.25 & -0.5 & 0.59 \\
\hline 87536 & 1.15 & 3.9 & 1.29 & -2.0 & 1.70 \\
\hline 87537 & 1.15 & 3.9 & 1.29 & +1.0 & 0.29 \\
\hline 87542 & 1.0 & 3.4 & 1.26 & -0.5 & 0.38 \\
\hline 87543 & 1.0 & 3.4 & 1.26 & -2.0 & 0.91 \\
\hline 87544 & 1.0 & 3.4 & 1.26 & +1.0 & 0.24 \\
\hline
\end{tabular}


Table 4 - Sensitivity of ORBIT Calculations (Baseline Case)

loss after 5000 transits loss after 50,000 transits
(a) standard modeling
$2.9 \%$
$6.7 \%$
(b) standard w/ lower $\delta \mathrm{E}$
$2.7 \%$
$5.4 \%$
(c) improved vacuum (1000)
$3.0 \%$
$7.1 \%$
(d) improved vacuum (2500)
$3.2 \%$
$6.6 \%$
(e) broader source profile
$2.9 \%$
(f) no ripple with collisions
$1.2 \%$
(g) no ripple no collisions
$0.2 \%$
(h) larger ripple w/collisions
$7.9 \%$

(a) standard model results shown with outer limiter $q=10$ and shift $=-5 \mathrm{~cm}$

(b) numerical accuracy increased from $\delta \mathrm{E}=5 \times 10^{-8}$ to $5 \times 10^{-9}$ per step

(c) standard case but with limiter $\mathrm{q}=25$ and shift $=-17 \mathrm{~cm}$ (1000 alphas)

(d) same as (c) but with 2500 alphas

(e) alphas source profile broadened from $\mathrm{S}_{\mathrm{exp}}=6.7$ to 5.7

(f) ripple turned off, collisions retained

(g) ripple and collisions turned off

(h) standard case with 3x normal ripple level 


\section{Figure Captions}

1. Typical time evolution of the plasmas in this experiment. At the top is the current vs. time for three shots in the $q(\mathrm{r})$ scan: one in which the plasma current was "ramped-up", one in which it was "ramped-down", and one in which it was "straight-across". The 7.5 MW of NBI was applied after the current ramps when all the plasmas were at I=1.4 MA. At the bottom is a typical time dependence of the alpha loss measured just below the outer midplane, which follow the DT neutron rate closely vs. time for this case (\#87474). This near constancy of the alpha loss per DT neutron rate during NBI was seen for all plasmas in this experiment.

2. Variation of the $q(r)$ profiles measured by MSE for the three different plasma scans in this experiment. Common to all three scans is the "baseline" shot at I=1.4 MA and B=4.7 T ("straight-across" case of Fig. 1). The " $q(\mathrm{r})$ scan" is produced by current ramping, the "q(a)" scan by varying the toroidal field at a fixed current ( $\mathrm{I}=1.4 \mathrm{MA}$ ), and the " $\rho$ scan" by varying together the I and B at a constant $q(a)$.

3. Evaluation of the Goldston-White-Boozer stochastic TF ripple diffusion criterion for $3.5 \mathrm{MeV}$ alphas for three plasmas in the $\mathrm{q}(\mathrm{a})$ scan. The curves starting from the upper left are the GWB SRD thresholds (Eq. 1) evaluated along a vertical line through the major axis for the $q(r)$ profiles of Fig. 2. The curves ending at the upper right are the TFTR ripple strengths evaluated vertically through the major axis and horizontally along $\mathrm{z}=0$. The crossing points are the radii outside of which the banana tips are in the

$\mathrm{SRD}$ region. The SRD threshold moves radially $\approx 10-15 \mathrm{~cm}$ with this variation in $q(r)$.

4. The midplane lost alpha detector has a small aperture $3 \mathrm{~cm}$ from the probe tip, oriented so as to collect alpha loss within a range of pitch angles $X \approx 40-85^{\circ}$ with respect to the co-B direction and a range of gyroradii $\rho \approx 2-12$ $\mathrm{cm}$ (see Sec. III for definitions of $\chi$ and $\rho$ ). Alphas are separated by the pinhole/slit aperture pair and strike the light-emitting scintillator plane. At the bottom is a typical scintillator image with its contours linear in light intensity. The total alpha loss is integrated over the shaded area, and the $(X, \rho)$ distributions are interpreted using the grid shown.

5. Geometry of the midplane lost alpha detector with respect to the first wall and limiters in TFTR. The detector is located $20^{\circ}$ below the outer midplane and can be moved radially between shots. The limiters are located at various toroidal angles, all with centers at $R_{l i m}=260.6 \mathrm{~cm}$ and minor radii of alim $=99 \mathrm{~cm}$. The limiter nearest to the detector covers only poloidal angles $\geq 30^{\circ}$ away from the outer midplane, but the "RF limiters" cover $\pm 30^{\circ}$ around the outer midplane. The detector aperture is scanned near the geometrical shadow formed by these limiters. 
6. Plasma equilibrium for the baseline I=1.4 MA case, showing the location of the magnetic axis and flux surfaces inside the plasma. The boundary outside of which banana tips are ripple trapped is also indicated (ripple trapping contributes a negligible fraction to the alpha ripple loss).

7. A typical 3.5 MeV alpha particle orbit undergoing TF ripple diffusion and ultimately lost to the alpha detector for the baseline I=1.4 MA case. This orbit had a pitch angle of $69^{\circ}$ and was launched "backwards in time" from an aperture location of $\mathrm{r}_{\mathrm{ap}}=-2 \mathrm{~cm}$. The banana tips were in a relatively high ripple region, and so their vertical displacements due to SRD were relatively large. This orbit was calculated using the EFIT-based vacuum fields.

8. Maps of the alpha particle ripple loss domain for the baseline I=1.4 M A case, based on ORBIT code runs of 75,000 transits (one energy e-folding). In both maps the first-orbit alpha loss region is shaded, the confined alpha region is white, the alpha ripple loss region is marked by " $\mathrm{X}$ "s, and the ripple trapping region is marked by "P"s. The top map shows that the ripple loss occurs for alphas born with pitch angles either co (+) or ctr (-) near the first-orbit loss boundary. The bottom map shows that alpha orbits born with banana tips outside a circle of radius $\approx 20 \mathrm{~cm}$ near the magnetic axis are ripple lost. However, after only 1000 transits the radius of the confined alpha region is $\approx 30 \mathrm{~cm}$, which is similar to the prediction of the collisionless GWB SRD model (see Fig. 3).

9. Results from the Monte Carlo ORBIT code modeling of the baseline case using 1000 alphas followed for one energy e-folding time. The ripple loss is concentrated within a poloidal angle of $\approx 30^{\circ}$ below the outer midplane, as expected. The middle graph shows that the pitch angle distribution of ripple loss is peaked at $\approx 60^{\circ}$ with respect to the total magnetic field. At the bottom is shown the energy spectrum of the alpha ripple loss, which has an average energy of $\approx 2.5-3.0 \mathrm{MeV}$ due to delayed collisional loss. These results are similar to previous modeling of TFTR ripple loss, and are insensitive to the choice of vacuum fields.

10. Examples of the scintillator light emission patterns for the baseline plasma case for probe aperture positions $\mathrm{r}_{\mathrm{ap}}=+1.0 \mathrm{~cm}$ and $-2.0 \mathrm{~cm}$. On the left is the raw data showing the alpha signal peaks, and on the right are the same intensity distributions unfolded in terms of the inferred pitch angle and gyroradius. The neutron and gamma-induced fiberoptic light background visible for $\mathrm{r}_{\mathrm{ap}}=+1.0 \mathrm{~cm}$ case, showing that the neutronnormalized alpha loss signal is significantly smaller for this case.

11. Alpha collection fraction, i.e. alpha flux measured by the midplane detector divided by the global DT neutron rate, as a function of the aperture position for the baseline case. The absolute value of the alpha loss was obtained by comparing the midplane signals with the $90^{\circ}$ detector signals, assuming the latter were due to first-orbit loss [13]. The alpha collection fractions were much larger than the first-orbit loss calculated for the 
midplane detector, and decreased with an e-folding length of $\approx 1 \mathrm{~cm}$ over the range of aperture positions used in this experiment.

12. Variations of the midplane alpha loss for the various plasma scans and aperture positions. For these plots the baseline case with $r_{a p}=-0.5 \mathrm{~cm}$ is normalized to unity. Two general trends are for the alpha loss to decrease with a more peaked current profile, i.e. high central $q(\mathrm{r})$, and for the alpha loss to decrease with decreasing $B$ at constant $q(a)$. All data is averaged over the steady-state period 4.5-4.8 sec during NBI.

13. Neutron-normalized midplane alpha loss for the various plasma scans, compared with the total TF ripple loss of alphas as calculated by the ORBIT guiding center code (the data points are the same as for Fig. 12). The data is normalized to the baseline ORBIT calculation for each probe position, and the lines are solely to guide the eye. The measured alpha loss varies by a factor of three for the $r_{a p}=-0.5 \mathrm{~cm}$ case, and somewhat more (less) for the $\mathrm{r}_{\mathrm{ap}}=-2.0 \mathrm{~cm}(+1.0 \mathrm{~cm})$ cases (the off-scale point may have been affected by a locked mode). There is appears to be no clear correlation between the measured variations and the calculated ORBIT code results, either for the full collisional slowing-down time (closed circles) or for the "collisionless" result evaluated after only 5000 transits.

14. Examples of pitch angle distributions of the midplane alpha loss measured in this experiment. At the top is the variation with aperture position for the baseline case, and at the bottom is the variation with plasma type for $\mathrm{r}_{\mathrm{ap}}=-2.0 \mathrm{~cm}$ (the relative amplitudes for each signal are also shown). These pitch angles are measured with respect to the toroidal field direction, and have a systematic uncertainty of about $\pm 3^{\circ}$.

15. At the top are calculated pitch angle distributions for TF ripple loss to the wall, based on ORBIT code modeling for two different plasma cases. For these (and all other plasmas in this experiment) the expected range of pitch angles for TF ripple loss is $X \approx 54^{\circ}-68^{\circ}$ with respect to the total magnetic field at the wall. At the bottom are examples of two $\mathrm{E}=2.5 \mathrm{MeV}$ alpha orbits entering the detector for the extremes of pitch angle in this range. These examples are calculated for the baseline case using the EFIT-based vacuum fields (without TF ripple).

16. Comparison between the peak pitch angles in the data and the expected range of pitch angles for TF ripple loss based on the ORBIT code. The data all fall within the expected range $\left(1^{\circ}\right.$ has been added to the data to correct for the angle between the total and toroidal field at the wall), but the model does not predict the systematic variation of the measured pitch angles vs. aperture radius. All data is averaged over the steady-state period 4.5-4.8 sec during NBI.

17. Examples of the gyroradius distributions of the midplane alpha loss measured in this experiment. At the top are the three plasmas in the $q(r)$ 
scan, all at $\mathrm{B}=4.7 \mathrm{~T}$ (normalized to each other vertically), along with model distributions for various assumed alpha energies. At the bottom are the three plasmas for the $q(a)$ scan in which the B was varied, showing an increase in the alpha gyroradius with decreasing $\mathrm{B}$.

18. Comparison between the peak of the measured gyroradius distributions with the peak locations expected for alpha loss of various energies. At full toroidal field all cases have very nearly the same peak gyroradius, which by comparison with the models implies a mean alpha loss energy of $\mathrm{E}=2.5 \pm 1$ $\mathrm{MeV}$. At lower fields the inferred average energy appears to be somewhat lower, but the uncertainties are also larger. All data is averaged over the steady-state period 4.5-4.8 sec during NBI.

19. Time dependence of the 2-D scintillator patterns for a baseline plasma case (top), and peak gyroradius vs. time (bottom). During the time of NBI there is no significant change in the observed gyroradius distribution of the alpha loss. However, after the end of NBI there is sometimes a small downward shift in the alpha loss energy, which is at least qualitatively consistent with the delayed loss expected from collisional TF ripple diffusion.

20. Effect of a stationary magnetic perturbation (SMP) on the pitch angle distribution of the midplane alpha loss. These two discharges are externally identical current ramp-down cases with the aperture at $r_{a p}=-0.5$ $\mathrm{cm}$. The case with the SMP had $\approx 20 \%$ lower total alpha loss than the case without the SMP.

21. Characteristics of the alpha loss measured in $\mathrm{R}=2.45 \mathrm{~m}$ plasmas with the same $\mathrm{B}$ and $\mathrm{I}$ as for the $\mathrm{R}=2.52 \mathrm{~m}$ baseline case. The detected alpha loss per neutron increases as the aperture is moved in to $r_{a p} \approx-5 \mathrm{~cm}$. At $r_{a p} \approx-0.5$ $\mathrm{cm}$ the alpha loss per neutron is nearly the same as for the $\mathrm{R}=2.52 \mathrm{~m}$ case, but the radial profile is different. The pitch angle distribution is peaked toward the lower end of the range which was found for $R=2.52 \mathrm{~m}$ plasmas.

22. Alpha measurements made during an extended radial probe scan done near the end of the TFTR DT run. The plasma conditions were nearly identical to those for the baseline case, but the limiter conditions were probably different due to a small radial movement of the RF antennas. The radial profile and pitch angle distribution of the alpha loss were considerably different than for the baseline case described in Figs. 11 and 14.

23. Illustration of the limiter shadowing effect for alpha orbits entering the detector aperture near the outer midplane. In the absence of TF ripple diffusion orbits, which are launched backwards in time from the detector intersect the geometrical shadow of the limiter within one poloidal transit. The confined orbits of the same energy and pitch angle are separated from the detector aperture by a radial "gap" over which alphas must jump in 
order to be detected. The expected radial profile of ripple loss and first-orbit loss is shown schematically at the bottom.

24. The calculated radial "gaps" between the marginally confined alpha orbits and the geometrical shadow of the outer limiter at the detector location, as a function of the alpha energy and pitch angle. The gaps are smaller for lower energy and lower pitch angle alphas since the shift of the orbit from the flux surfaces is smaller. The average radial "jumps" per transit due to stochastic TF ripple diffusion are also shown, both from numerical calculations using the ORBIT code and from the GWB SRD formula. The gaps for $\mathrm{E}=1.5 \mathrm{MeV}$ calculated using a more accurate vacuum model in the Lorentz orbit code are also shown for comparison.

25. Plot of the calculated probability that an alpha orbit launched from the detector aperture misses the outer limiter on its first poloidal transit. The top shows that this probability increases with pitch angle, since the jumps are larger for orbits with banana tips in a higher ripple region (see Fig. 15). The bottom shows how the probability of missing the limiter increases as the aperture is moved inward to narrow the gap. For alphas of low pitch angle, this increase is occurs only when the gap is zero, since the diffusive jump size is very small for banana tips near the stochastic threshold.

26. Comparison of the calculated pitch angle distributions from a modified "ORBIT+shadowing" model with the data for the baseline case. The peak in the modified model pitch distribution agrees well with the data for $r_{a p}=-$ $0.5 \mathrm{~cm}$, but not for $\mathrm{r}_{\mathrm{ap}}=-2.0 \mathrm{~cm}$. The model pitch distribution is similar at $r_{a p}=-0.5 \mathrm{~cm}$ for alphas in the range $\mathrm{E}=1.5-3.5 \mathrm{MeV}$, but for $\mathrm{r}_{\mathrm{ap}}=-2 \mathrm{~cm}$ there can be a contribution from "confined" alphas at low pitch angle (not shown). The relative vertical scale between the data and the models is arbitrary, and $1^{\circ}$ was added to the pitch of the data to correct for the angle between the total magnetic field and the toroidal field.

27. Radial profiles of the data for the baseline case resolved into two separate pitch angle components (top and bottom), along with limiter shadowing models for each. At high pitch angles $\left(X \approx 64 \pm 6^{\circ}\right)$, the radial profile of the data is similar to the calculations of the ripple diffusion behind the limiter shadow for $\mathrm{E}=2.5-3.5 \mathrm{MeV}$. For low pitch angles $\left(X \approx 64 \pm 6^{\circ}\right)$, the radial profile of the data is consistent with the collection of "confined" alphas when the gap is effectively zero. For the vacuum model in this calculation, this zero-gap condition occurs at an aperture radius of $r_{a p}=-4$ $\mathrm{cm}$ for $\mathrm{E}=2.5 \mathrm{MeV}$, while the data suggests this actually occurs at $r_{\mathrm{ap}}=-2$ $\mathrm{cm}$. This difference is probably due to uncertainties in the Shafranov shift of the flux surfaces in the vacuum region near the outer limiter.

28. Alpha orbit trajectories calculated backward in time from the detector at $r_{a p}=-2 \mathrm{~cm}$ toward the limiter using a finite-gyroradius Lorentz orbit code. This code has a more exact representation of the vacuum fields than the ORBIT code, but does not include TF ripple fields. The orbit with $\mathrm{E}=2.5 \mathrm{MeV}$ 
and $\chi=60^{\circ}$ hits the limiter near the outer midplane as expected, but the orbit at $\mathrm{E}=2.0 \mathrm{MeV}$ and $\chi=50^{\circ}$ misses the limiter. The latter is a "confined" alpha which can be collected by the detector when the aperture is far enough inward, even without any radial diffusion of the orbit.

29. Qualitative picture of the effects of limiter shadowing on the alpha detection in this experiment. The TF ripple loss falls off in the limiter shadow with an e-folding length comparable to the radial step size for SRD. When the aperture is far enough inward it begins to collect "confined" alphas, which need not have any radial diffusion to be detected. The measured pitch angle distributions represent a combination of these two mechanisms, since the aperture was not far enough outward in this experiment for a significant first-orbit loss component.

30. The $q(R)$ and Shafranov shift profile from the EFIT solution to the vacuum fields along the outer midplane. The vacuum $q(r)$ is considerably higher than for a cylindrical model, and the inward shifts of the vacuum flux surfaces are relatively large.

31. The top shows the sensitivity of the size of the gap for alphas of $E=2.5$ $\mathrm{MeV}$ and $X=60^{\circ}$ vs. the assumed $\mathrm{q}$ and shift at the outer limiter. The gap is particularly sensitive to the assumed shift of the flux surface at the outer limiter. The bottom shows the probability for a typical alpha orbit to miss the limiter as a function of the assumed $q$ and shift at the outer limiter. The limiter shadowing probability is also sensitive to the assumed vacuum fields.

32. 1-D models of the radial profile of alpha detection in the limiter shadow. The simplest model assumes a constant jump size and results in a constant probability of detection over this jump. If the jumps are assumed to have a Gaussian distribution the radial profile is considerably different. If the probability of hitting the limiter is non-zero at each jump, the radial profile depends on both the jump size and on this probability.

33. Sample calculation of the time evolution of the vertical banana tip position for $3.5 \mathrm{MeV}$ alpha orbits diffusing due to TF ripple in TFTR. The orbits which are born with banana tips inside at $\mathrm{r} \approx 25 \mathrm{~cm}$ do not diffuse, but just oscillate radially. The orbits which are born inside the stochastic region retain some regularity even fairly far out toward the wall. The numerical results for no ripple $(\delta=0)$ are shown to indicate the numerical accuracy of the code. 


\section{References}

1) R.J. Goldston, R.B. White, and A. Boozer, Phys. Rev. Lett. 47, 647 (1981)

2) R.B. White, R.J. Goldston, M. H. Redi, and R.V. Budny, Phys. Plasmas 3 (8) 3043 (1996)

3) M. Redi, S.H. Batha, R.V. Budny, et al, Proc. EPS Meeting, June 1997

4) W.W. Heidbrink and G. Sadler, Nucl. Fusion 34, 535 (1994)

5) K. Tobita, K. Tani, Y. Kusama, T. Nishitani, Y. Ikeda, Y. Netatani, S.V. Konovalov, M. Kikuchi, Y. Kiode, K. Hamamatsu, H. Takeuchi, and T. Fujii, Nucl. Fusion 35, 1585 (1995)

6) G. Sadler, J.M. Adams, F.E. Cecil, G.A. Cottrell, A. Gondhalekar, J.A. Hoekzema, O.N. Jarvis, M.J. Loughlin, F.B. Marcus, P. Van Belle, and M. Von Hellerman, Nucl. Fusion 35, 1609 (1995)

7) V. Basiuk, A. Becoulet, C. Grisolia, T. Hutter, G. Mayaux, G. Martin, B. Saoutic, and S. Vartanian, Nucl. Fusion 35, 1593 (1995)

8) H.H. Duong, R.K. Fisher, S.S. Medley, M.P. Petrov, N.N. Gorelenkov, R.V. Budny, D.K. Mansfield, J. M. McChesney, P.B. Parks, A.L.

Roquemore, R.B. White and S.J. Zweben, Nucl. Fusion 37, 271 (1997)

9) K. Tobita et al, 16th IAEA Fusion Energy Conference, Montreal, Canada, IAEA-CN-64/A5-6 (1996)

10) R. Boivin and S.J. Zweben, and R.B. White, Nucl. Fus. 33, 449 (1993)

11) R. Boivin and S.J. Zweben, Phys. Fluids B 5, 1559 (1993)

12) S.J. Zweben, D. S. Darrow, H.W. Herrmann, M.H. Redi, J. Schivell, R.B. White, Nucl Fusion 35, 1445 (1995)

13) S.J. Zweben, D.S. Darrow, H.W. Herrmann, S.H. Batha, R.V. Budny, C.S. Chang, Z. Chang, E.D. Fredrickson, D.L. Jassby, L.C. Johnson, F. Levinton, H.E. Mynick, D.K. Owens, J. Schivell, S.D. Scott, M.H. Redi, J.D. Strachan, K. Tobita, and K.M. Young, Nucl. Fusion 35, 893 (1995)

14) S. Batha, et al, Nucl. Fusion 35, 1465 (1995); S. Hirshman et al, Phys. Plasmas 1, 2277 (1994)

15) M.H. Redi, M.C. Zarnstorff, R.B. White, R.V. Budny, A.C. Janos, D.K. Owens, J.F. Schivell, S. D. Scott, and S.J. Zweben, Nucl. Fusion 35, 1191 (1995). 
16) D.S. Darrow, H. W. Herrmann, D. W. Johnson, R. J. Marsala, R. W. Palladino, and S. J. Zweben, Rev. Sci. Inst. 66, 476 (1995)

17) R.L. Boivin, S. Kilpatrick, D. Manos, and S. J. Zweben, Rev. Sci. Inst. 63, $4562(1992)$

18) S. Wang and S.J. Zweben, "Model of Alpha Particle Diffusion in the Outer Limiter Shadow of TFTR", PPPL-3186 (1996)

19) M. H. Redi, R.V. Budny, D. S. Darrow, H.H. Duong, R.K. Fisher, A.C. Janos, J.M. McChesney, D.C. McCune, S.S. Medley, M.P. Petrov, J.F. Schivell, S.D. Scott, R.B. White, M.C. Zarnstorff, and S.J. Zweben, Nucl. Fusion 35, 1509 (1995)

20) S. von Goeler et al, Rev. Sci. Inst. 68, 548 (1997)

21) A.T. Ramsey et al, Rev. Sci. Inst. 68, 632 (1997)

22) S.J. Zweben et al, Phys. Plasmas 1 (5), 1469 (1994)

23) H. Takahashi, Fusion Engineering and Design 34-35, 89(1997)

24) M. Tuszweski and S.J. Zweben, Rev. Sci. Inst. 63, 4542 (1992)

25) J. Felt et al, Rev. Sci. Inst. 61, 3262 (1990)

26) H.W. Herrmann et al, Nucl. Fusion 37, 293 (1996)

27) Y. Ikeda et al, Nucl. Fusion 36, 759 (1996)

28) K. Tobita et al, Phys. Rev. Lett. 69, 3060 (1992)

29) M. Tuszewski and J.P. Roubin, Nucl. Fusion 28, 499 (1988)

30) K. Tobita et al, Nucl. Fusion 35, 1585 (1995)

31) C.Z. Cheng et al, 16th IAEA Fusion Energy Conference, Montreal, Canada 1996, paper IAEA-CN-64/FP-23

32) V. Goloborod'ko, et al, Nucl. Fusion 35, 1523 (1995)

33) L.L. Lau et al, Nucl. Fusion 25, 1611 (1985)

34) V. Yavorskij, private communication (1996) 

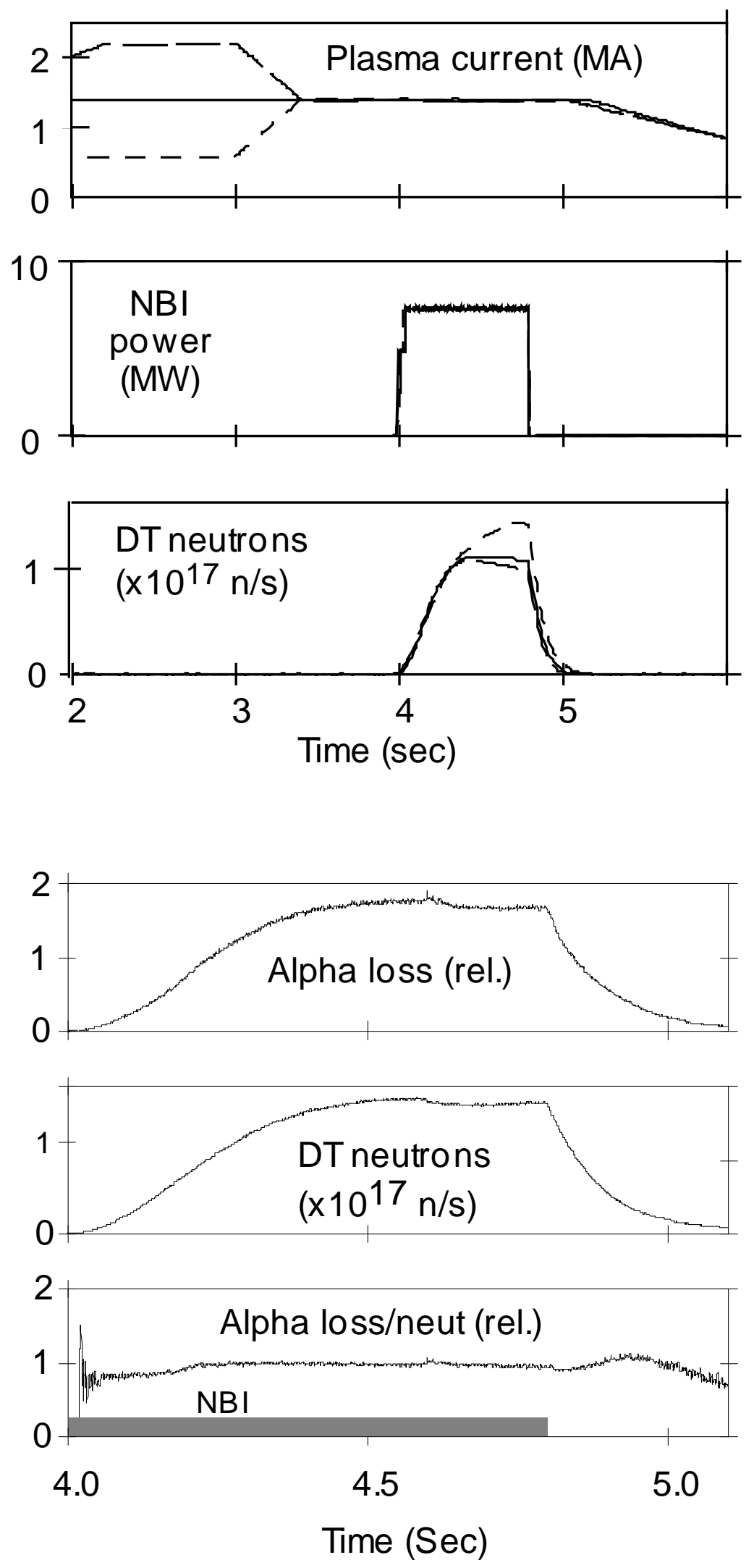

Figure 1 

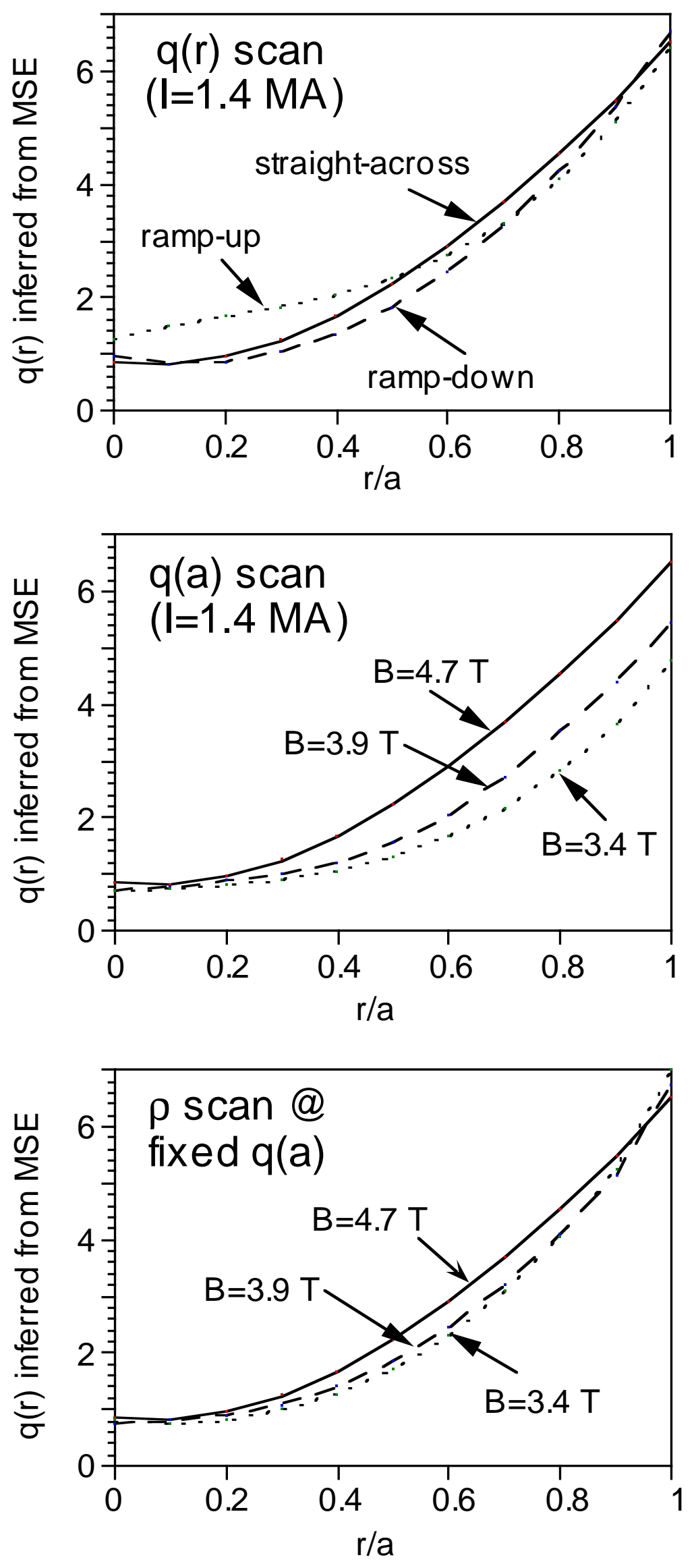

Figure 2 


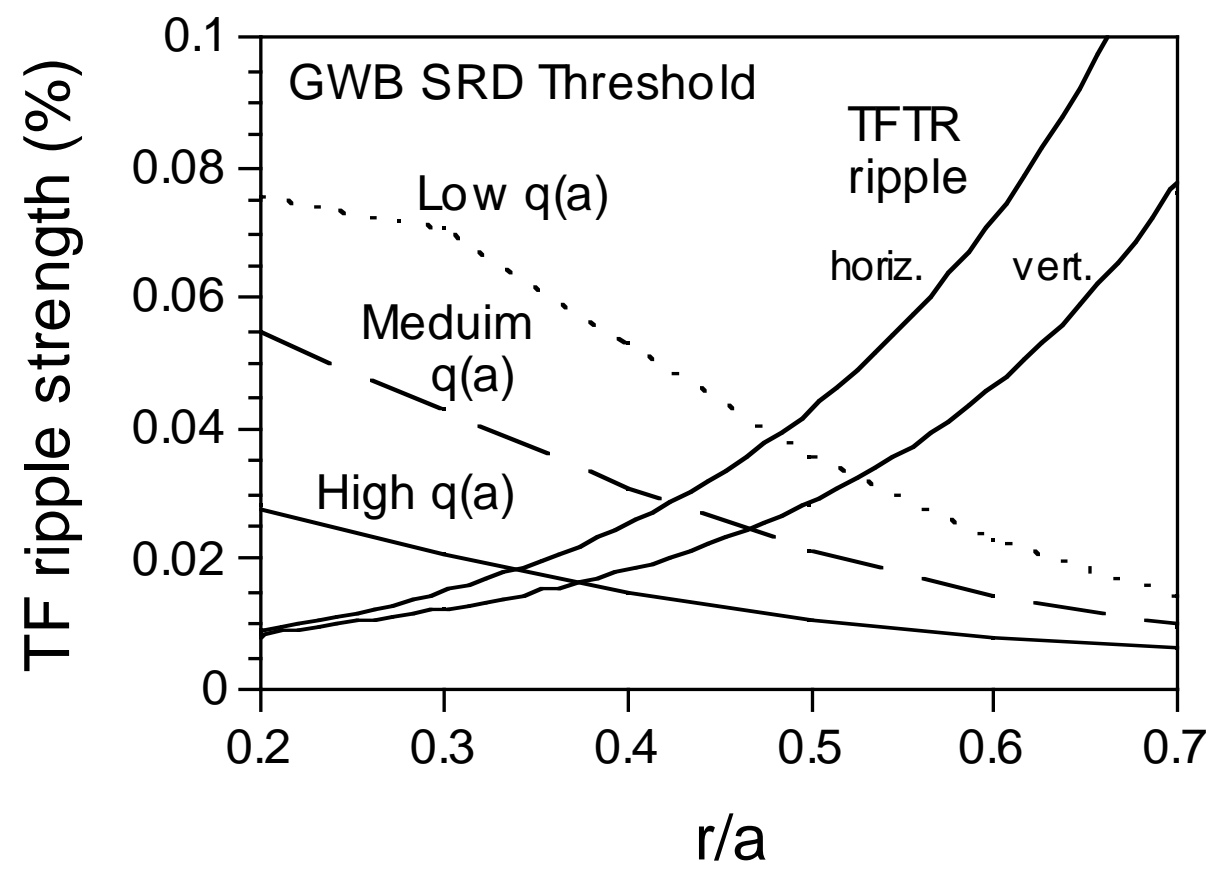

Figure 3 

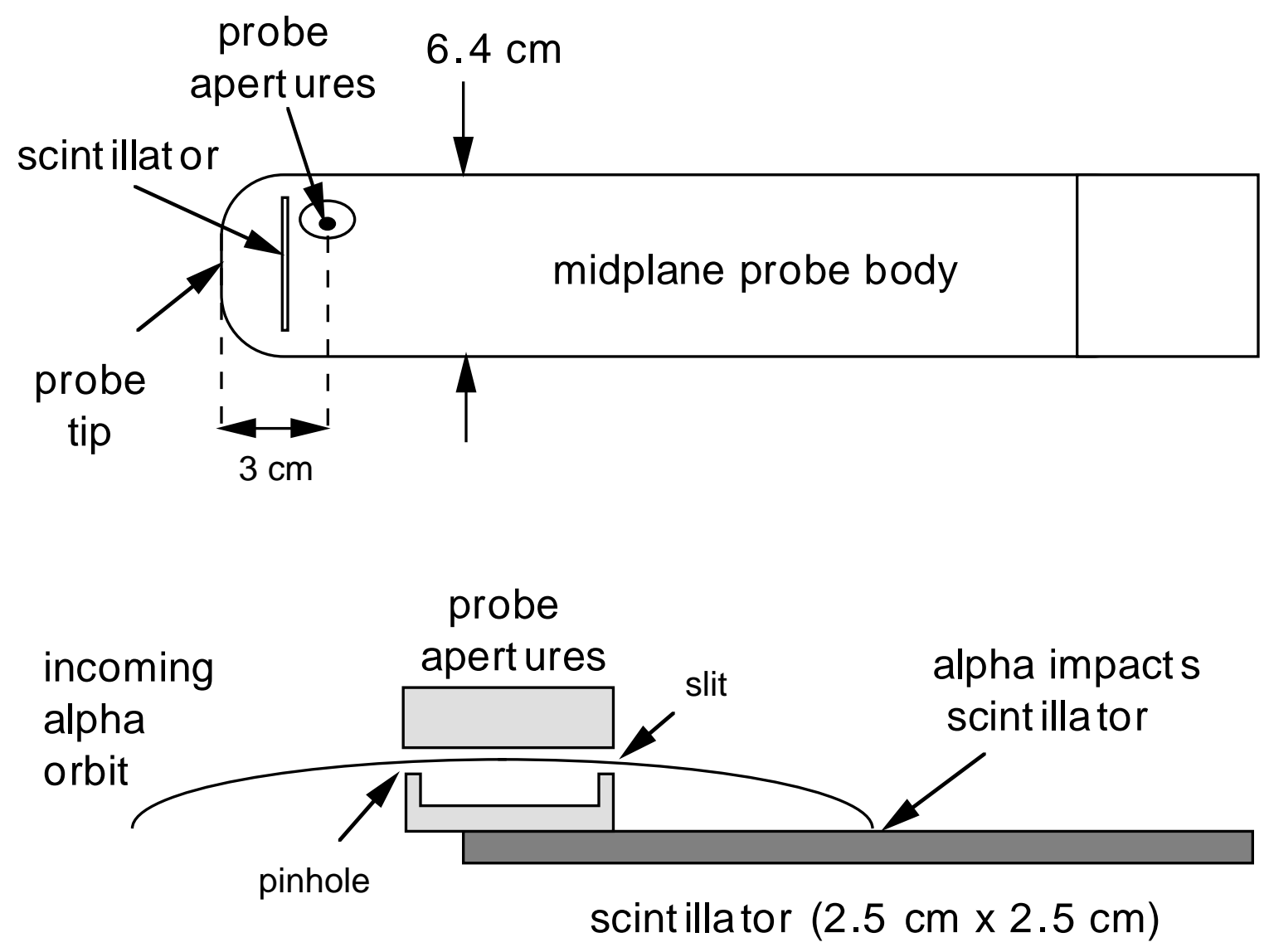

fiberbundle scint illator out line ou tline

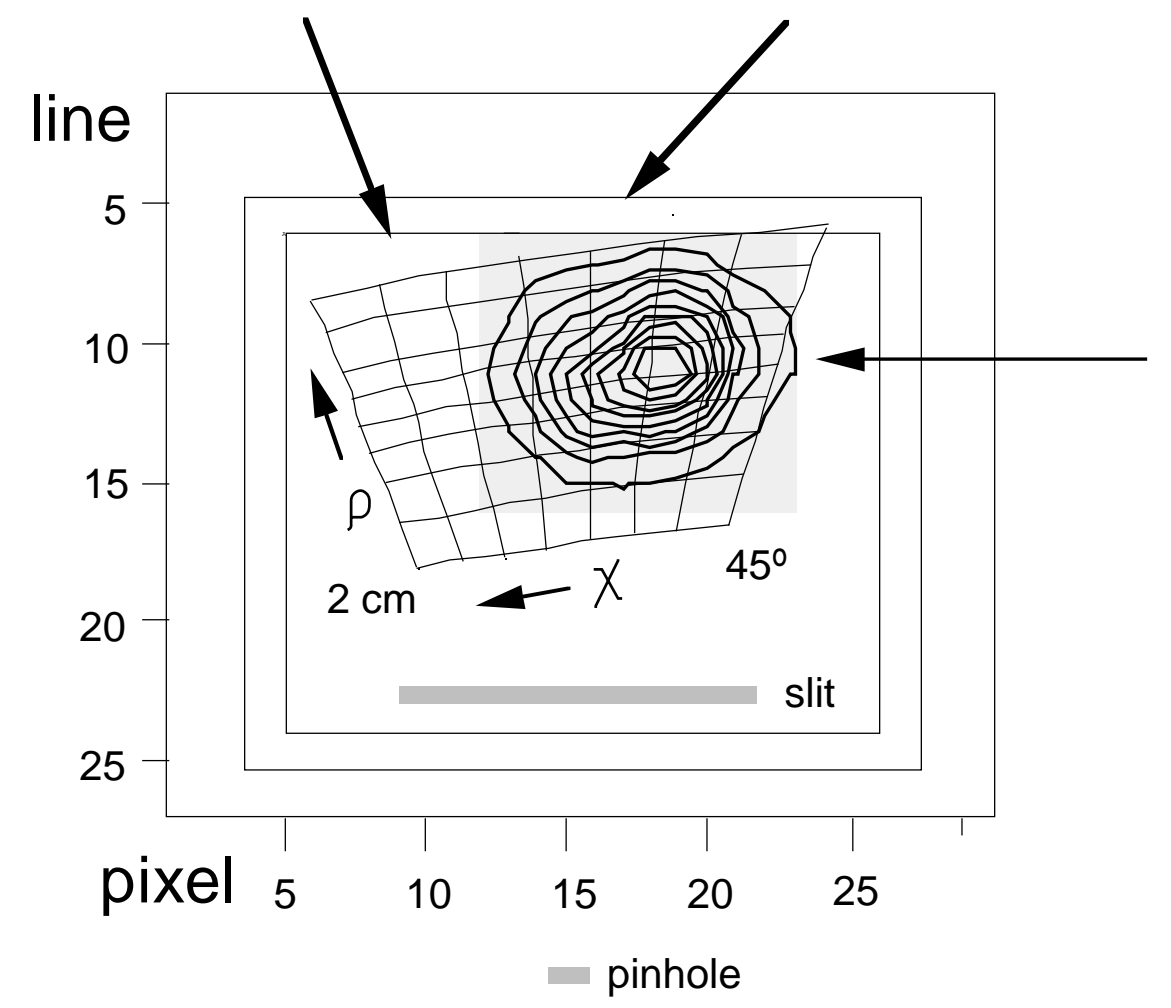

alpha signal

Figure 4 

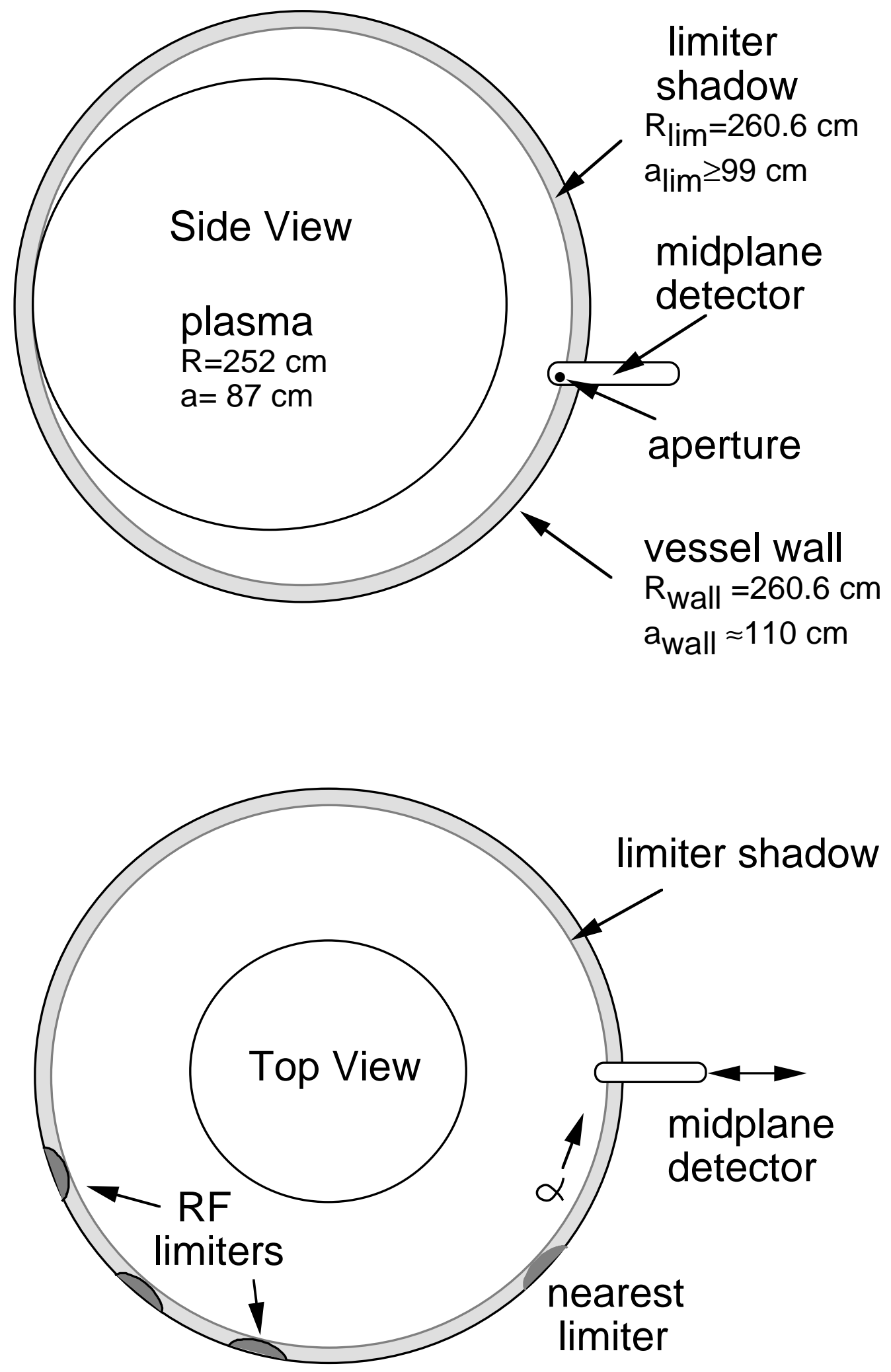

Figure 5 


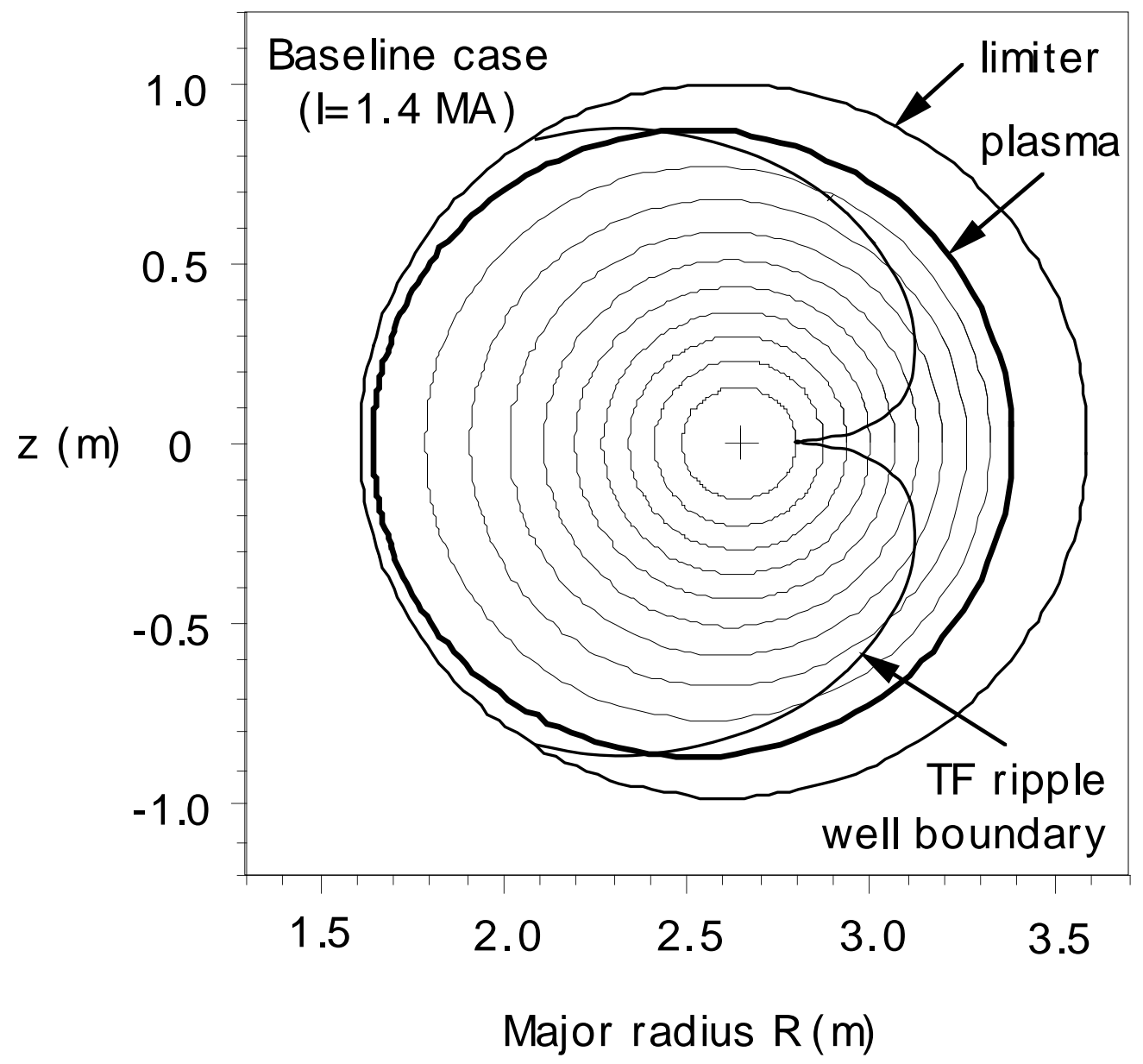

Figure 6 


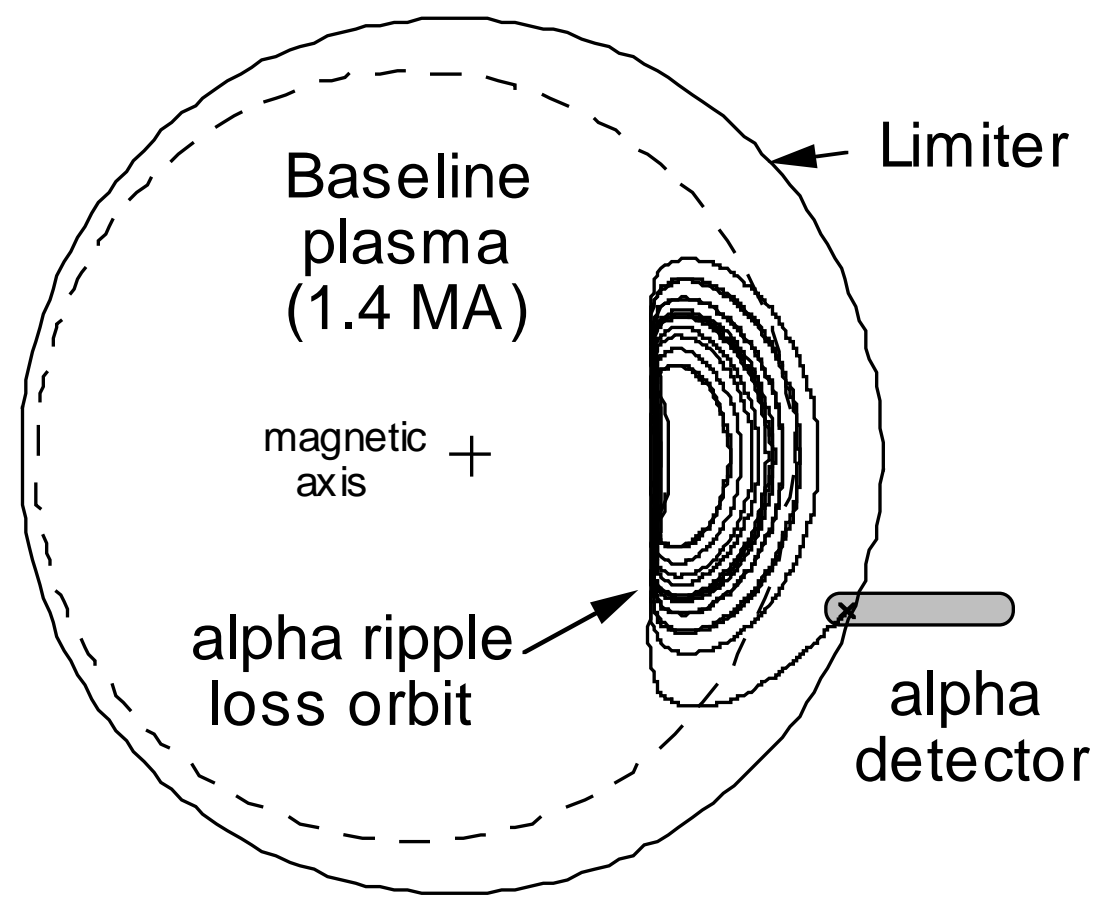

Figure 7 

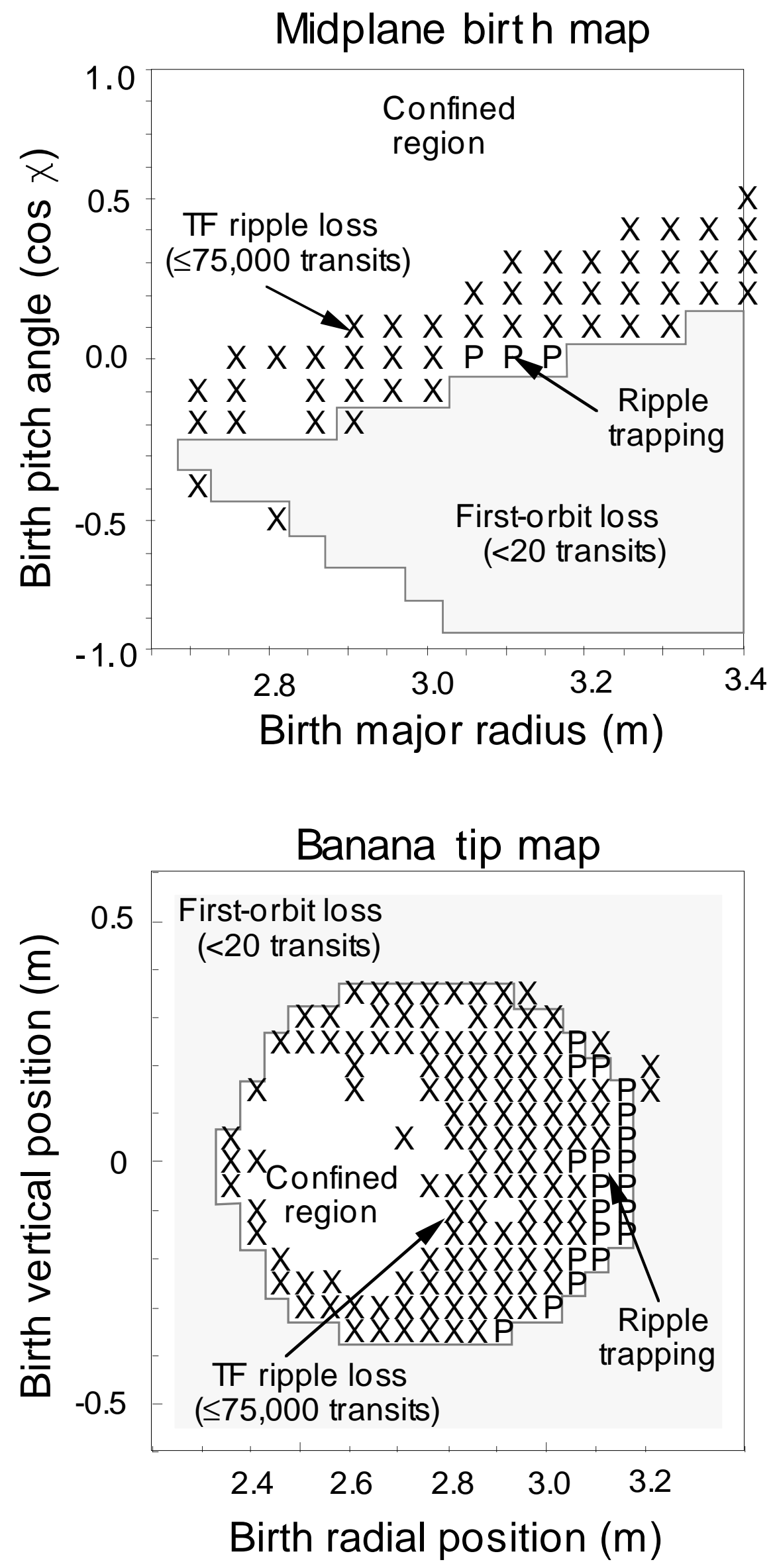

Figure 8 

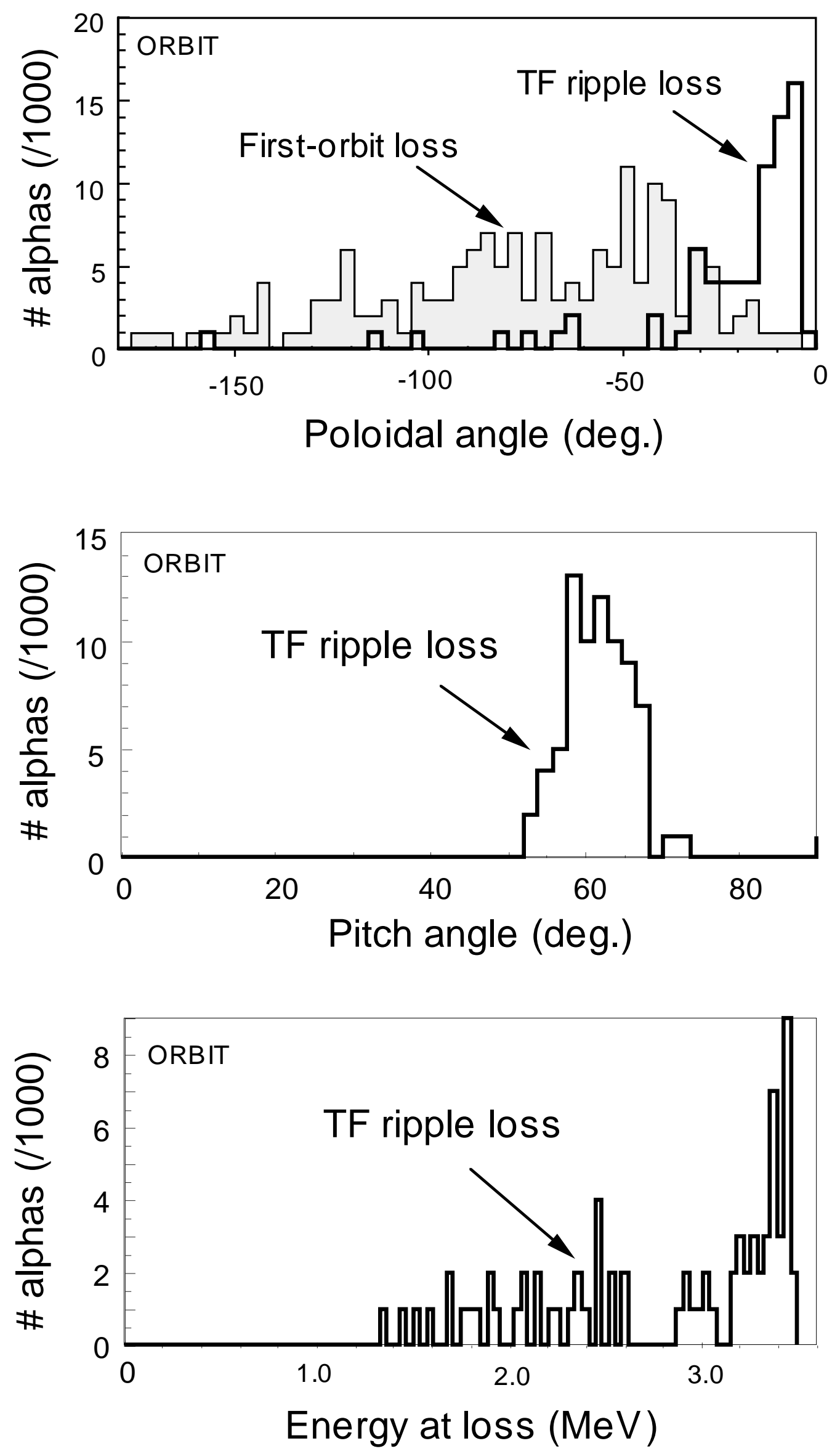

Figure 9 
Aperture location $\mathrm{r}_{\text {ap }}=+1.0 \mathrm{~cm}(\# 87475)$
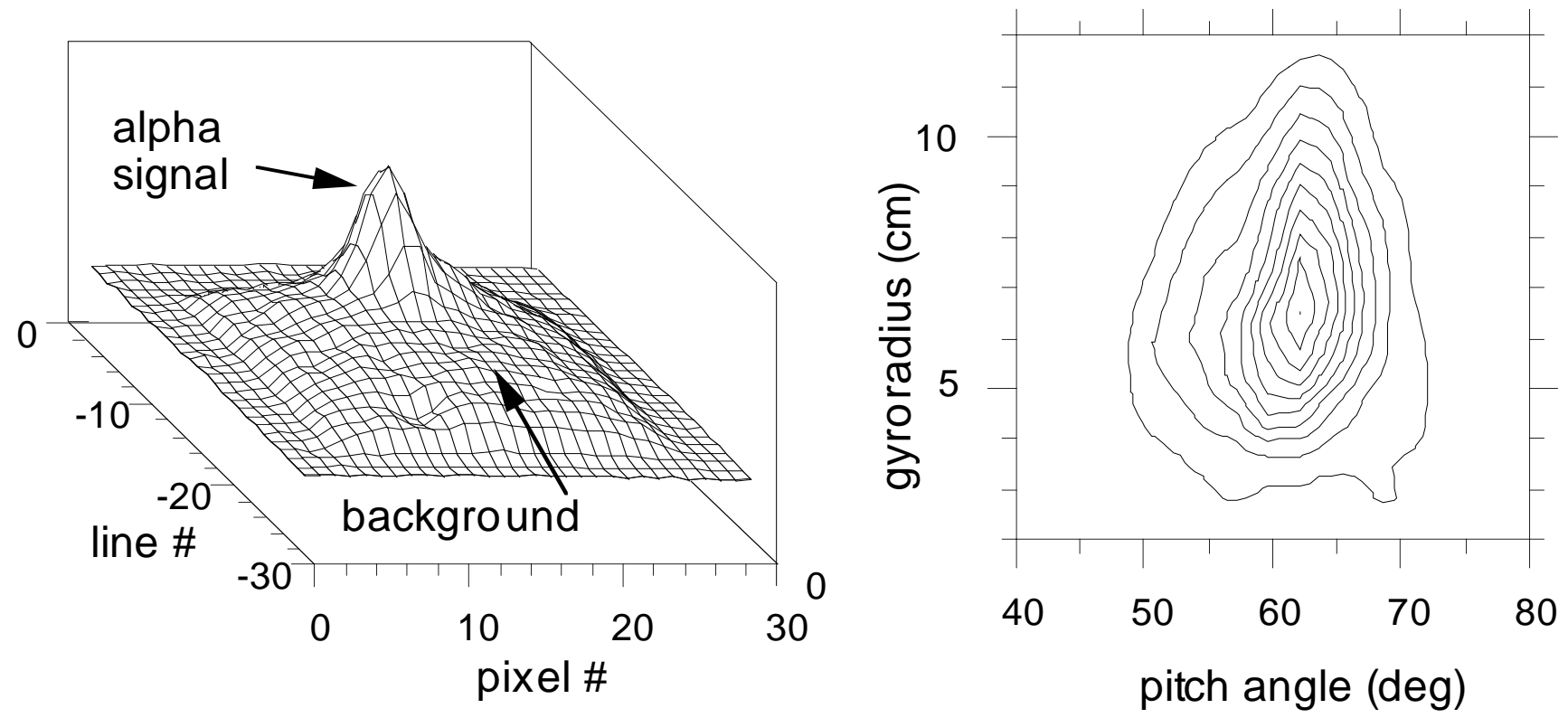

Aperture location $r_{a p}=-2.0 \mathrm{~cm}(\# 87474)$
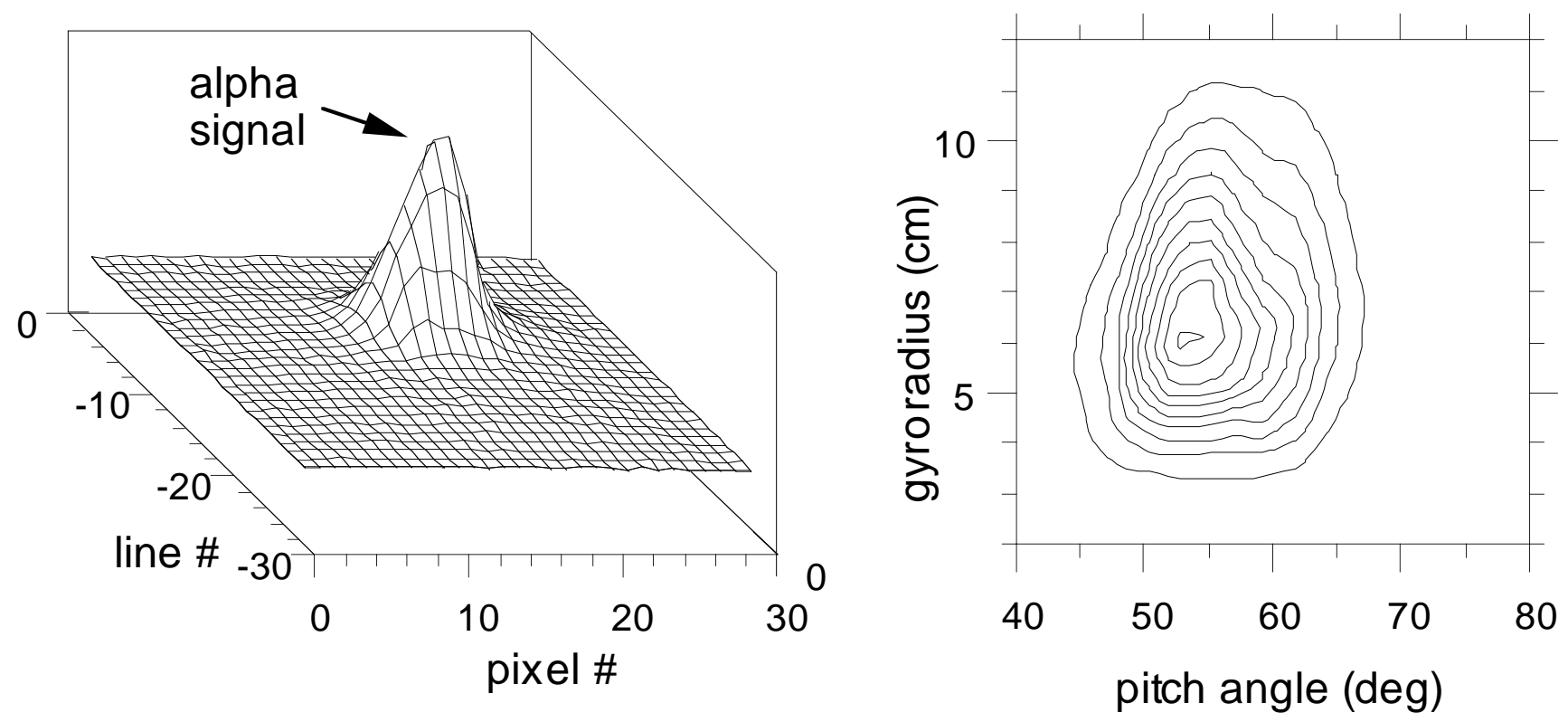

Figure 10 


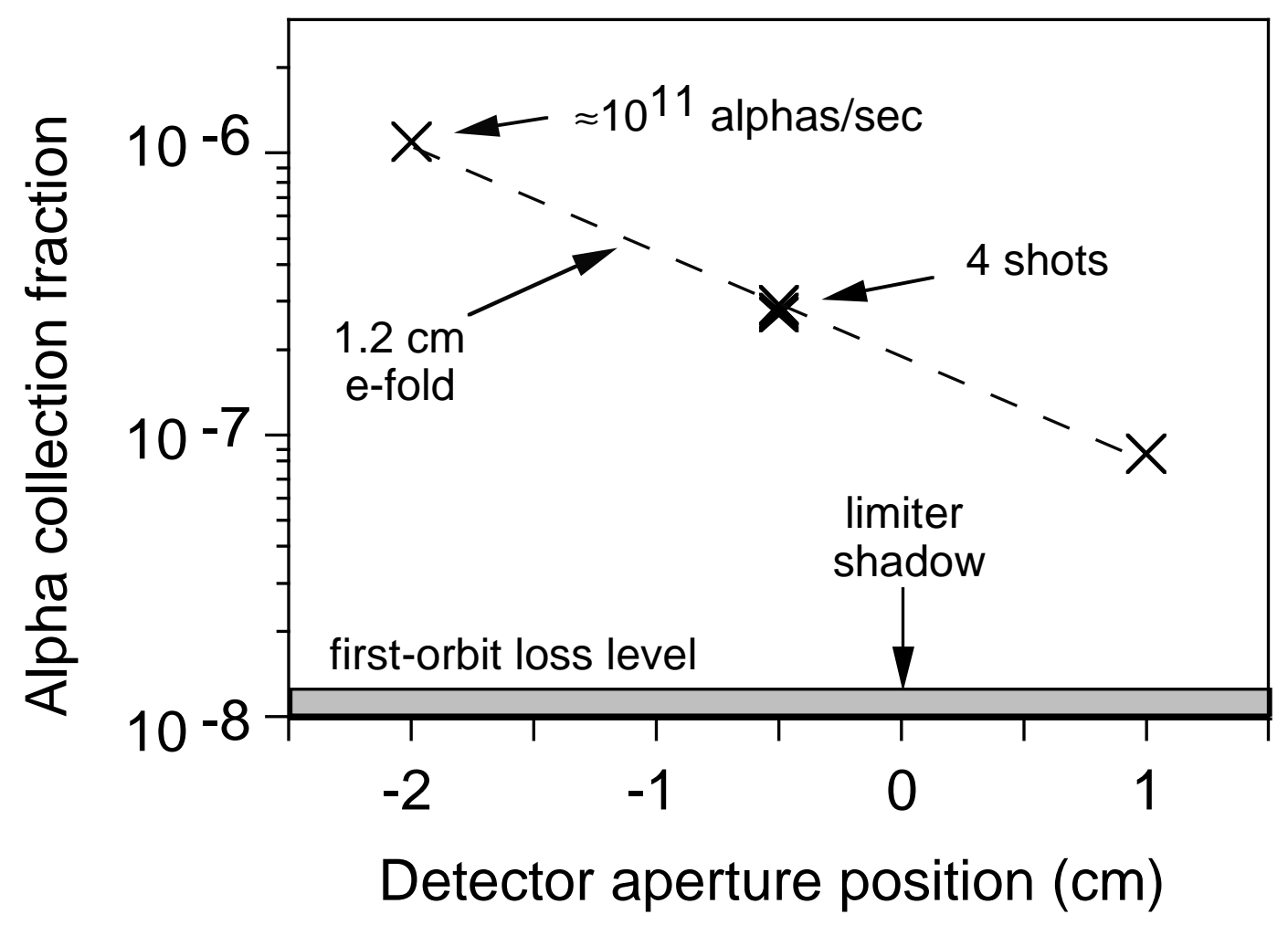

Figure 11 

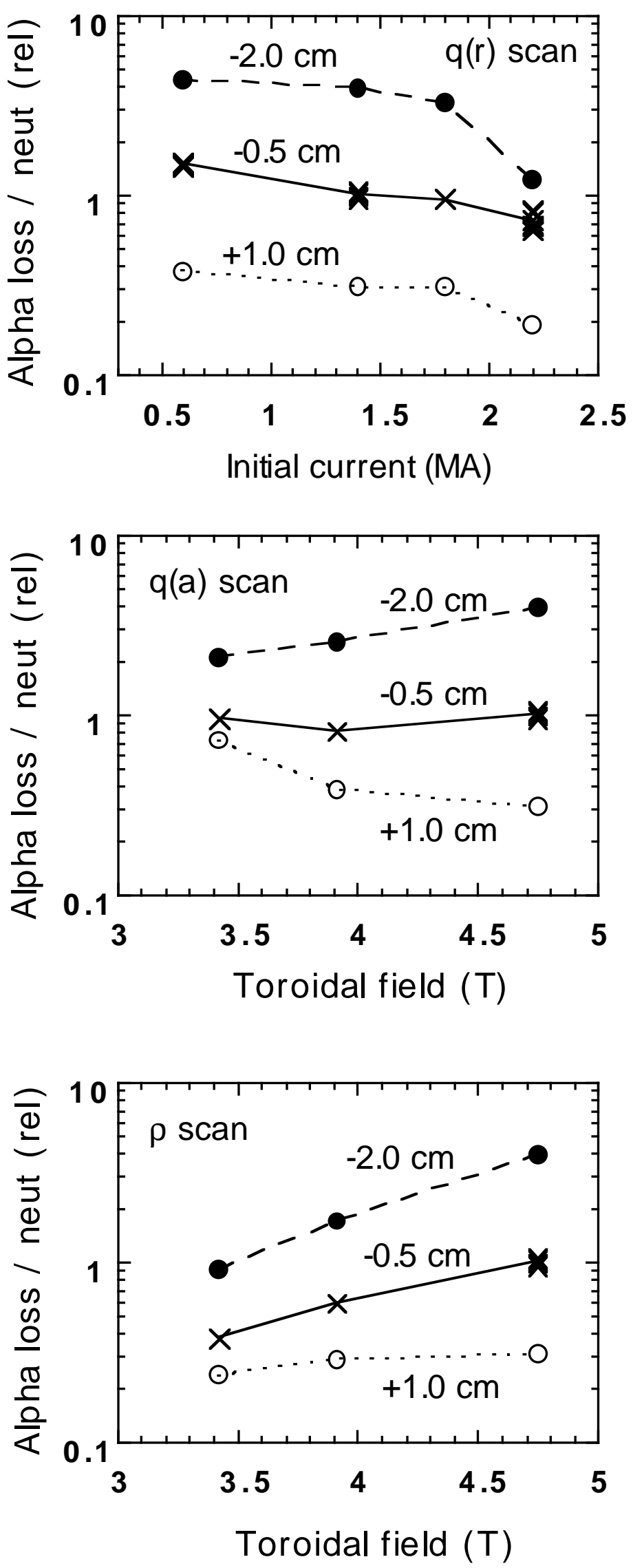

Figure 12 

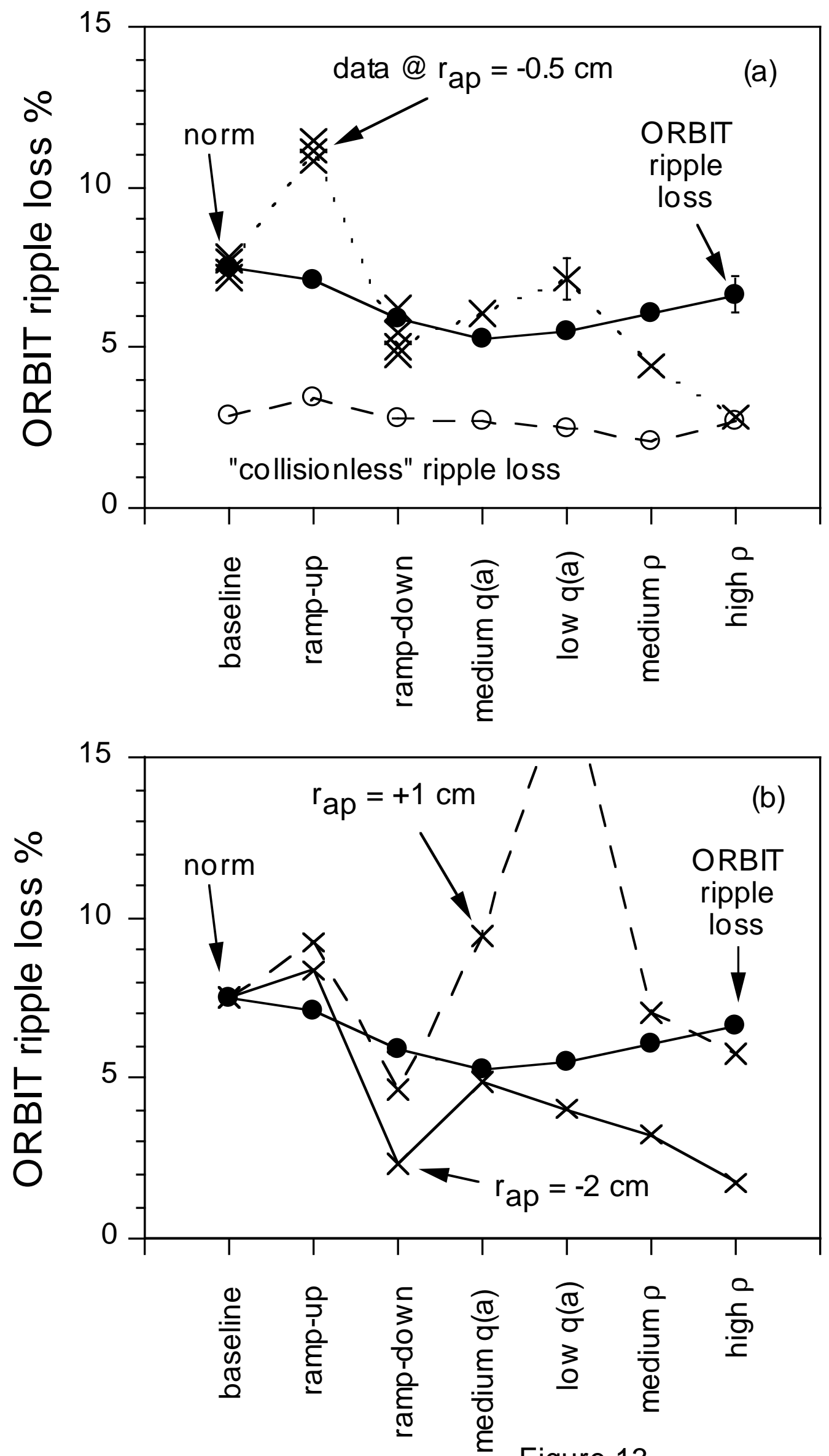

Figure 13 


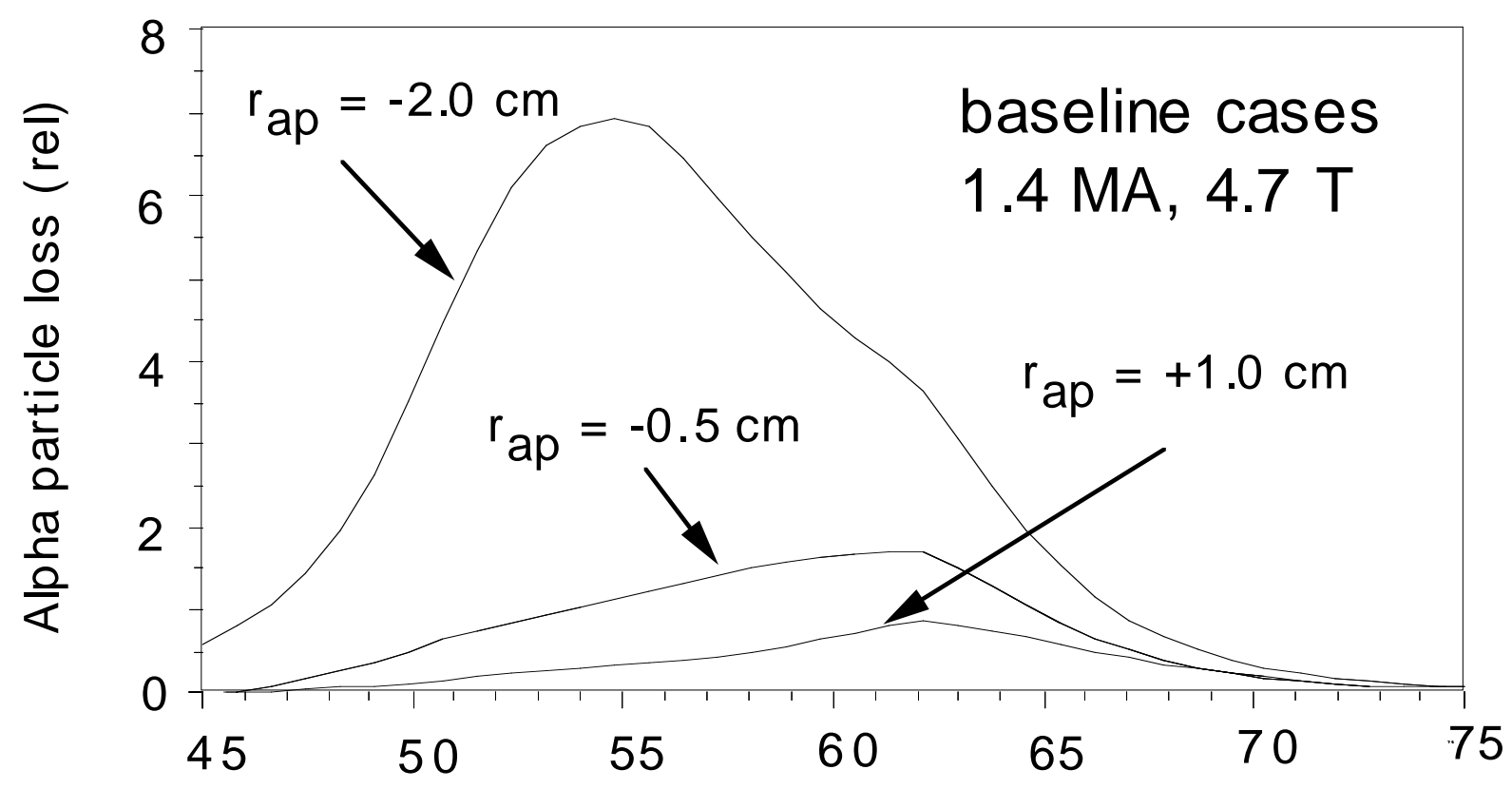

Toroidal pitch angle (deg)

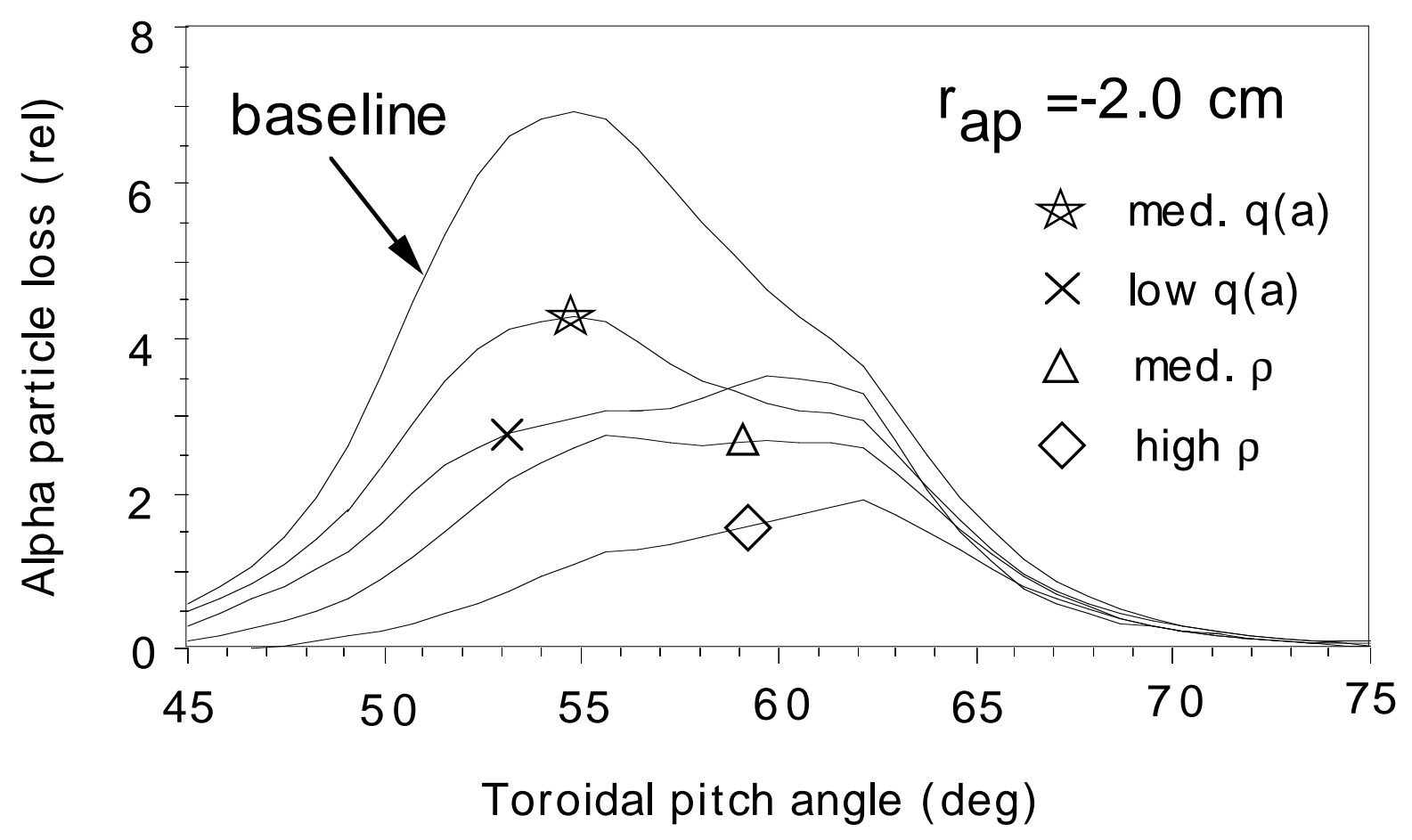

Figure 14 

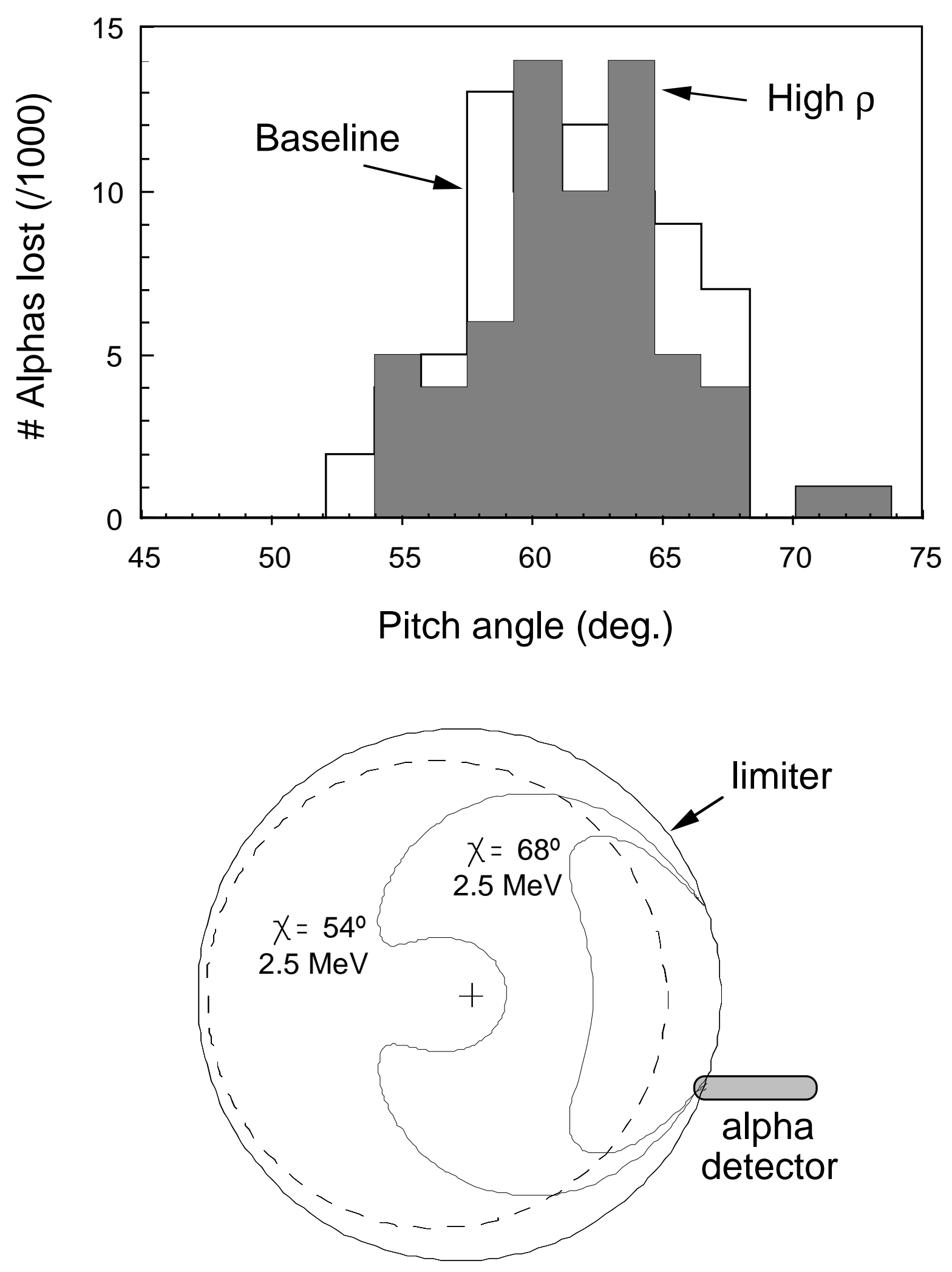

Figure 15 


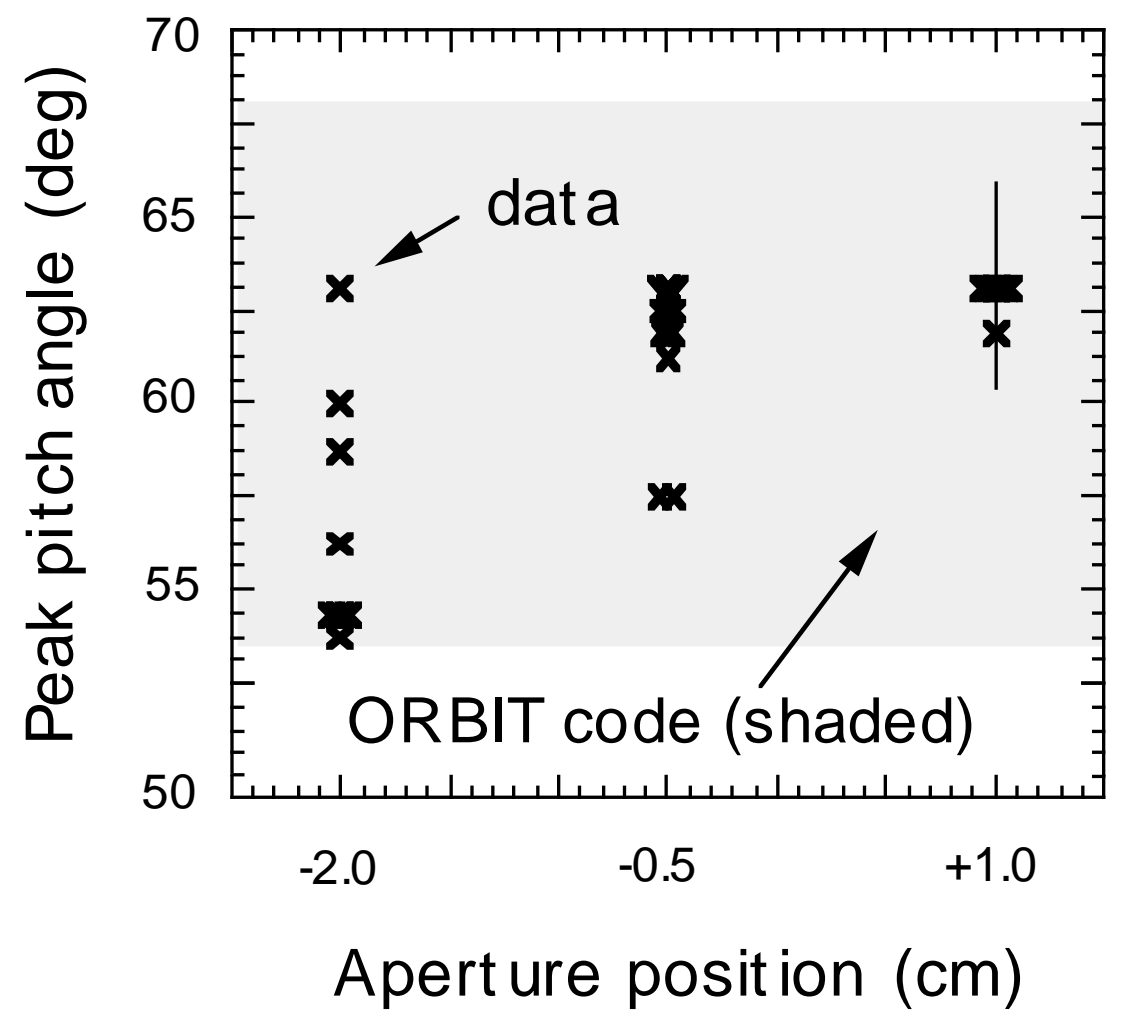

Figure 16 

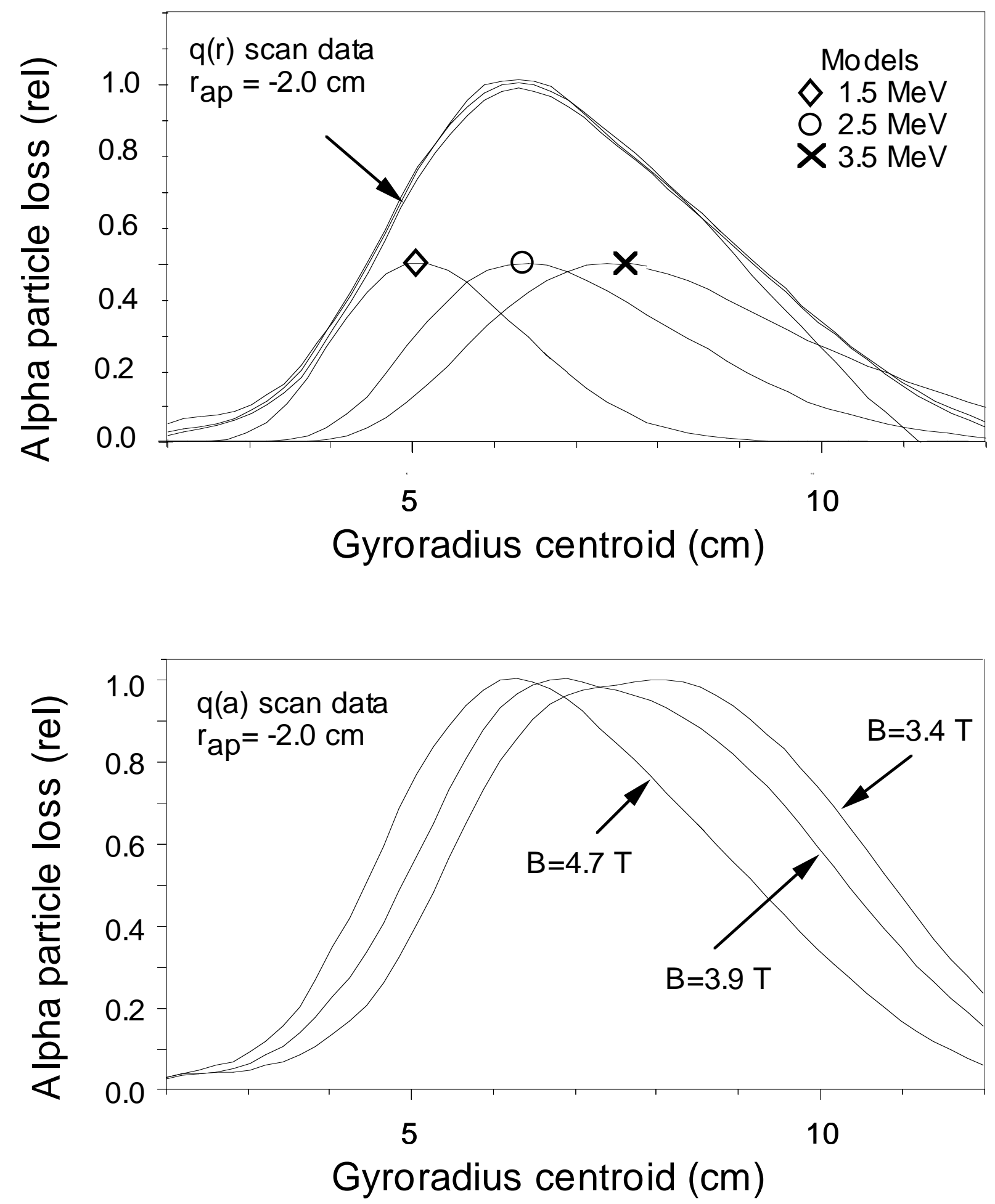

Figure 17 


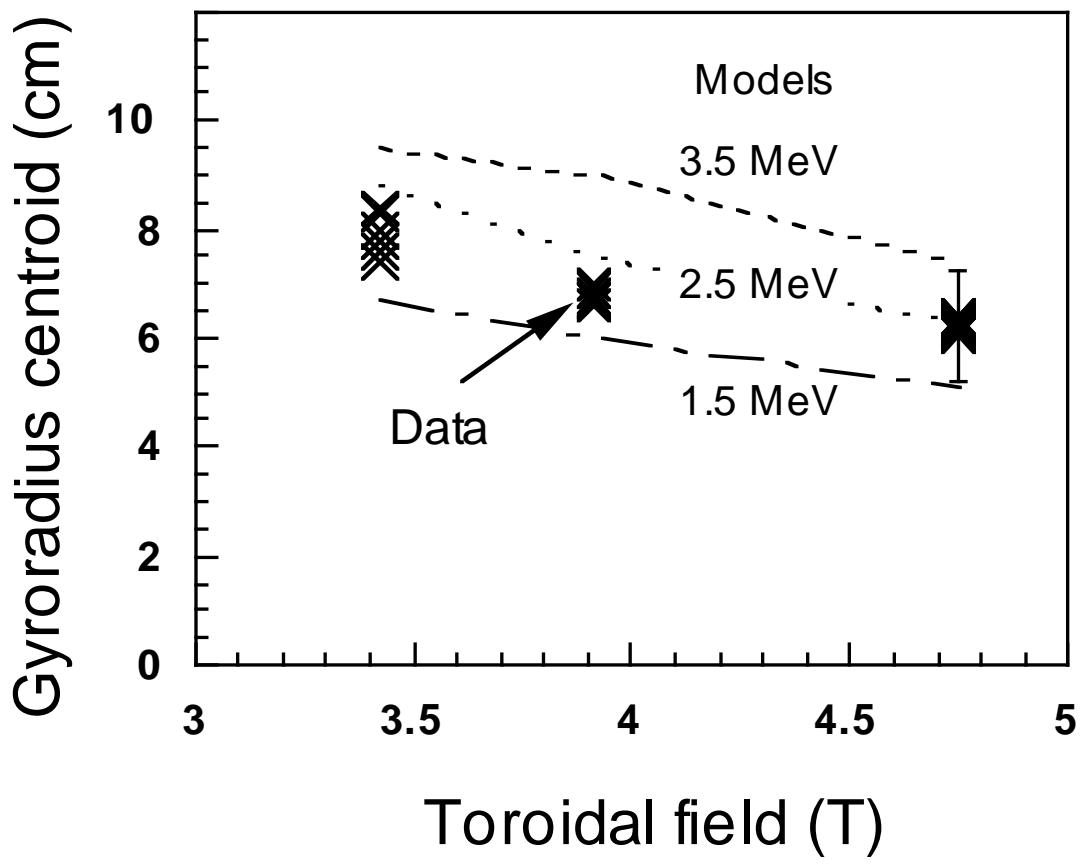

Figure 18 

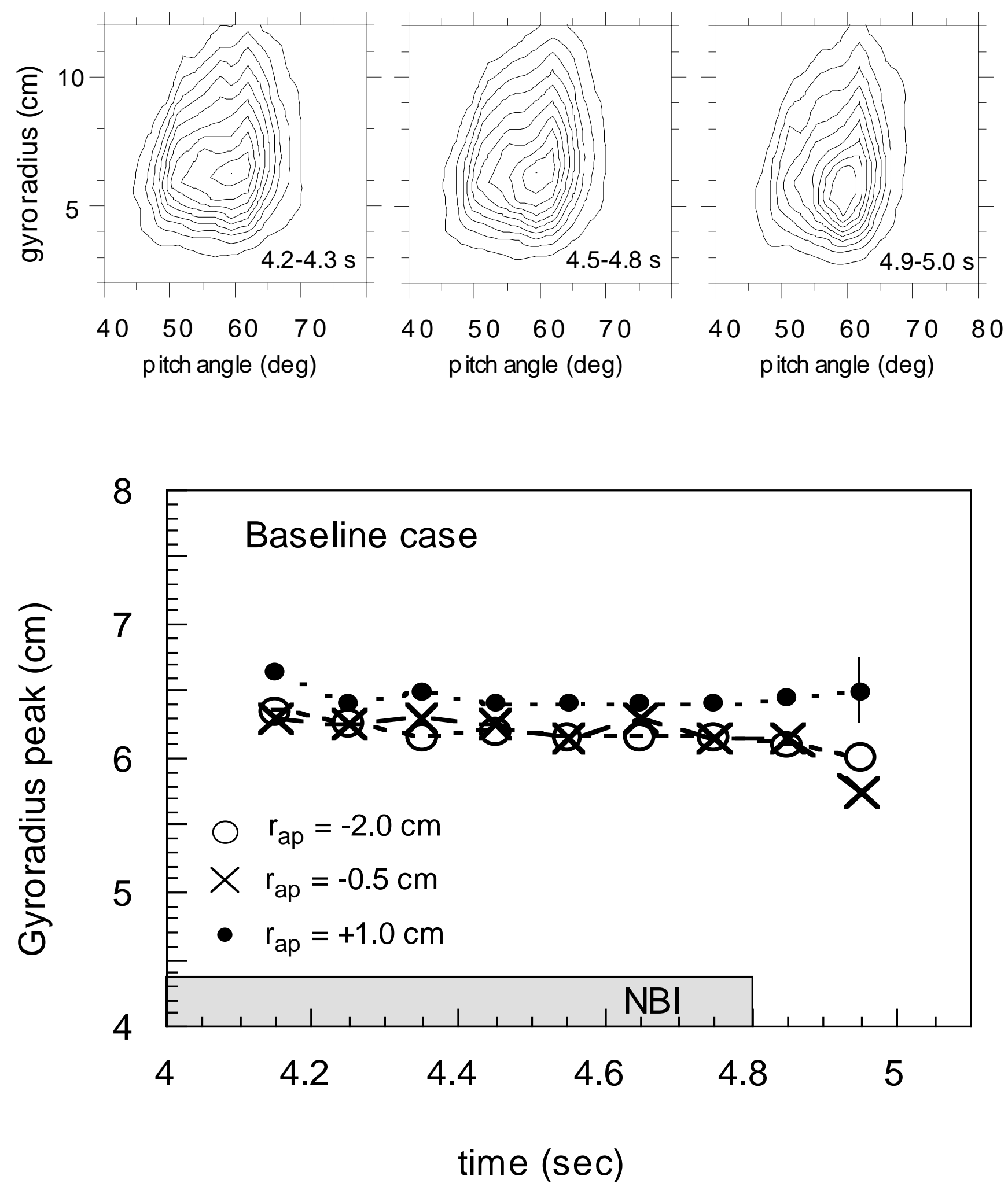

Figure 19 


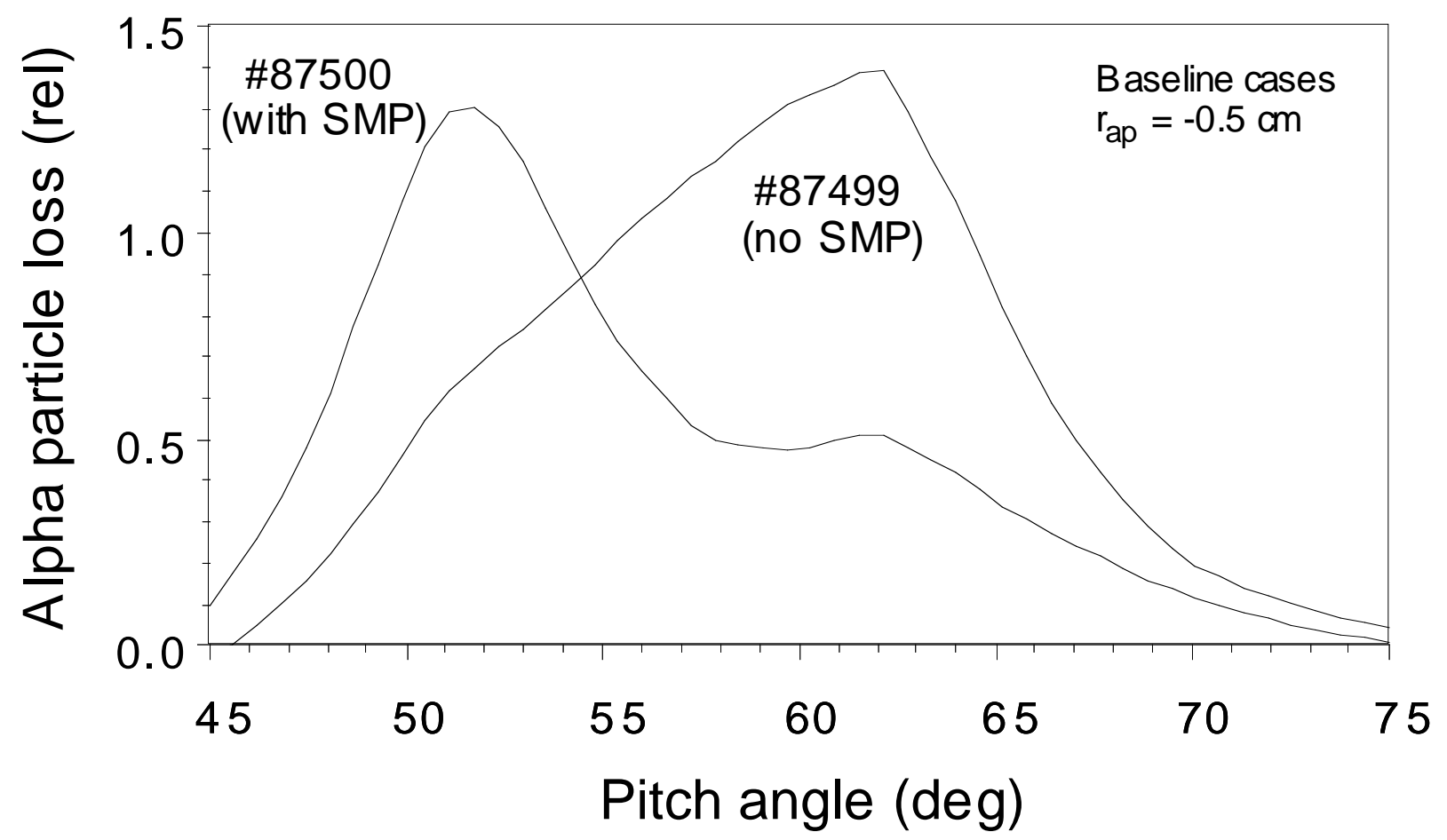

Figure 20 


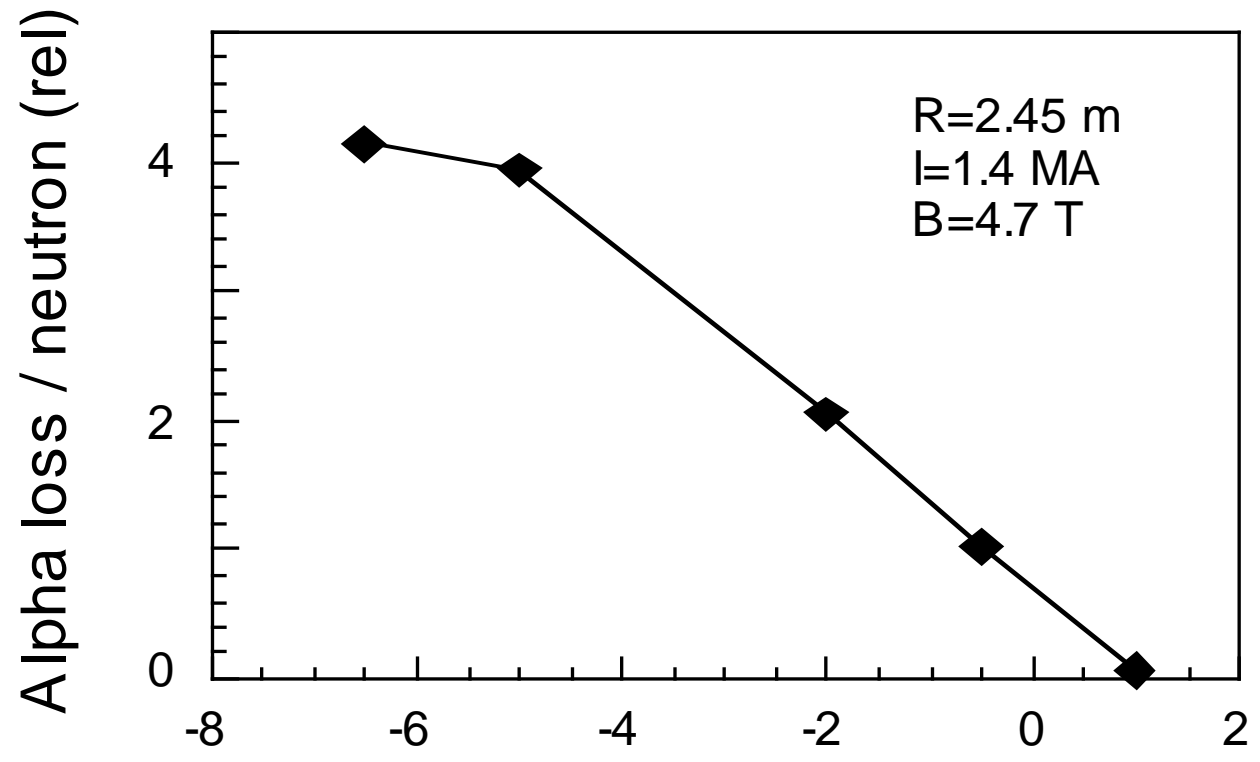

Probe aperture position $(\mathrm{cm})$

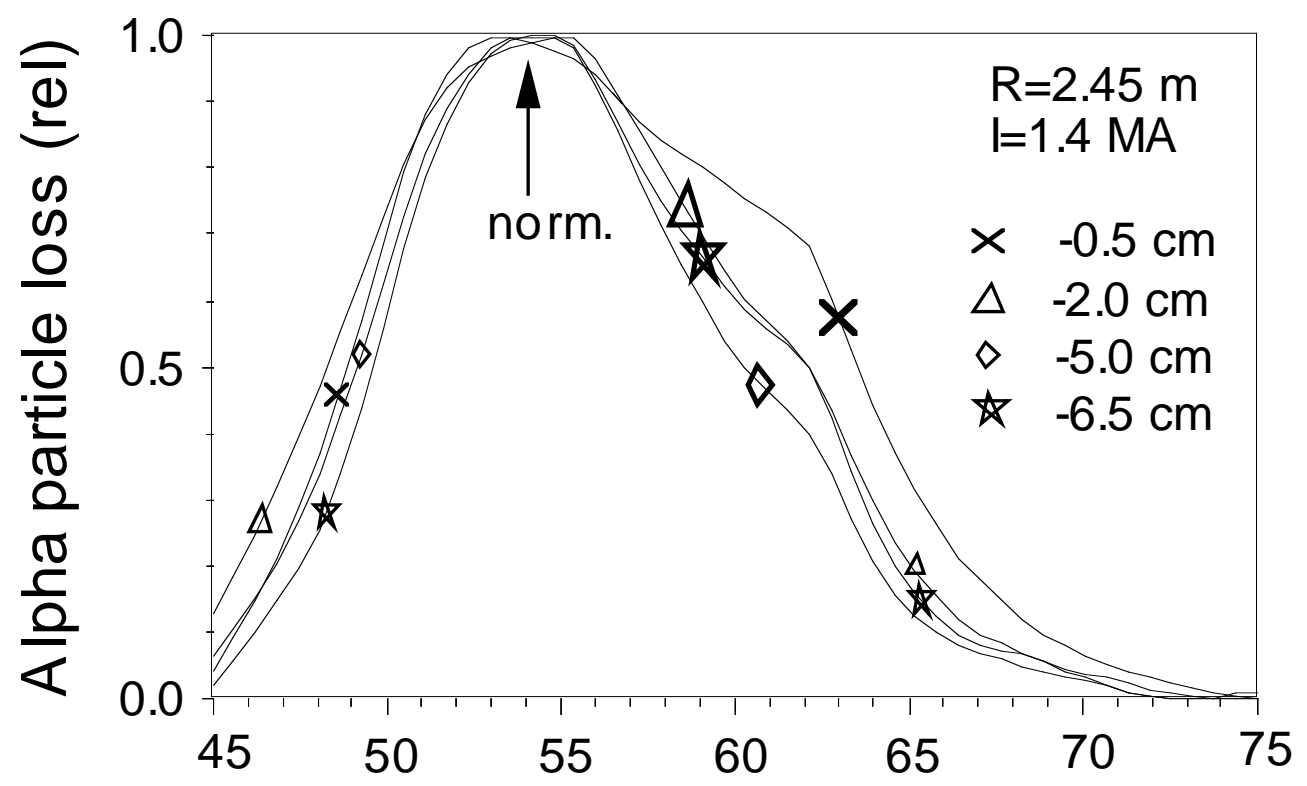

Pitch angle (deg)

Figure 21 

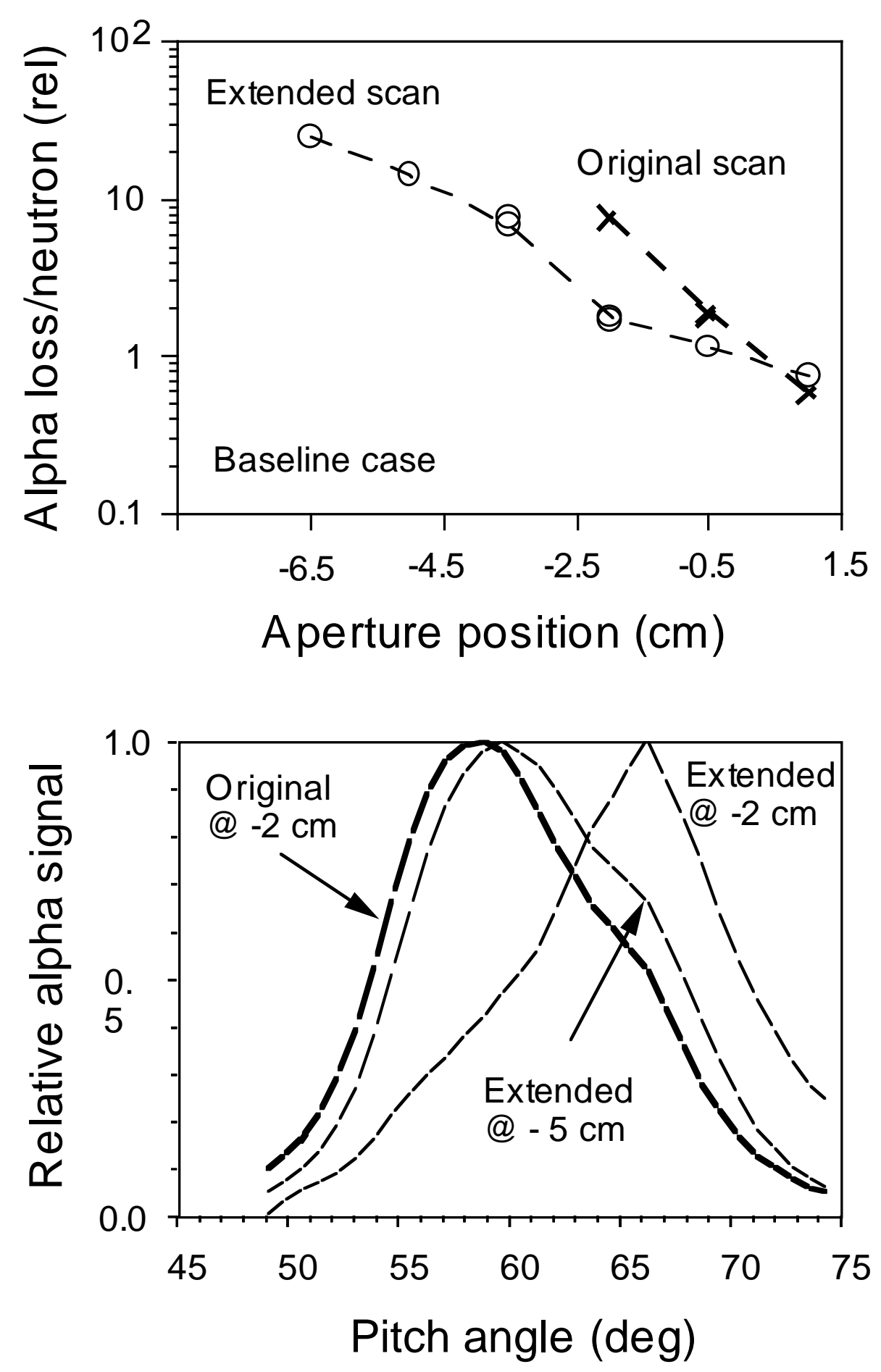

Figure 22 

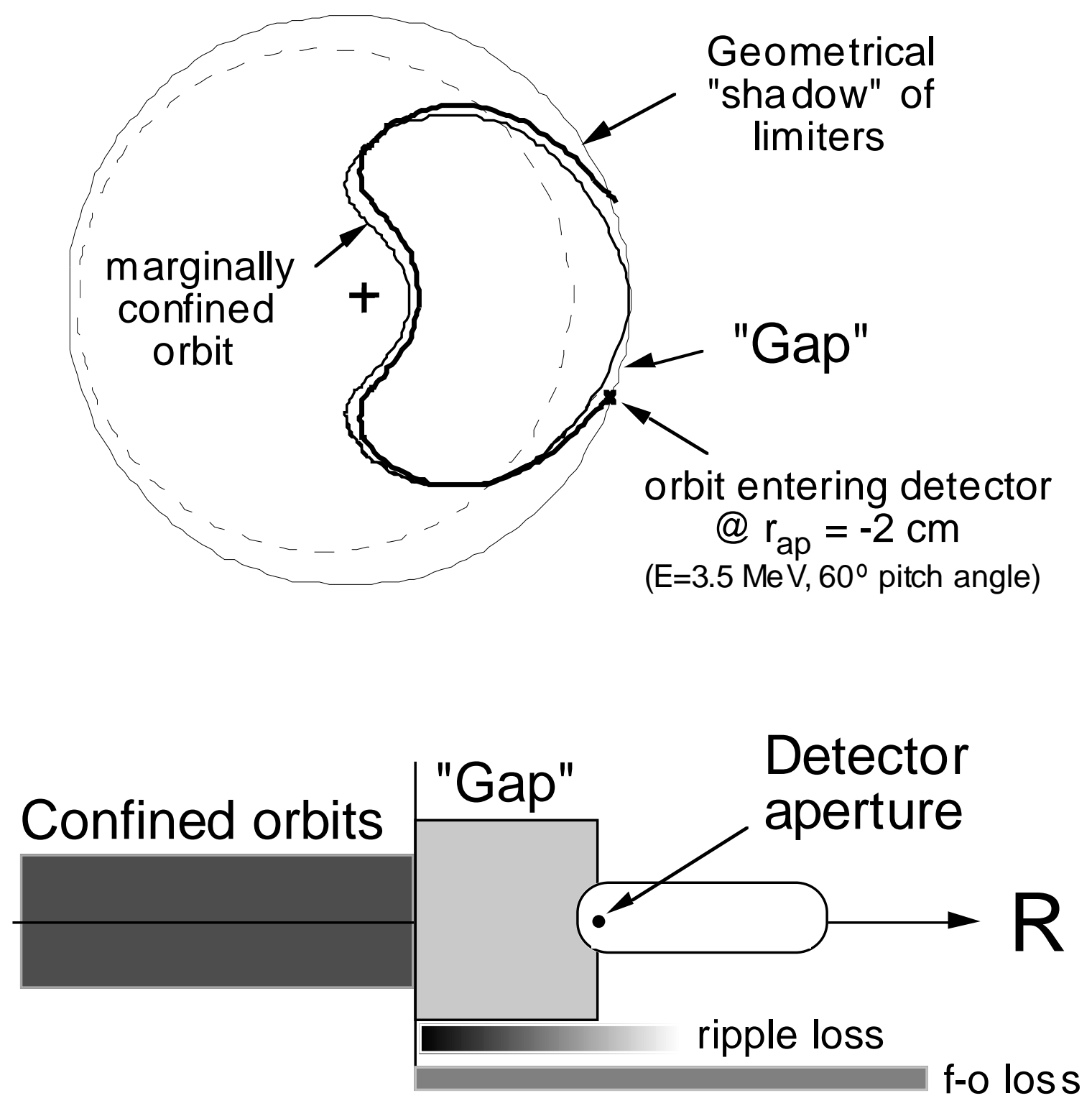

Figure 23 


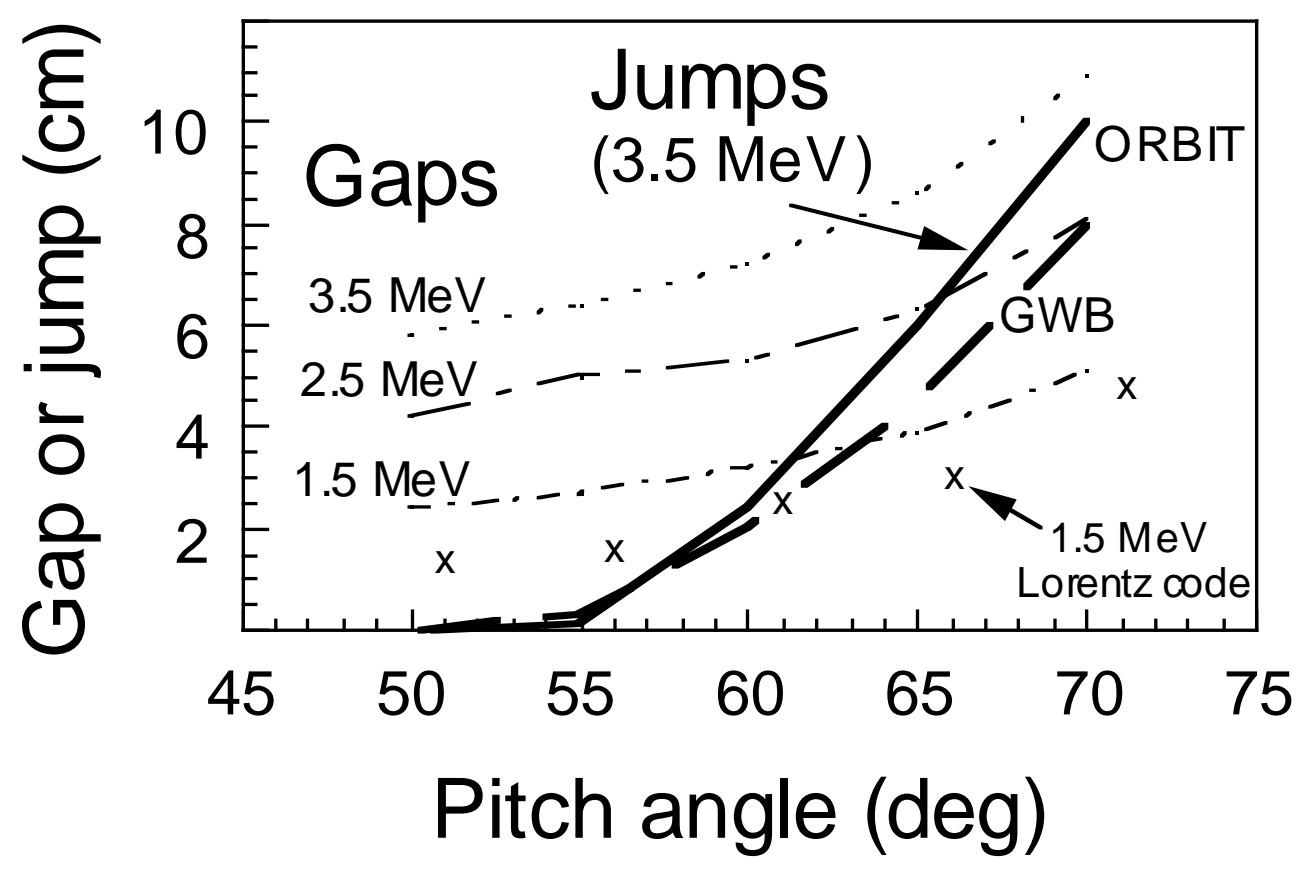

Figure 24 

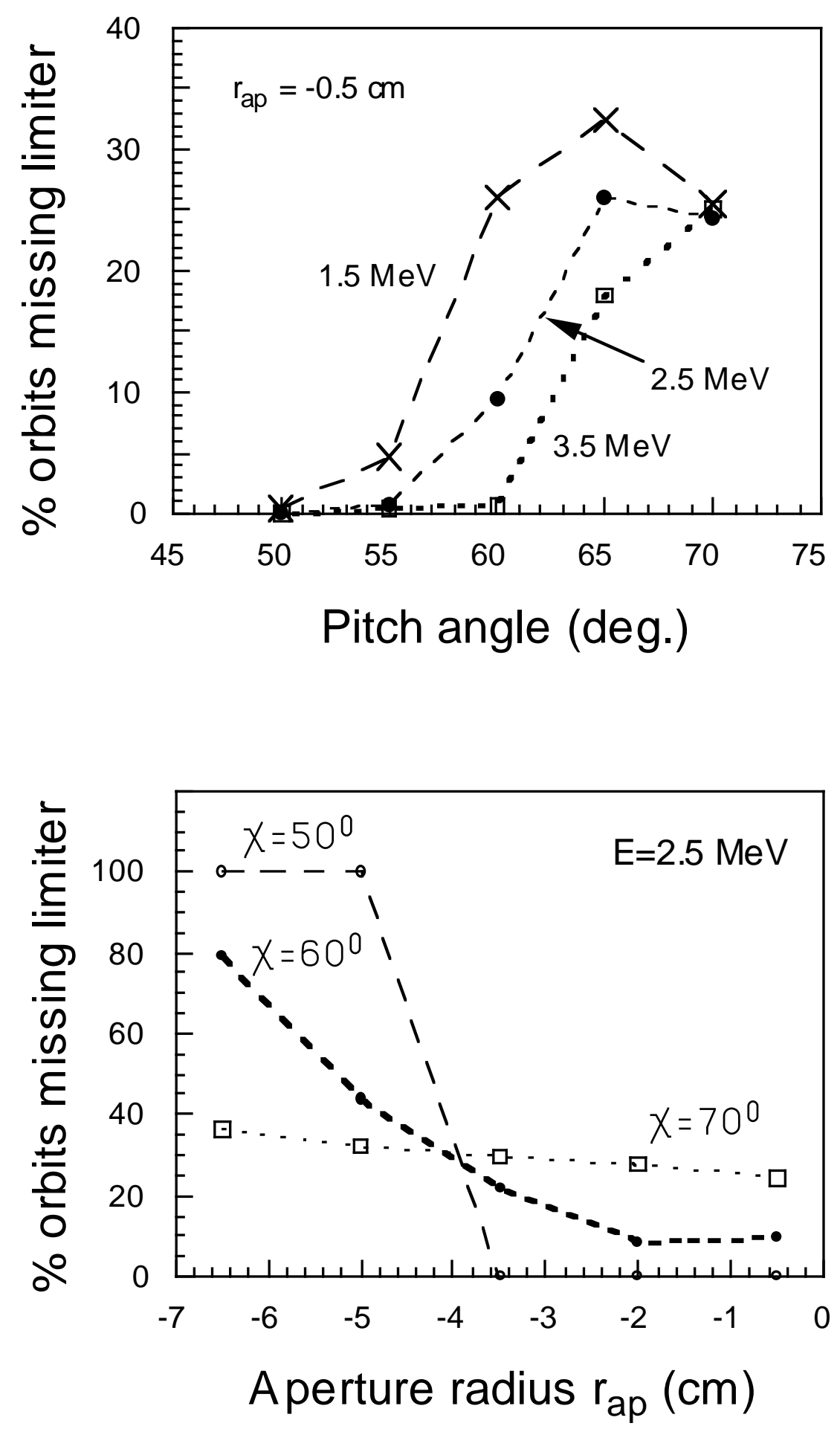

Figure 25 


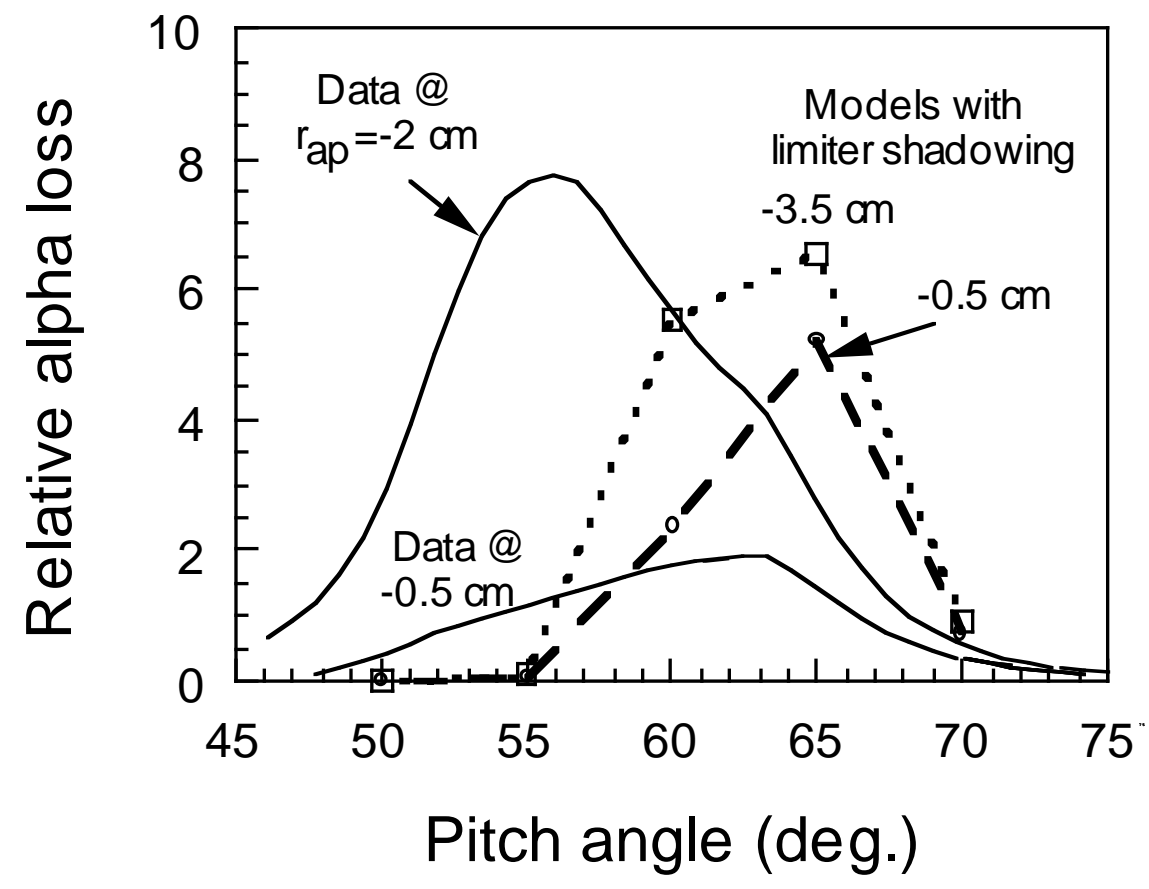

Figure 26 

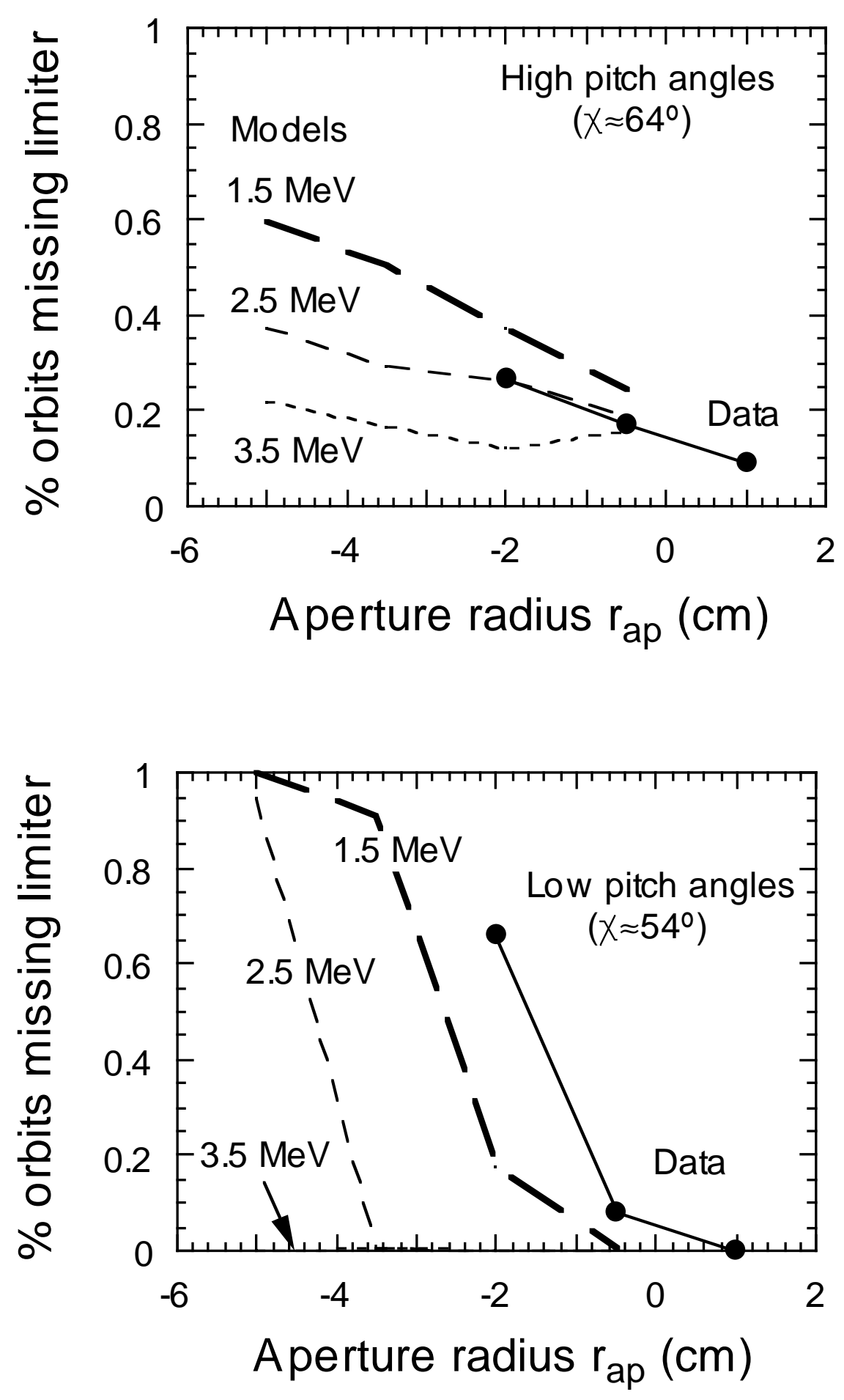

Figure 27 

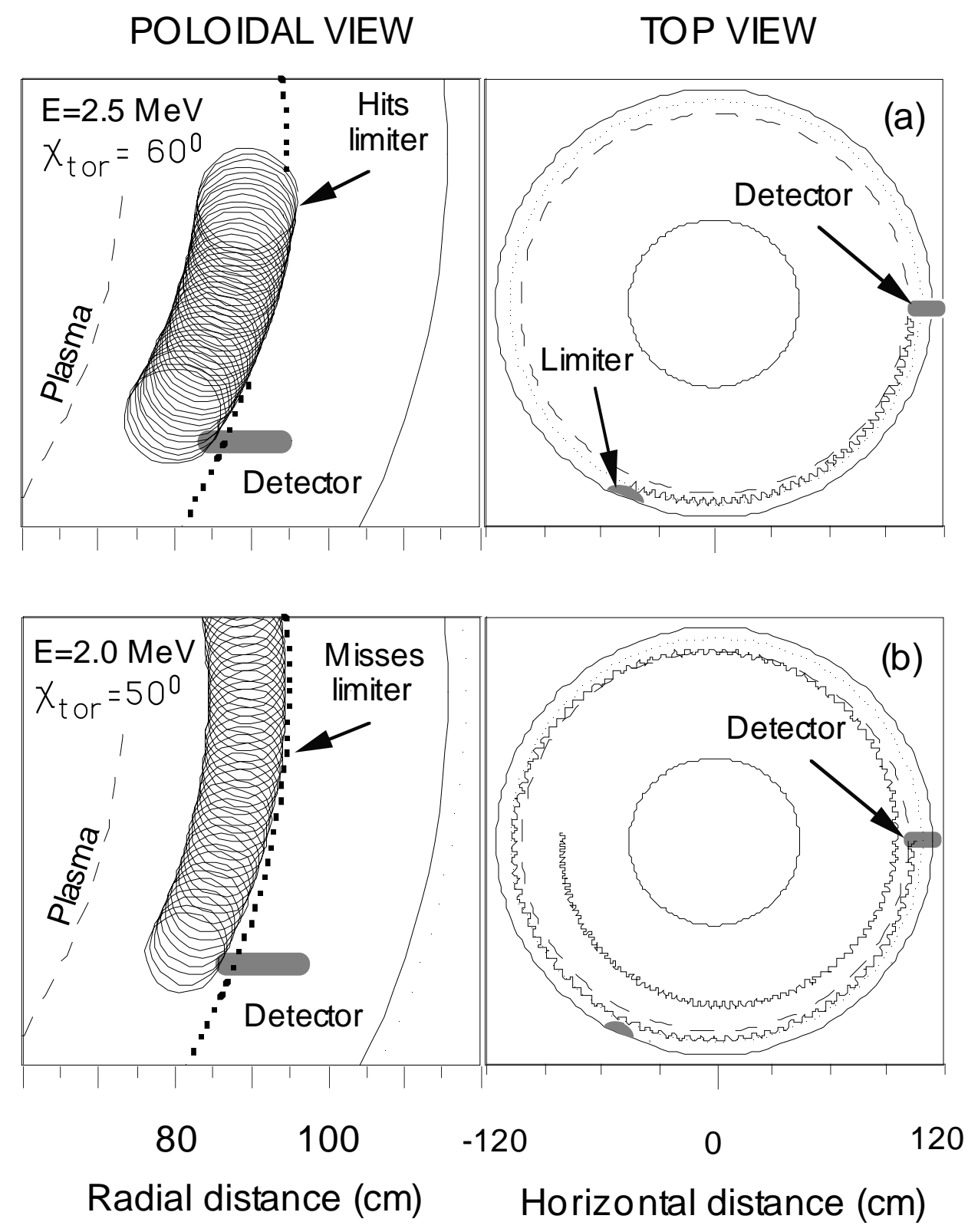

Figure 28 

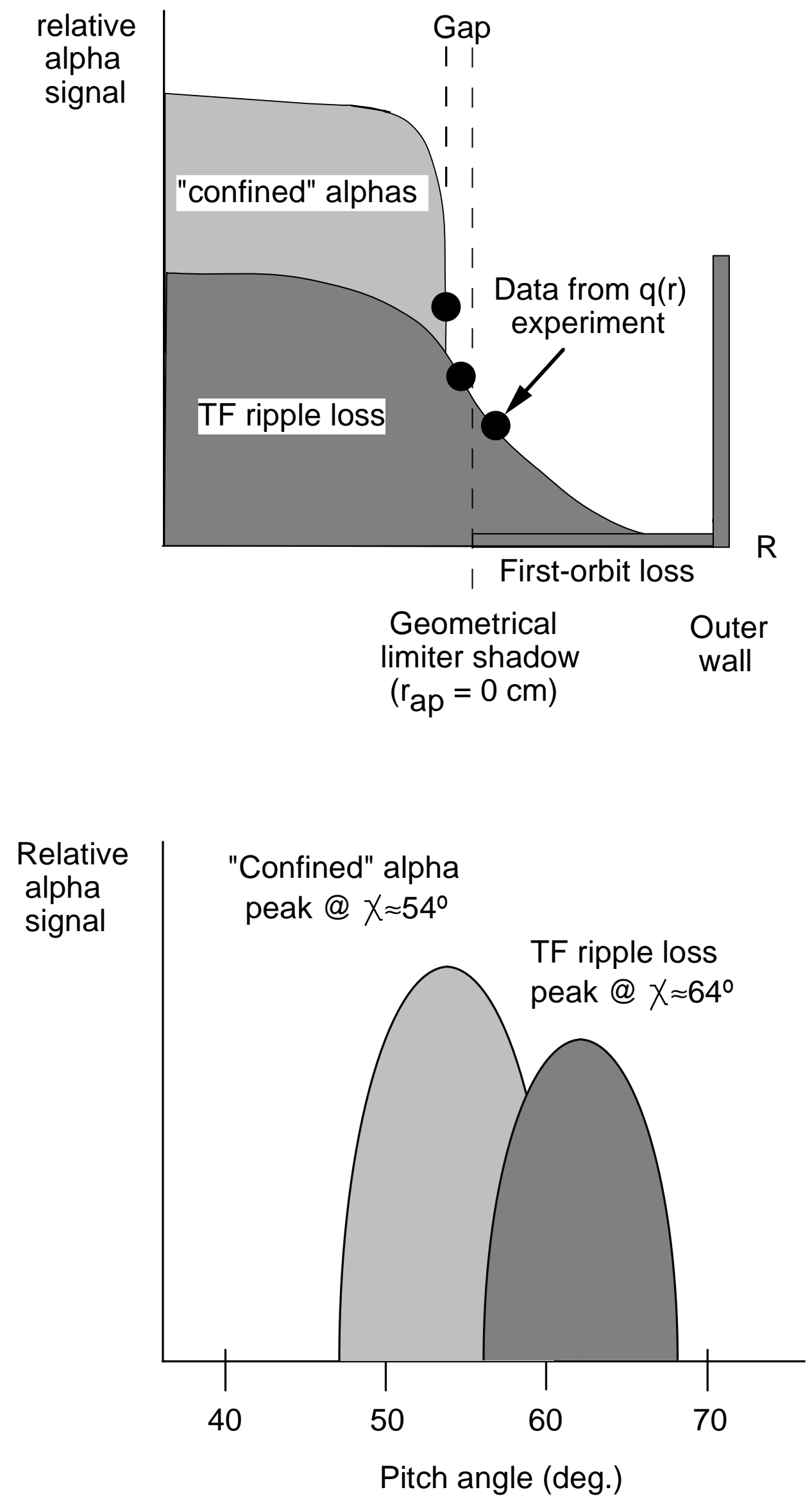

Figure 29 


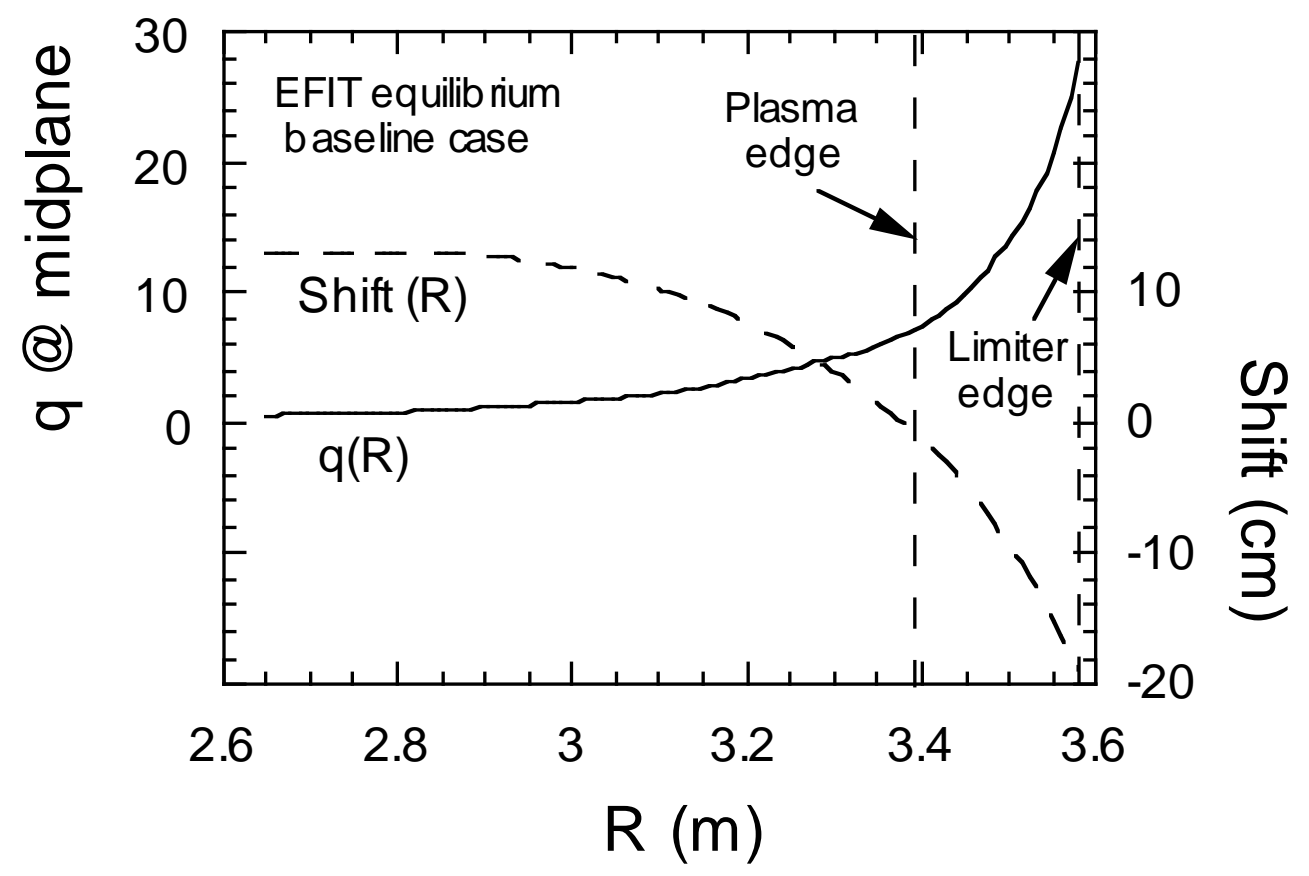

Figure 30 

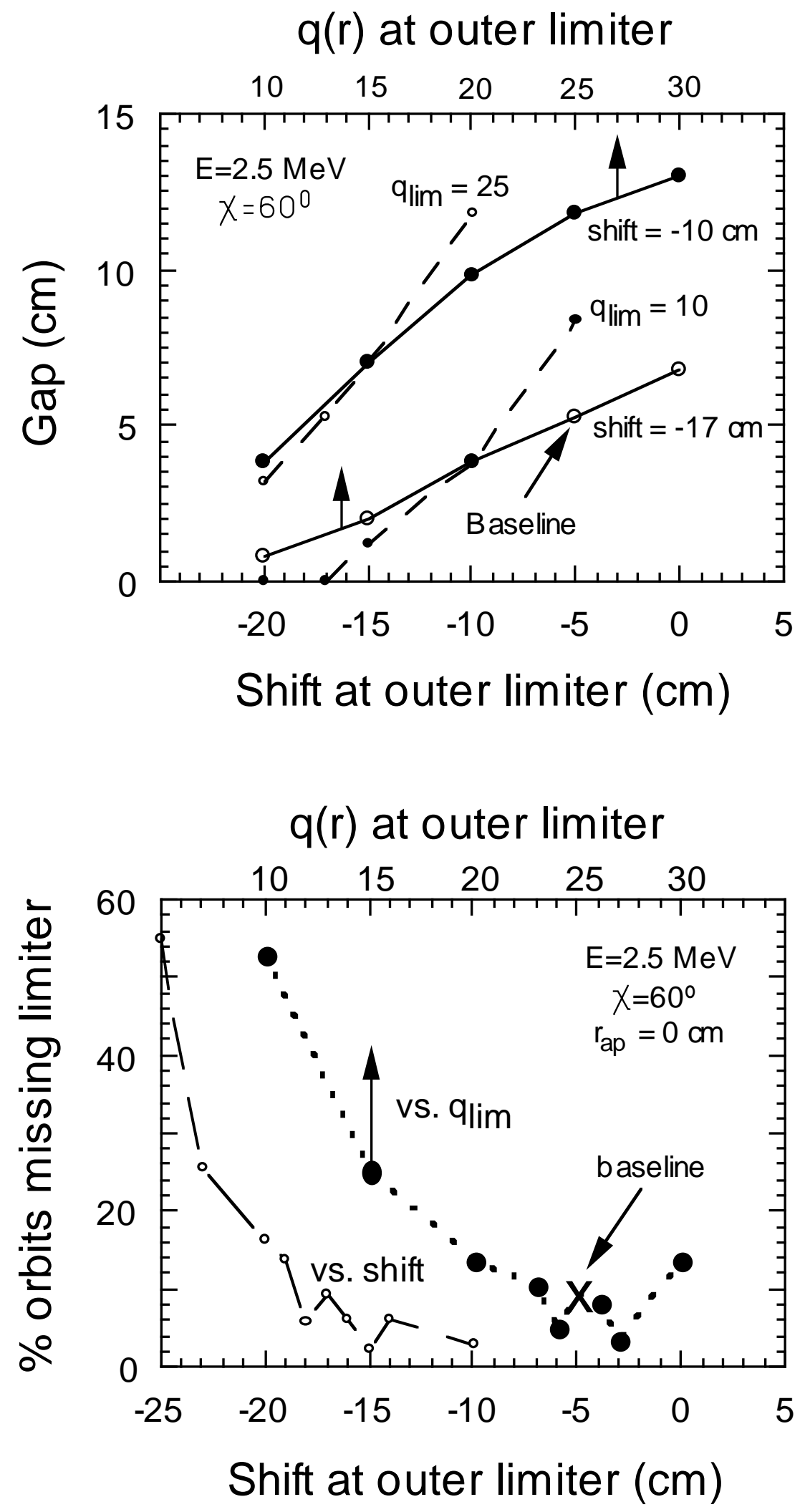

Figure 31 


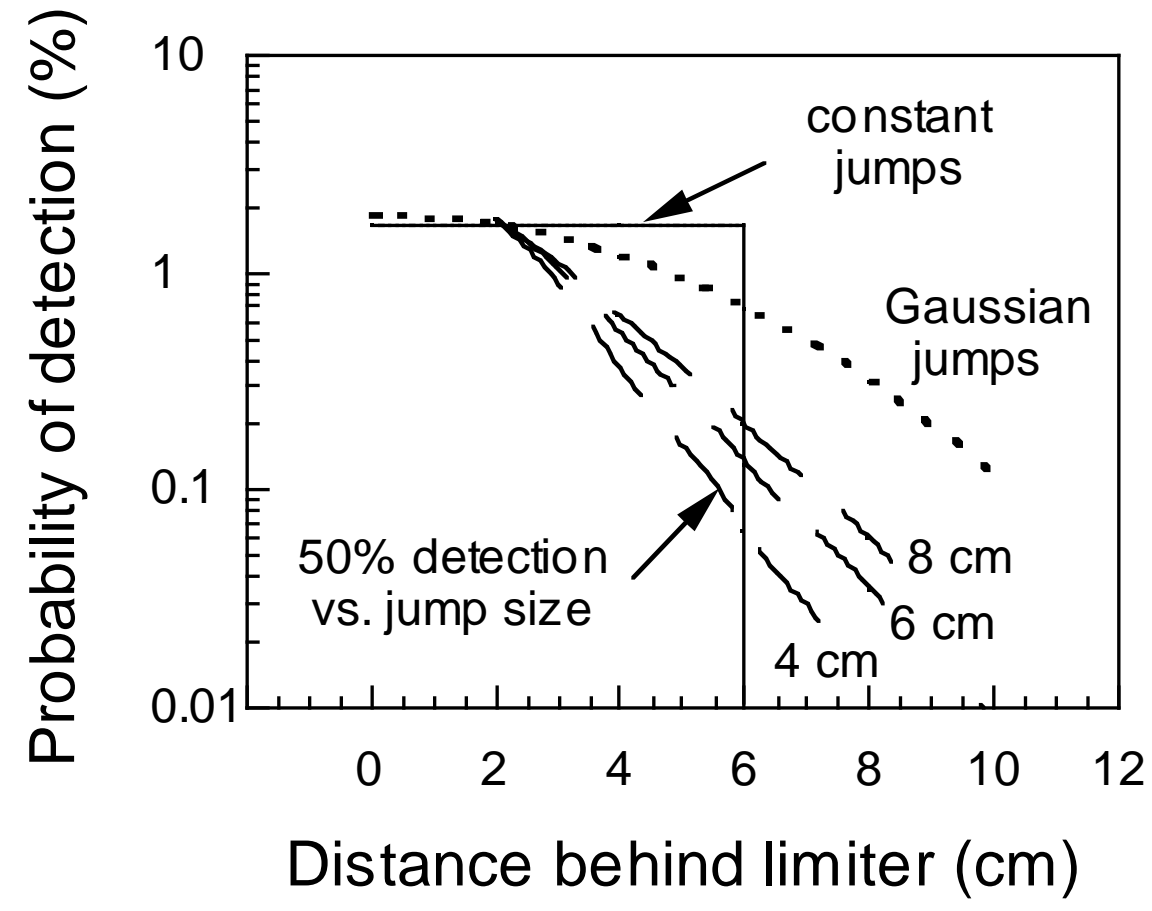

Figure 32 


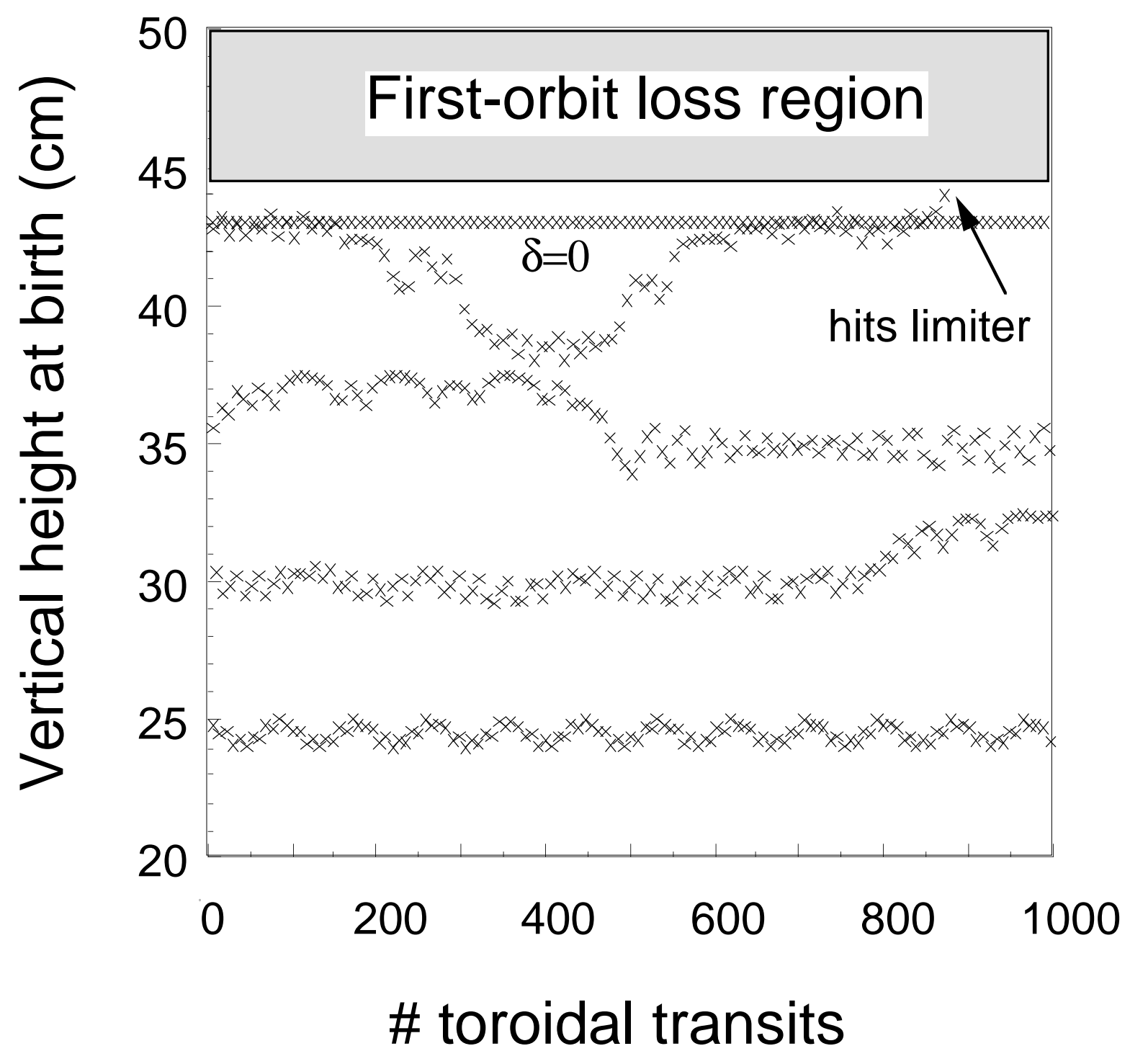

Figure 33 
